Indices 



\section{Index of Authors and Works}

For each author in Diels, this index lists the works for which manuscripts are recorded. It comprises two lists: one with the titles in Greek, and the other with the titles in Latin. In both lists, all the names of author and the titles are provided exactly as they appear in Diels. For the author names, some are in German (e.g., Hippokrates and Galenos) and others are in Latin. For the works, some are not identified by a specific title, but by a generic Latin designation (e.g., Opera varia, Excerpta varia, or Excerpta) or by a reference to an earlier publication. Furthermore, some works do not provide either a Greek title or a Latin translation. Whatever the form, items are listed in alphabetical order and followed by the reference to the part(s) and page number of Diels' lists where they appear, with the following abbreviations: "I" for the first part, devoted to Hippocrates and Galen (published in 1905), "II" for the second part, devoted to all the other physicians (published in 1906), and " $\mathrm{N}$ " for the supplement (N for Nachtrag = Supplement; published in 1908). A reference appearing as II.3, for example, indicates a work mentioned in part II, page 3 . 



\section{Greek titles}

\section{Abraham}

Excerpta varia: II.3 [= p. 201]; N.42 [= p. 383].

\section{Adamantius}

Excerpta varia: II.3 [= p. 201].

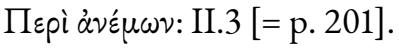

Пврі $\pi \alpha \lambda \mu \tilde{\omega} \nu:$ II.3 [= p. 201]; N.42 [= p. 383].

\section{Aelianus}

Epistula anepigrapha: II.4 [= p. 202].

\section{Aelius Promotus}

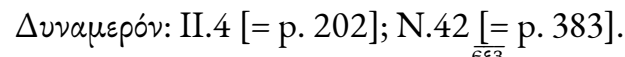

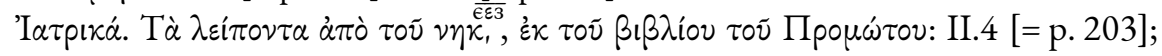

N.42 [= p. 383].

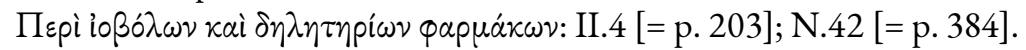

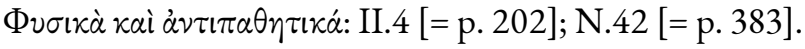

\section{Aëtius Amidenus}

Excerpta varia: II.7 [= p. 207]; N.43 [= p. 385].

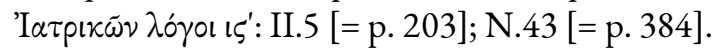

\section{Africanus}

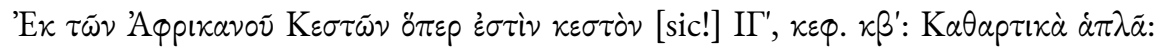

II.8 [= p. 209]; N.43 [= p. 385].

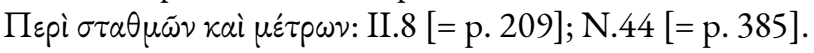

\section{Aglaias}

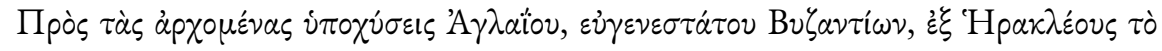

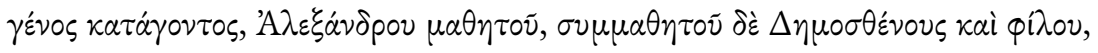

\section{Alexander}

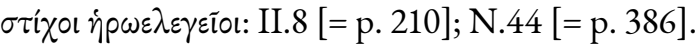

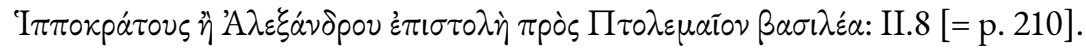

\section{Alexander Aphrodisiensis}

Excerpta varia: II.10 [= p. 213].

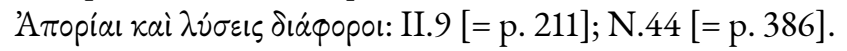

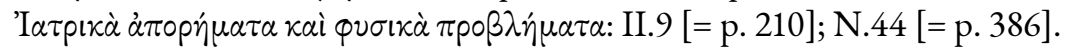

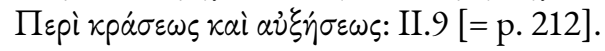

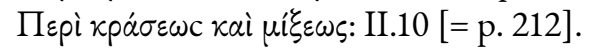

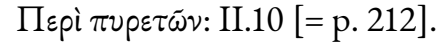

\section{Alexander Rex}

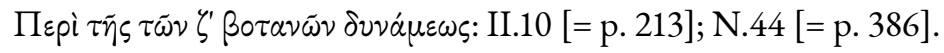




\section{Alexander Sophista}

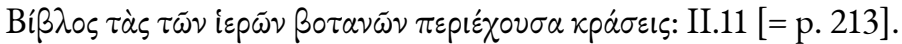

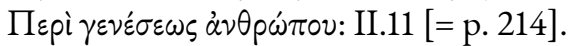

\section{Alexander Trallianus}

Excerpta varia: II.13 [= p. 216].

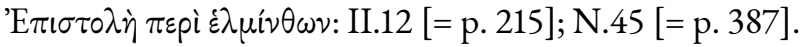

$\Theta \varepsilon p a \pi \varepsilon v \tau \iota x \alpha ́:$ II.11 [= p. 214].

Пврі̀ ò $\varphi \Delta \lambda \mu \omega \tilde{\omega} v:$ II.12 [= p. 215].

Alexander (Trallianus?)

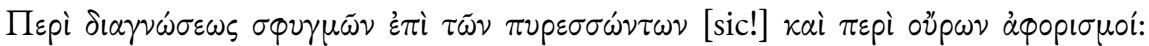

II.13 [= p. 216].

\section{Andreas Caesareae in Cappadocia Archiepiscop[us]}

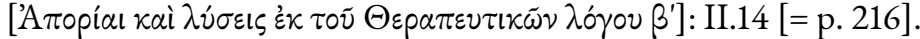

\section{Andreiomenus}

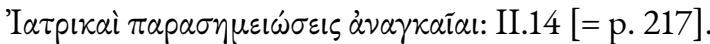

\section{Andromachus}

Excerpta: II.14 [= p. 217].

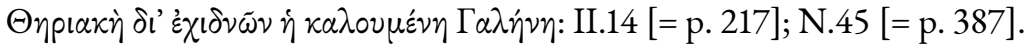

Antonius Pyropulus

Excerpta varia: II.15 [= p. 217]; N.45 [= p. 387].

\section{Antyllus}

Excerpta varia: II.15 [= p. 218].

\section{Apollonius Citiensis}

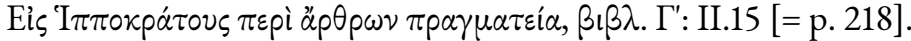

\section{Archelaus}

Fragmentum: II.16 [= p. 219].

\section{Archigenes}

\section{Aretaeus}

Excerpta varia: II.16 [= p. 219].

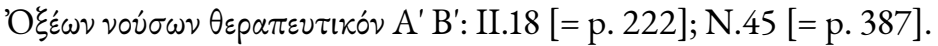

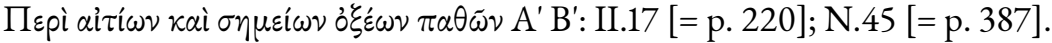

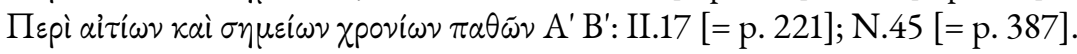

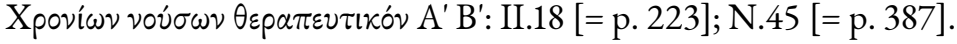

\section{Artemidorus}

Пвpì ov̉p $\omega \nu$ : II.19 [= p. 224].

\section{Asclepiades}

Excerpta varia: II.20 [= p. 225].

$\Upsilon \gamma \mid \varepsilon เ \nu \grave{\alpha} \pi \alpha p \alpha \gamma \gamma \dot{\varepsilon} \lambda \mu \alpha \tau \alpha:$ II.20 [= p. 225]; N.46 [= p. 388].

\section{Athenaeus}

Excerpta: II.21 [= p. 227]. 


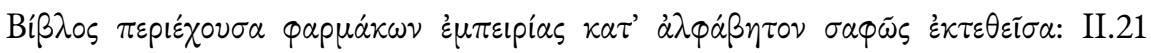
$[=$ p. 226].

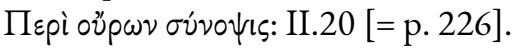

\section{Basilius (S[anctus])}

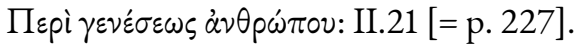

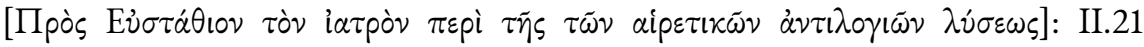
[= p. 227]; N.46 [= p. 388].

\section{Beniaminus}

Excerpta: II.22 [= p. 228]; N.46 [= p. 388].

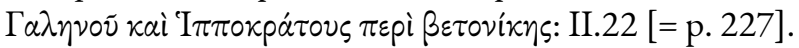

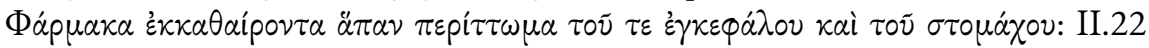
[= p. 228].

\section{Cassius Iatrosophista}

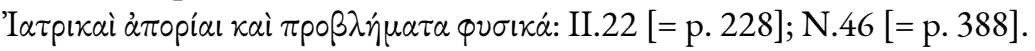

\section{Cornelius Celsus}

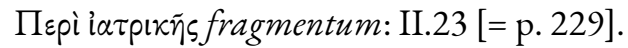

\section{Chariton}

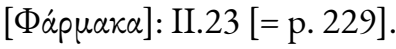

Chrysostomius (?)/Chrysostomus (sic!) (see Ioannes Chrysostomus = II.52 [= p. 266] and N.46 [= p. 389]).

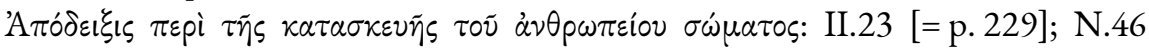

\section{Cleopatra}

$$
\text { [= p. 389]. }
$$

Excerpta varia: II.24 [= p. 230].

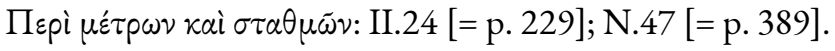

\section{Coeranus}

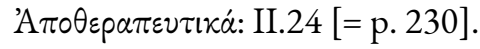

\section{Constantinus Melitiniota}

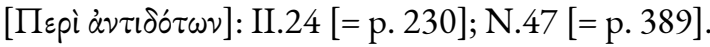

Constantinus Taronites (see N.67 [= p. 418]: Taronitus [?])

Excerpta: N. 47 [= p. 389].

\section{Crateuas}

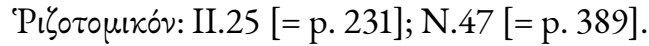

\section{Craton}

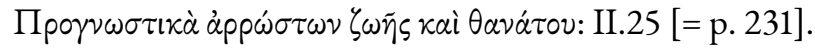

\section{Cyrillus Alexandrinus}

Excerpta: II.25 [= p. 231].

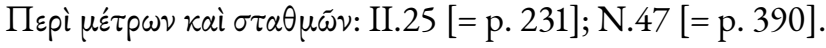

\section{Damascenus}

Excerpta: II.25 [= p. 232]. 


\section{Damascius}

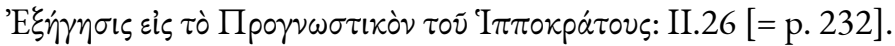

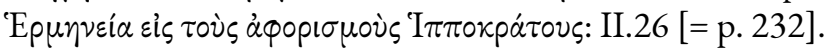

\section{Damnastes}

Excerpta: II.26 [= p. 233].

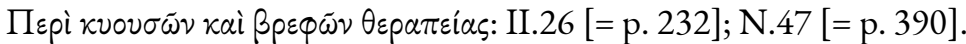

\section{Democritus}

Excerpta varia: II.27 [= p. 233]; N.48 [= p. 390].

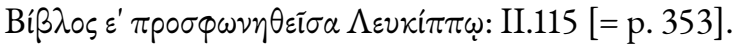

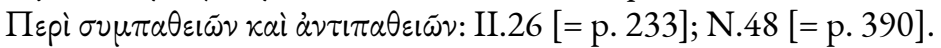

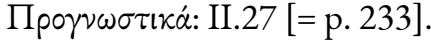

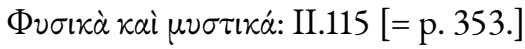

\section{Diocles}

Excerpta varia: II.29 [= p. 235].

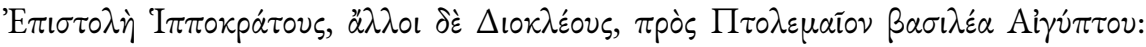

II.28 [= p. 235].

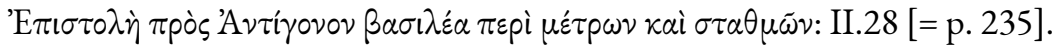

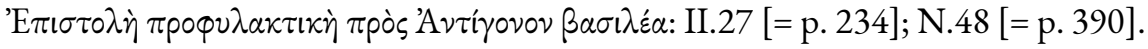

\section{Diodorus}

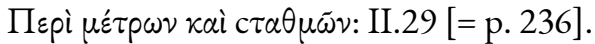

\section{Dionysius Simplex}

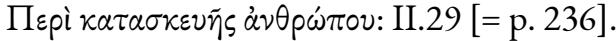

\section{Dioscurides}

Excerpta/Excerpta varia: II.34 [= p. 243]; N.49 [= p. 393].

Opera varia: II.29 [= p. 236]; N.48 [= p. 391].

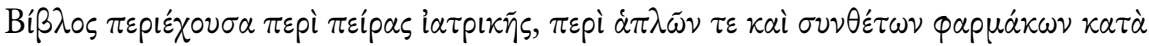

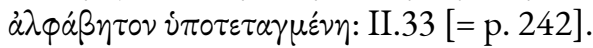

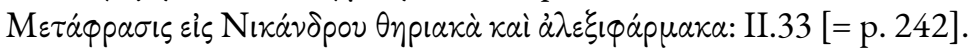

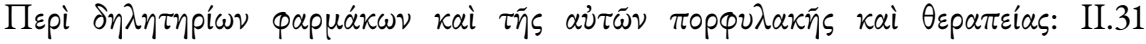
[= p. 239]; N.49 [= p. 391].

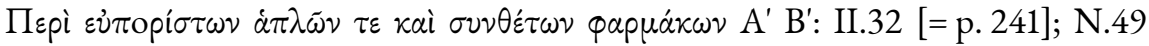
[= p. 392].

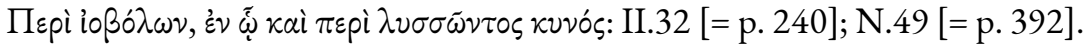

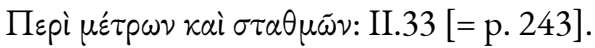

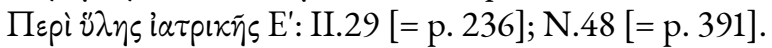

\section{Eleutherus}

Excerpta: II.35 [= p. 245].

\section{Epiphanius}

Excerpta varia: II.36 [= p. 247].

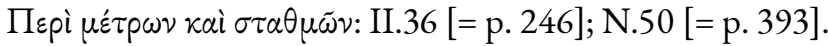




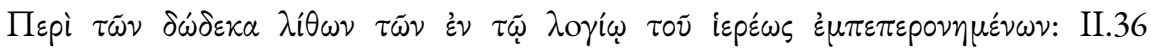
[= p. 247]; N. 50 [= p. 394].

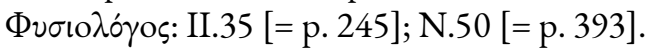

\section{Erasistratus}

Excerpta varia: II.36 [= p. 247].

\section{Erotianus}

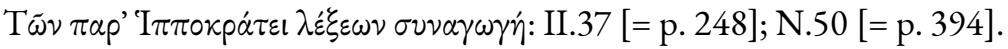

\section{Esdras Propheta}

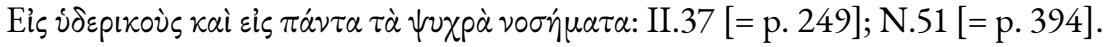

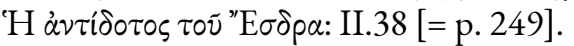

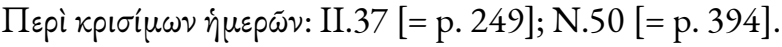

\section{Euphemius Siculus}

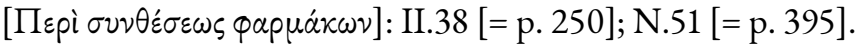

\section{Eusebius}

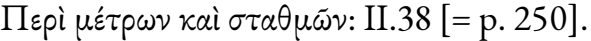

\section{Eustathius}

\section{Eutecnius}

Excerpta: II.38 [= p. 250].

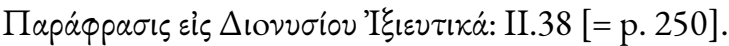

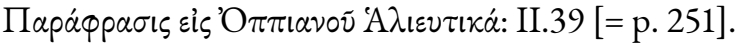

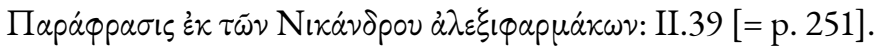

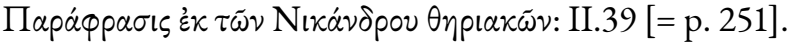

\section{Galenos}

Excerpta varia: I.148 [= p. 192]; N.41 [= p. 380].

Indices in Galenum: I.150 [= p. 194].

Opera varia: I.58 [= p. 93]; N.29 [= p. 367].

Scholia in Galenum: I.150 [= p. 194].

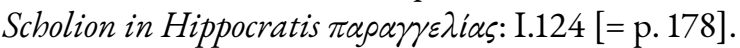

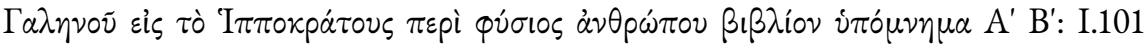

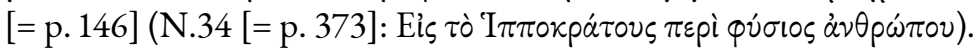

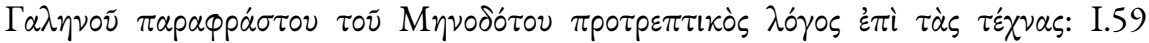
[= p. 93].

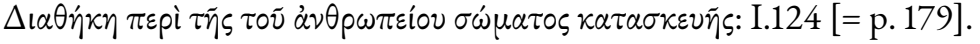

$\Delta$ ıаip $\sigma \iota s:$ I.118 [= p. 171].

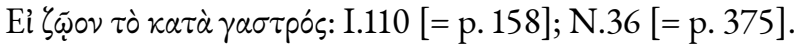

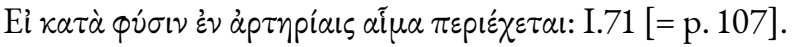

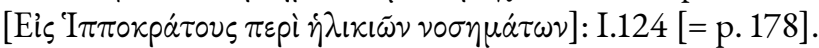

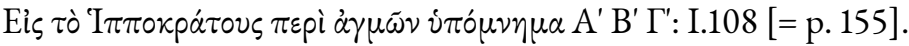

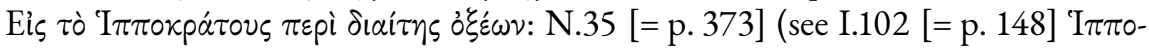

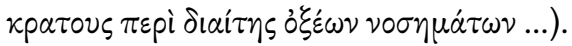




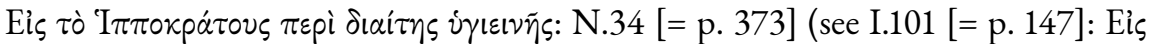

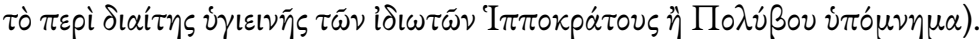

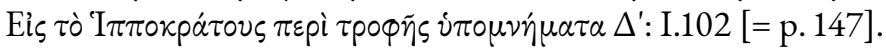

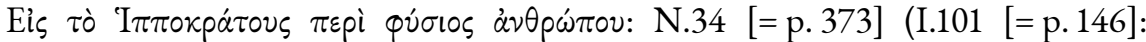

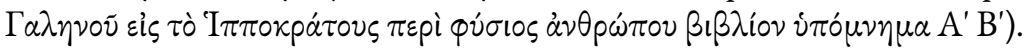

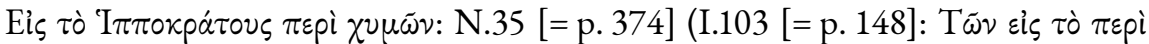

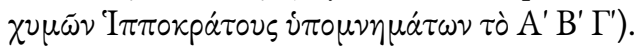

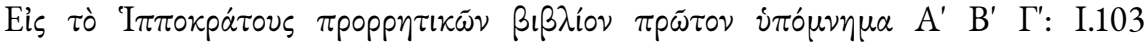
[= p. 149].

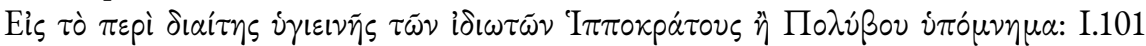

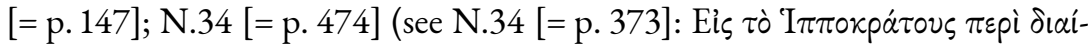
$\tau \eta \varsigma$ ن่ $\gamma(\varepsilon เ \nu \tilde{\eta} \varsigma)$.

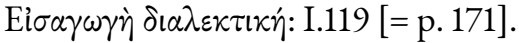

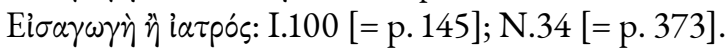

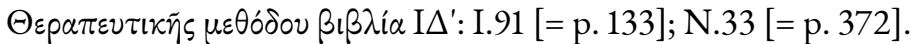

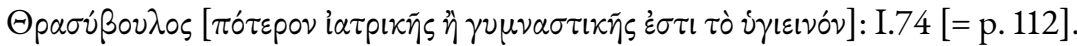

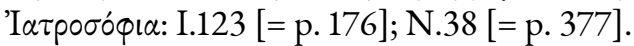

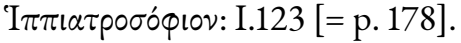

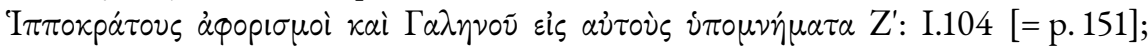
N.35 [= p. 374].

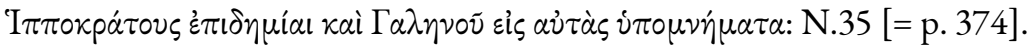

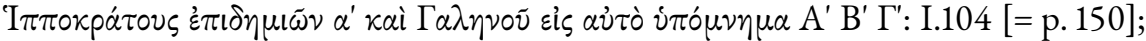

N.35 [= p. 374].

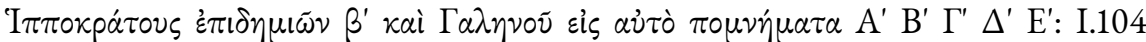
$[=$ p. 150]; N.35 [= p. 374].

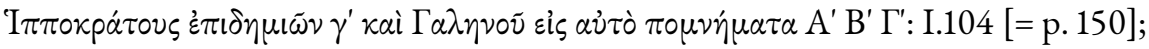
N.35 [= p. 374].

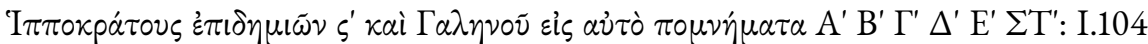
[= p. 150]; N.35 [= p. 374].

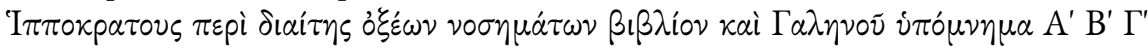

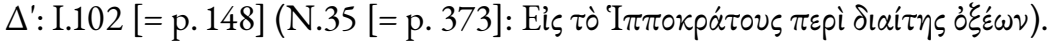

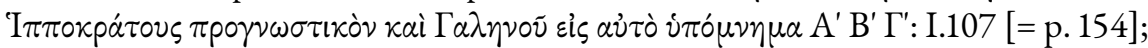
N.35 [= p. 374].

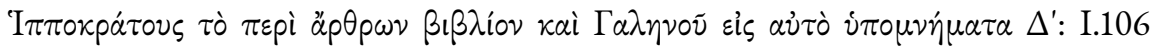
$[=$ p. 153].

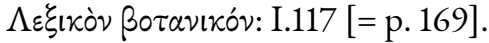

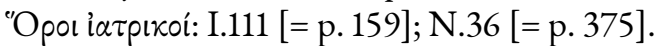

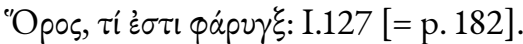

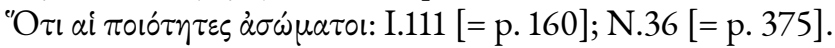

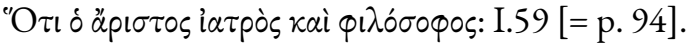




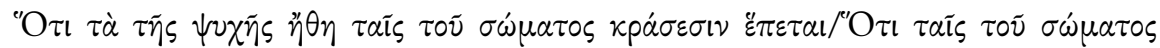

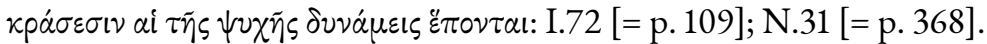

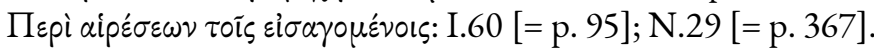

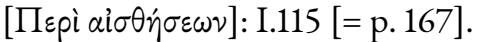

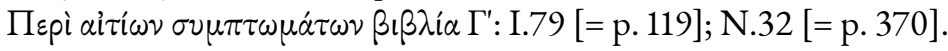

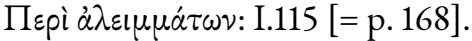

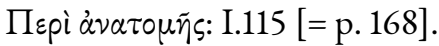

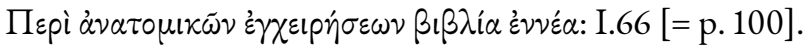

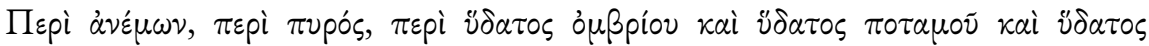

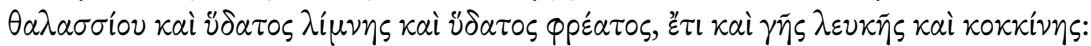
$\mathrm{I} .116[=\mathrm{p} .168]$.

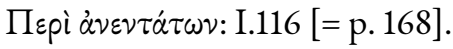

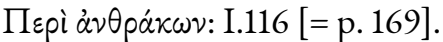

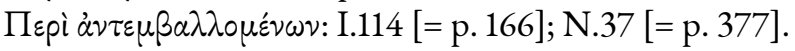

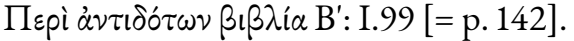

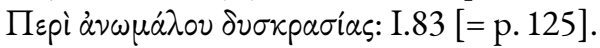

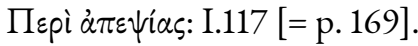

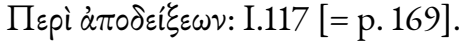

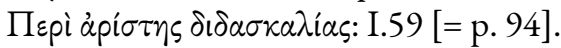

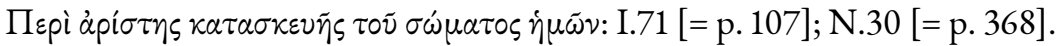

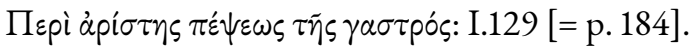

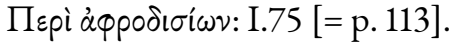

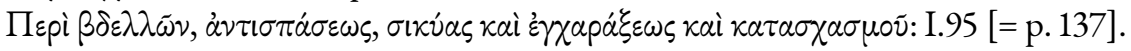

Пврі $\gamma \alpha \dot{\lambda} \alpha \kappa \tau o s: ~ I .117$ [= p. 170].

Пврі $\gamma \varepsilon \nu \varepsilon ́ \sigma \varepsilon \omega \varsigma: ~ I .117$ [= p. 170].

Пвpi yovoppoías: $\mathrm{I} .117$ [= p. 170].

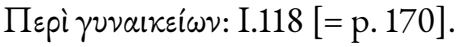

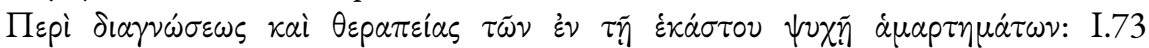
[= p. 110].

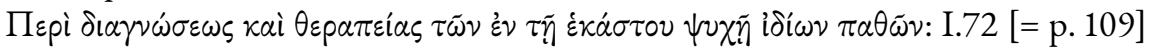

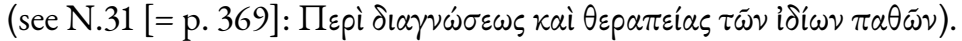

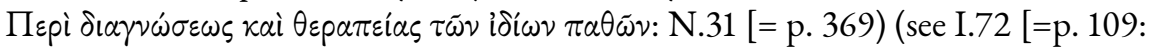

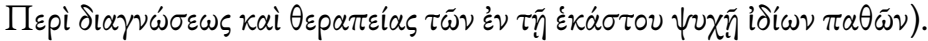

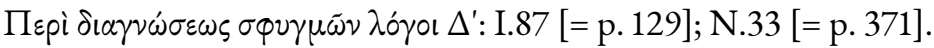

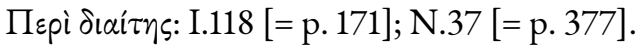

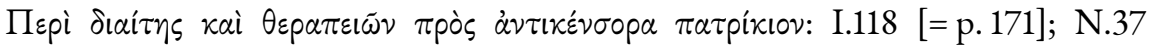
[= p. 377].

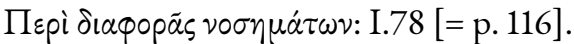

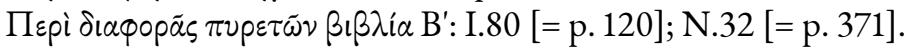

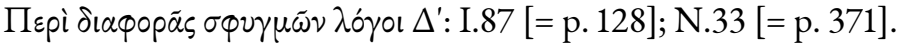

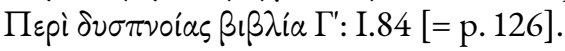




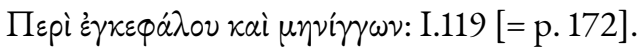

Пврі हे $\theta \tilde{\omega} v:$ I.119 [= p. 172].

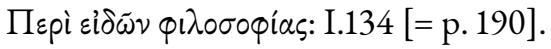

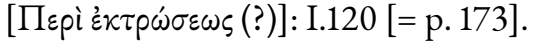

Пврі $\dot{\varepsilon} \lambda \kappa \tilde{\omega} v:$ I.120 [= p. 173].

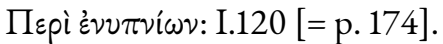

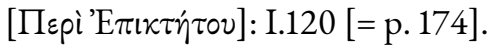

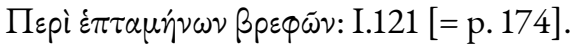

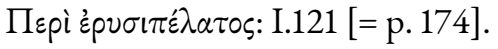

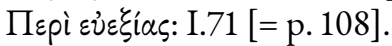

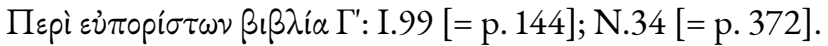

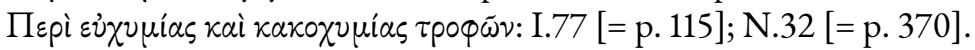

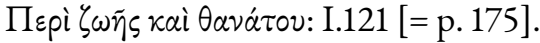

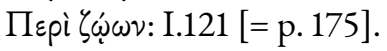

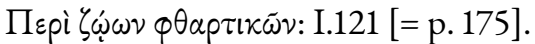

Пврі йं $\alpha \tau \circ$ : I.122 [= p. 175].

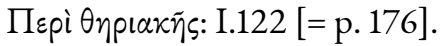

Пєрі Ө

Пврі $\theta \lambda \dot{\alpha} \sigma \mu \alpha \tau o s:$ I.122 [= p. 176].

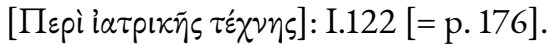

Пврі i $\alpha \tau \rho \tilde{\omega} \nu:$ I.123 [= p. 177].

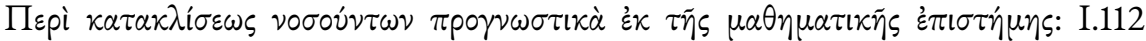
[= p. 162]; N.36 [= p. 376].

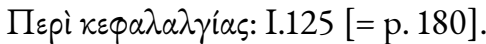

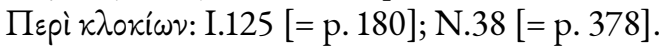

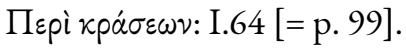

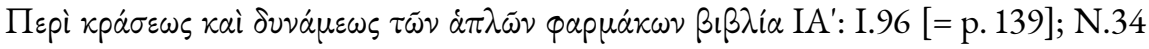
[= p. 372].

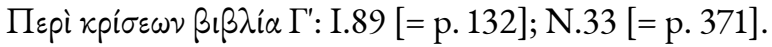

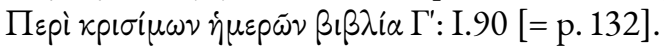

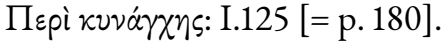

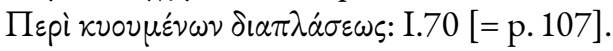

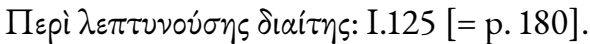

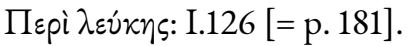

Пврі $\lambda \hat{i} \theta \omega \nu:$ I.126 [= p. 181]; N.38 [= p. 378].

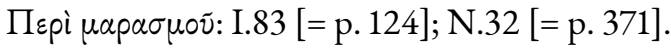

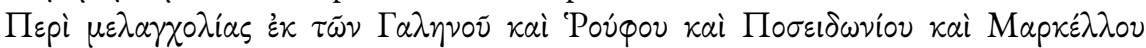

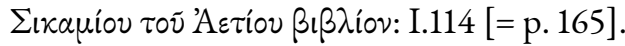

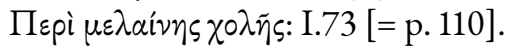

Пвpi $\mu \varepsilon \tau \dot{\alpha} \lambda \lambda \omega \nu$ : I.126 [= p. 181].

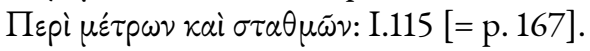




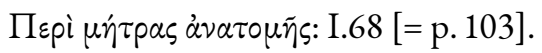

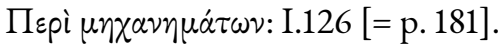

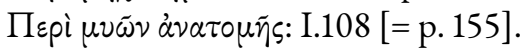

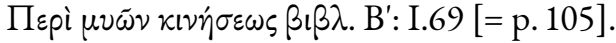

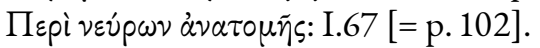

Пвpi vó $\omega \omega v:$ I.126 [= p. 181]; N.38 [= p. 378].

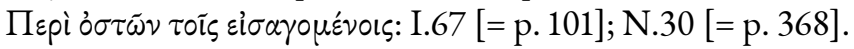

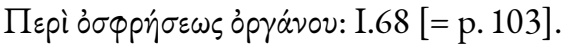

Пврі oü $\omega \nu:$ I.127 [= p. 182]; N.39 [= p. 379].

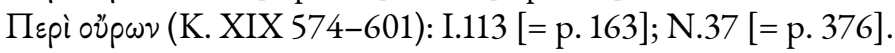

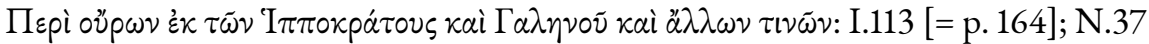
$[=$ p. 376].

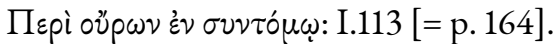

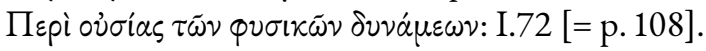

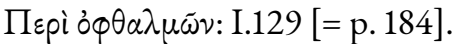

Пврі $\pi \lambda \hat{\eta} \theta$ ovs: I.82 [= p. 123].

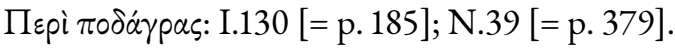

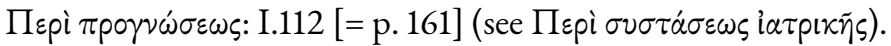

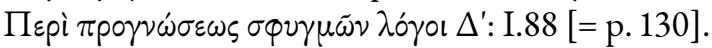

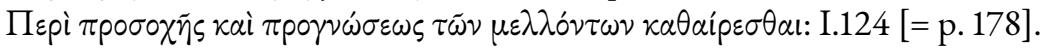

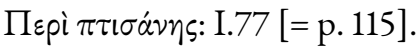

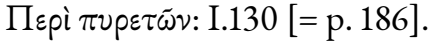

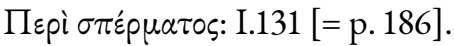

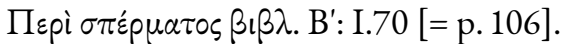

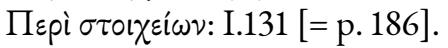

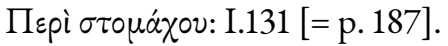

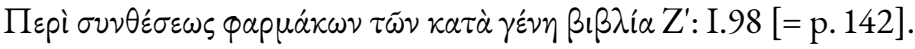

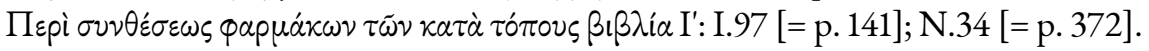

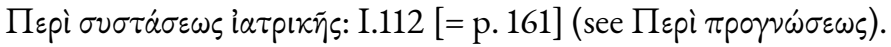

Пврі $\sigma \varphi v \gamma \mu \tilde{\omega} \nu:$ I.131 [= p. 187].

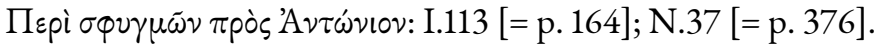

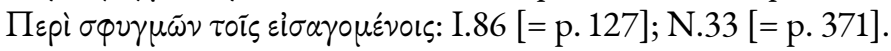

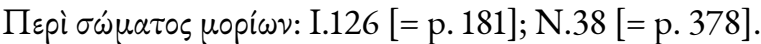

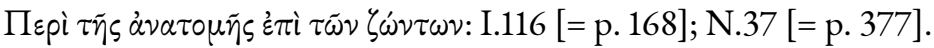

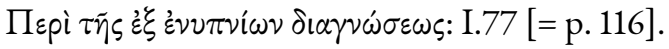

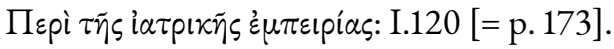

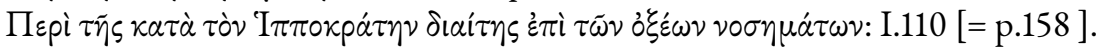

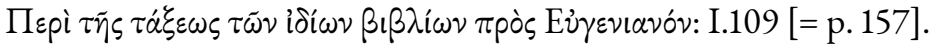

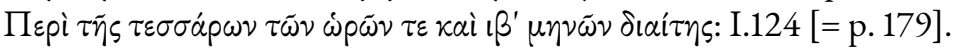

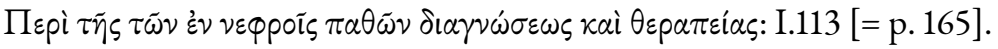

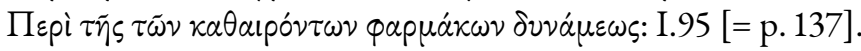




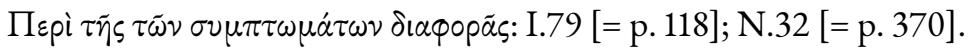

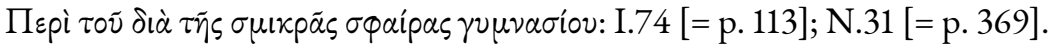

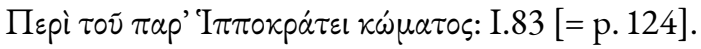

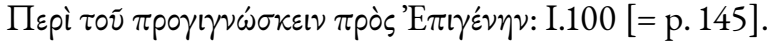

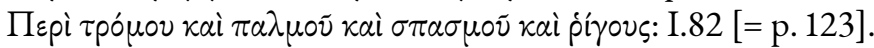

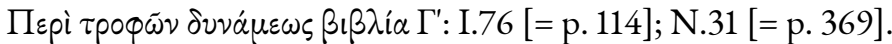

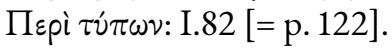

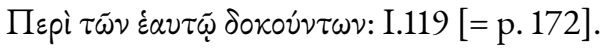

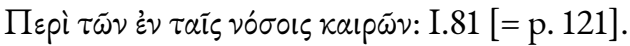

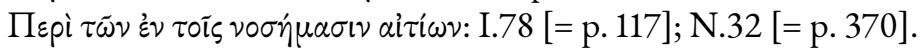

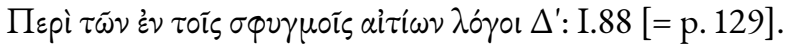

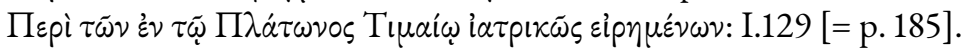

$\Pi \varepsilon p i \tau i \tilde{\omega} \nu \dot{\varepsilon} \pi \mathrm{i} \delta \dot{\varepsilon} \sigma \mu \omega \nu: \mathrm{I} .107$ [= p. 153].

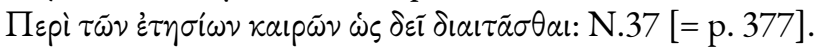

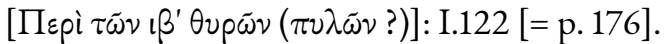

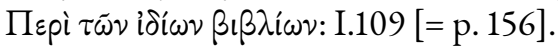

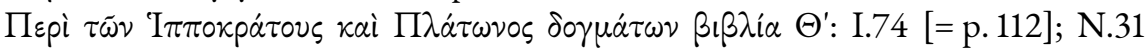

$[=$ p. 369].

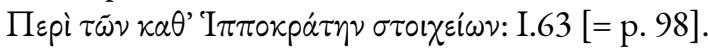

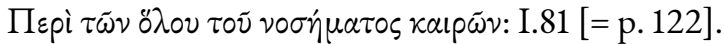

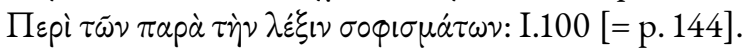

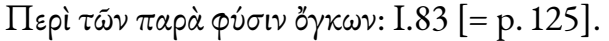

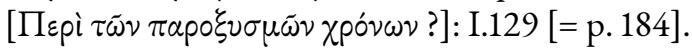

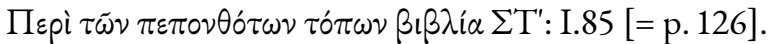

$\Pi \varepsilon p i \tau \tilde{\omega} \nu \tau \tilde{\eta} s \dot{\alpha} \nu \alpha \pi \nu \circ \tilde{\eta} s \alpha i \tau i \omega \nu:$ I.70 [= p. 105].

Пвpi va $\lambda i \omega v$ : I.132 [= p. 187].

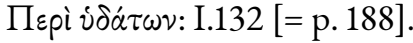

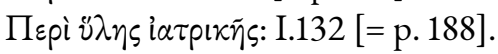

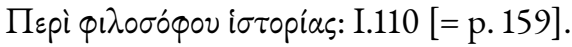

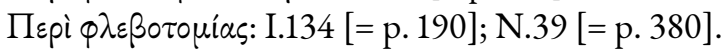

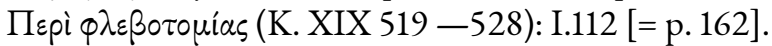

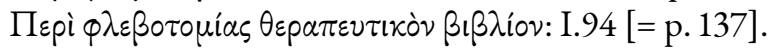

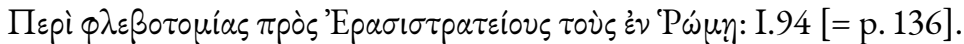

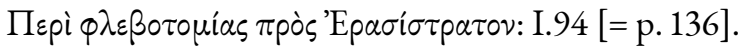

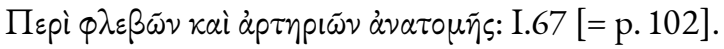

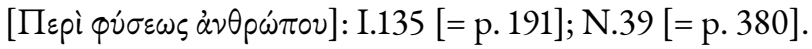

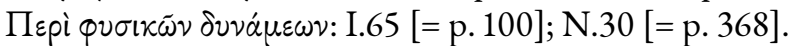

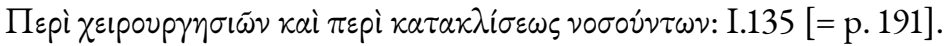

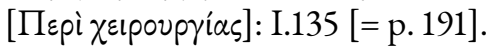

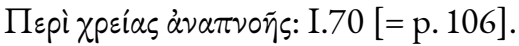

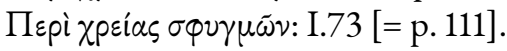




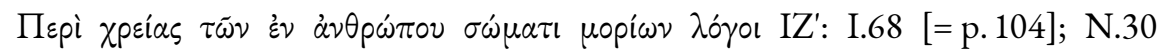
[= p. 368].

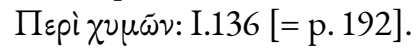

Пврі $\chi v \mu \tilde{\omega} \nu$ (K. XIX 485-496): I.111 [= p. 161].

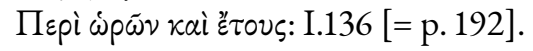

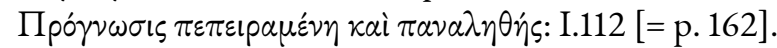

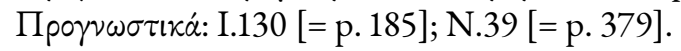

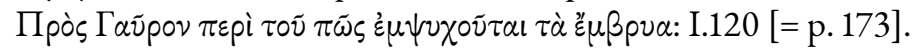

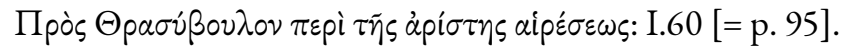

Прòs $\Lambda$ úxov: I.106 [= p. 152].

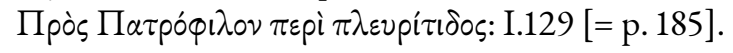

Прòs Патрó

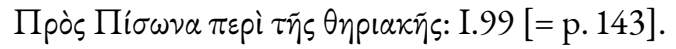

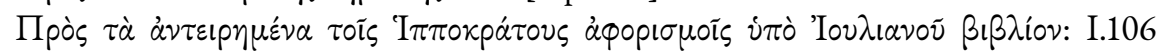
[= p. 153].

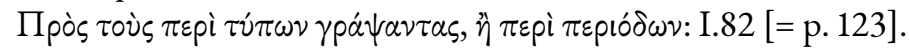

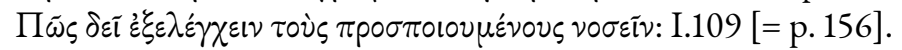

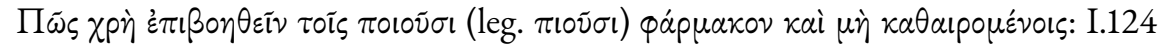
[= p. 179].

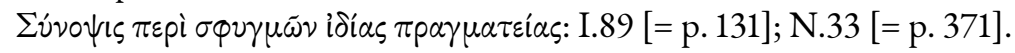

T'́x $\nu \eta$ i $\alpha \tau$ plkń: I.61 [= p. 96]; N.30 [= p. 367].

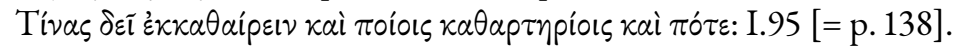

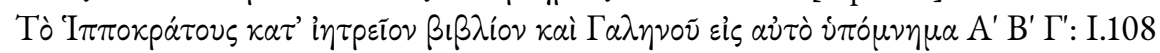
$[=$ p. 155].

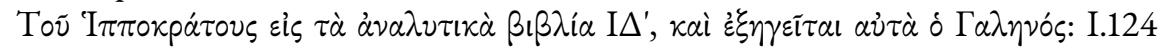
[= p. 178]; N.38 [= p. 378].

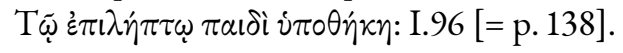

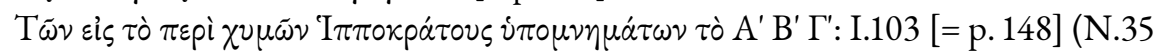

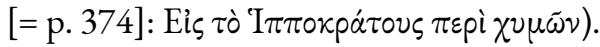

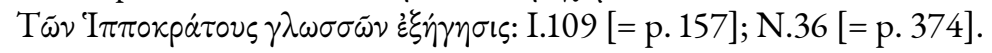

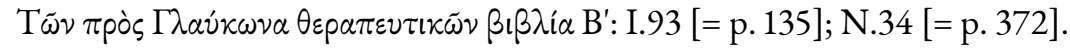

'Ү

Ф́́puaka: I.132 [= p. 188]; N.39 [= p. 379].

\section{Gregorius Nyssenus}

Excerpta varia: II.40 [= p. 254].

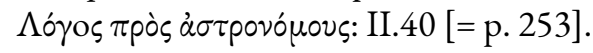

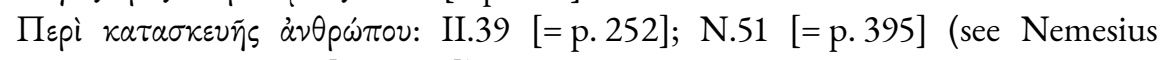
Emesenus $=$ II.66 [ $=$ p. 283]).

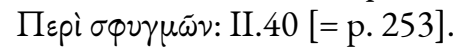




\section{Gregorius Theologus}

'A $\lambda \alpha ́ \tau ı$ : II.41 [= p. 254]; N.51 [= p. 395] (see Lucas = II.58 [= p. 272] and N.56 $[=$ p. 402]).

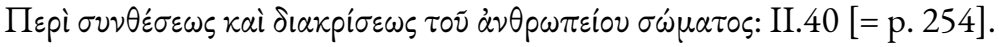

\section{Harpocration}

Excerpta varia: N.51 [= p. 396].

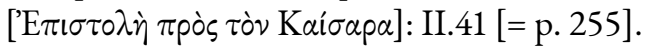

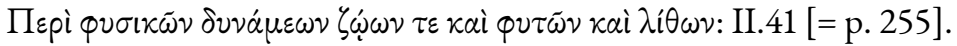

\section{Heliodorus}

Excerpta varia: II.42 [= p. 256].

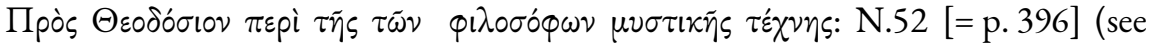

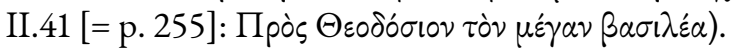

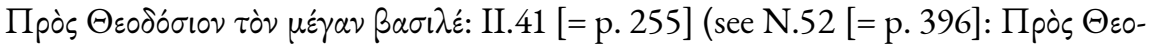

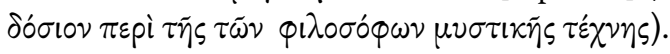

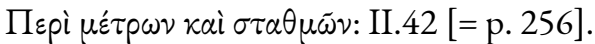

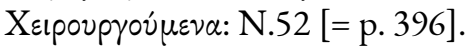

\section{Heraclius}

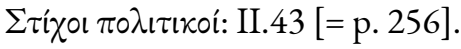

\section{Hermes Trismegistus}

Excerpta varia: II.47 [= p. 261]; N.54 [= p. 399].

Opera varia: II.43 [= p. 257]; N.52 [= p. 397].

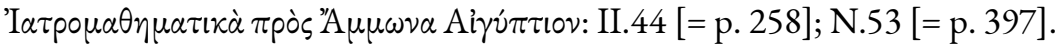

Kavóviov: II.43 [= p. 257].

Kvpavíøs: II.43 [= p. 257]; N.52 [= p. 397].

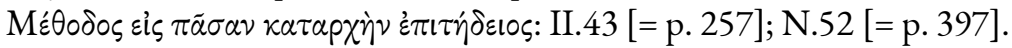

"Opyavov: II.43 [= p. 257].

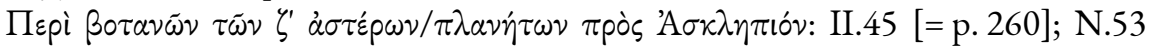
[= p. 399].

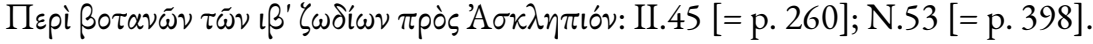

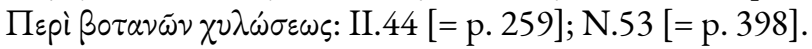

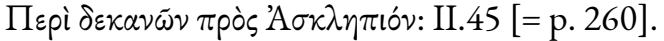

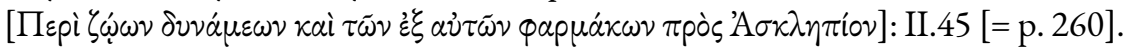

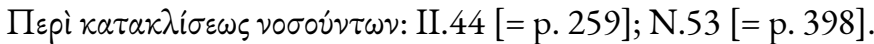

Пврі $\sigma \tau \alpha \theta \mu \tilde{\omega} \nu:$ II.45 [= p. 261].

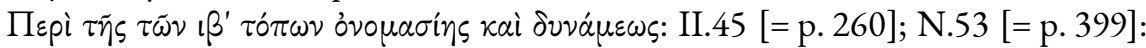

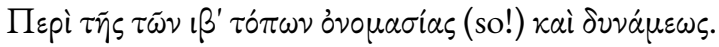

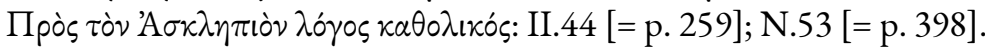

\section{Herodotus}

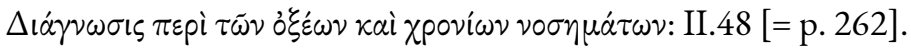

\section{Herophilus}

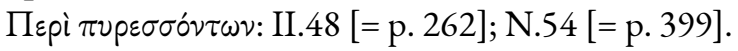


$\Sigma ф v \gamma \mu \omega \tilde{\nu}$ öpol: II.49 [= p. 262].

\section{Hierophilus Sophista}

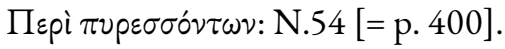

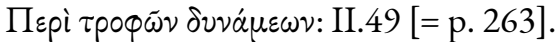

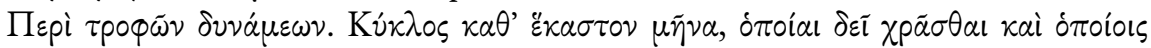
$\dot{\alpha} \pi \dot{\varepsilon} \chi \varepsilon \sigma \theta \alpha 1:$ II.49 [= p. 263].

\section{Hippokrates}

Excerpta varia: I.56 [= p. 89]; N.29 [= p. 363].

Indices et Lexica: I.57 [= p. 90].

Laterculi antiqui: I.57 [= p. 90].

Opera varia: I.3 [= p. 7]; N.25 [= p. 359].

'Афорıбноí: I.12 [= p. 19]; N.25 [= p. 359].

Гvvalksíwv A' B': I.29 [= p. 53].

$\Delta \iota \alpha \theta \dot{\eta} \kappa \eta:$ I.39 [= p. 74].

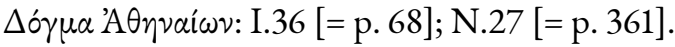

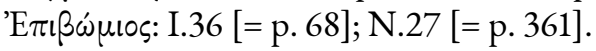

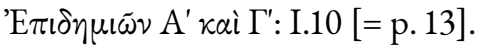

'E $\pi i \delta \eta \mu i \omega \nu \nu$ B'. $\Delta^{\prime}-Z$ ': I.19 [= p. 28].

'E $\pi\llcorner\sigma \tau 0 \lambda \alpha i:$ I.36 [= p. 68]; N.27 [= p. 361].

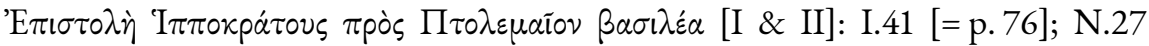
[= p. 362].

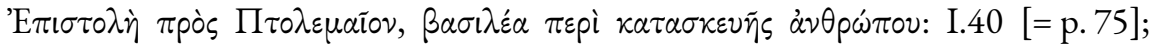

N.27 [= p. 361].

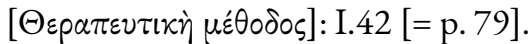

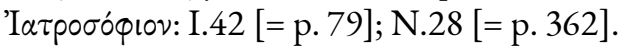

'I $\pi \pi \iota \alpha$ pıxá: I.43 [= p. 80].

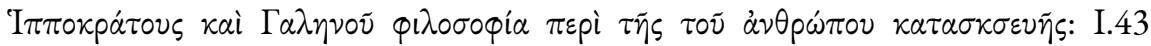
[= p. 80].

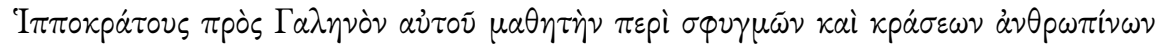
$\sigma \omega \mu \dot{\alpha} \tau \omega v:$ I. 47 [= p. 85].

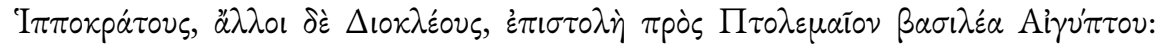
I.40 [= p. 74].

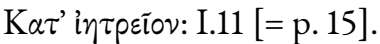

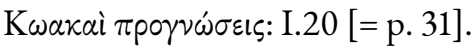

$\Lambda \varepsilon \xi$ ६xòv $\tau \tilde{\omega} \nu \varepsilon i \delta \tilde{\omega} v:$ I.40 [= p. 74].

$\Lambda o ́ \gamma o s \tau \tilde{\omega} \nu \mu \eta \nu \tilde{\omega} v:$ I.43 [= p. 81].

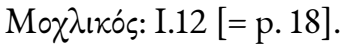

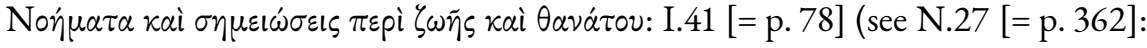

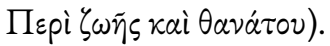

Nópos: I.18 [= p. 26].

"Opros: I.17 [= p. 25]. 


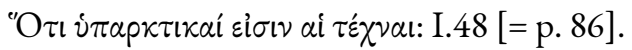

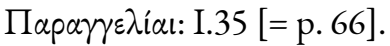

Пвpi $\alpha \gamma \mu \tilde{\omega} \nu:$ I.11 [= p. 16].

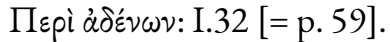

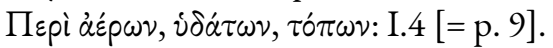

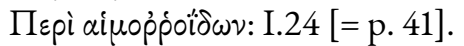

Пвpi ai $\theta \dot{\eta} \sigma \varepsilon \omega \nu:$ I.39 [= p. 73]; N.27 [= p. 361].

Пврі $\dot{\alpha} \lambda \varepsilon \varphi \mu \mu \alpha \dot{\tau} \tau \omega v:$ I.39 [= p. 73].

Пвpi à $\lambda$ óns: I.39 [= p. 73].

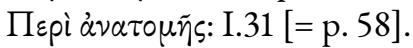

Пврі $\alpha \nu \alpha \tau o \mu \tilde{\eta} \varsigma \tau \tilde{\omega} \nu \zeta \omega \dot{\omega} \nu \tau \omega \nu:$ N.27 [= p. 361] (with a reference [Zu Kat. I S. 39] $[=$ p. 73] that does not correspond).

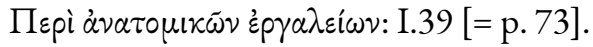

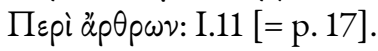

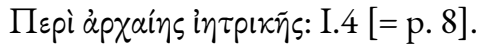

Пвpi à óp $\omega \nu$ : I.30 [= p. 54].

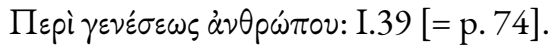

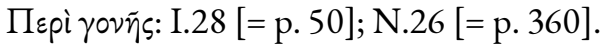

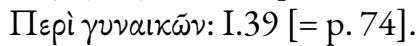

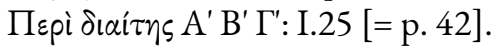

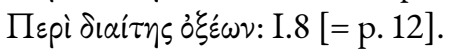

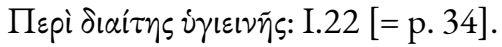

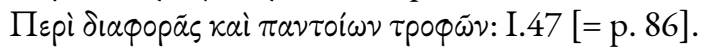

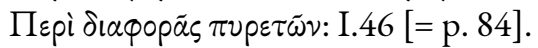

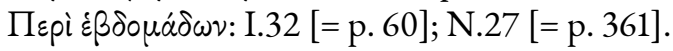

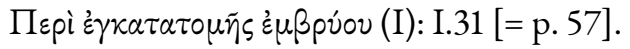

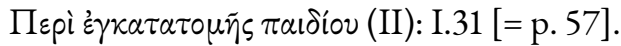

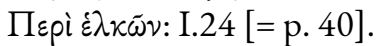

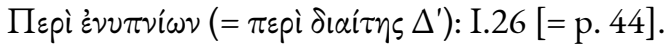

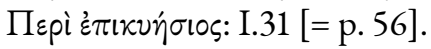

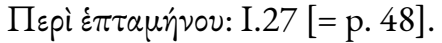

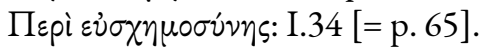

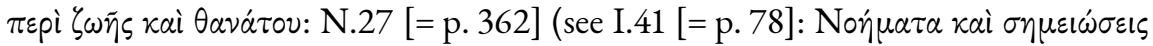

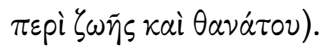

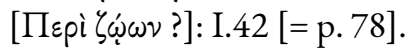

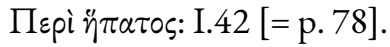

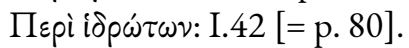

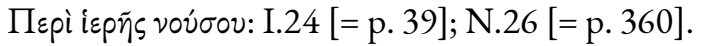

Пвpi in $\tau$ pov: I.34 [= p. 65].

Пврі кардіns: I.33 [= p. 62].

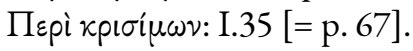




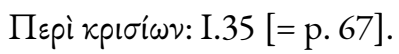

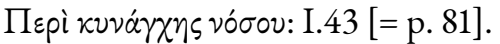

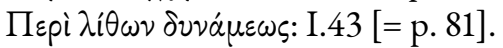

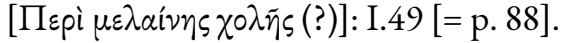

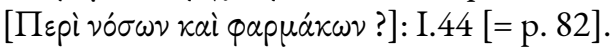

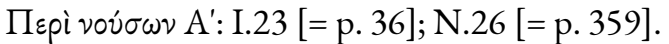

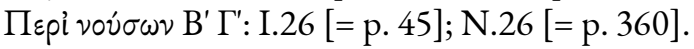

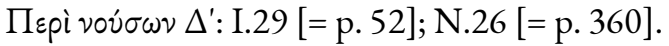

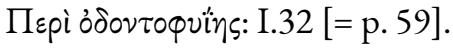

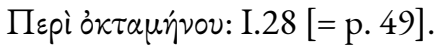

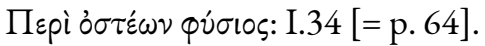

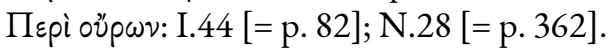

Пврі őభıs: I.34 [= p. 63].

Пвpi $\pi \alpha \theta \tilde{\omega} v$ : N.28 [= p. 363] (with a reference [Zu Kat. I S. 44] [= pp. 82-83] that does not correspond).

Пврі $\pi \alpha \theta \tilde{\omega} \nu$ (L. VI 208-270): I.23 [= p. 37]; N.26 [= p. 360].

Пврі $\pi \alpha \rho \theta \varepsilon v i \omega v: I .30$ [= p. 55]; N.26 [= p. 361].

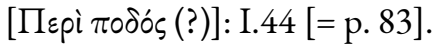

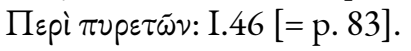

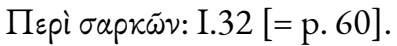

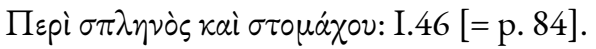

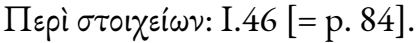

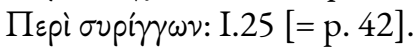

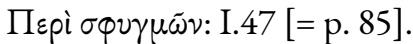

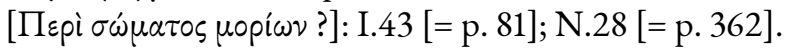

Пврі $\tau \dot{\chi} \chi \nu \eta s:$ I.20 [= p. 32].

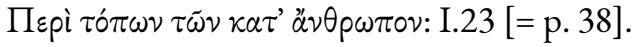

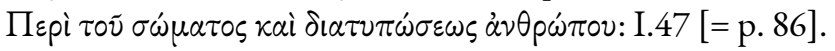

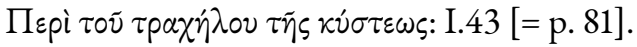

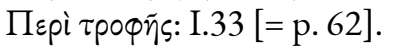

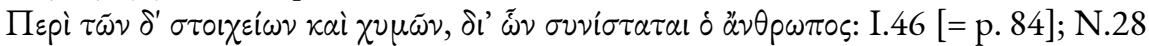
$[=$ p. 363].

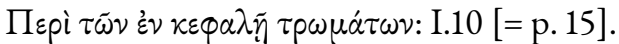

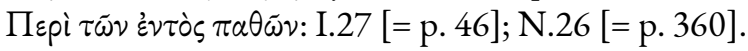

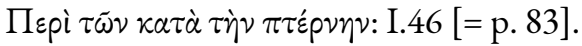

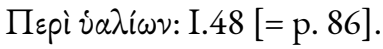

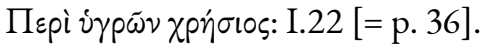

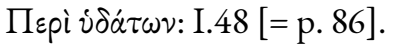

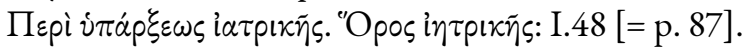

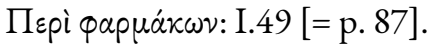

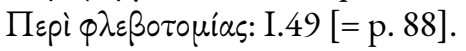




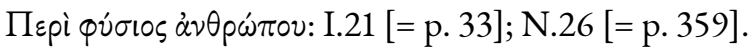

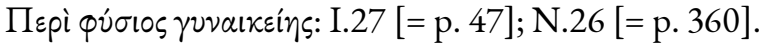

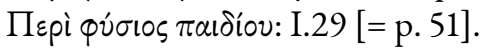

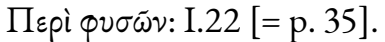

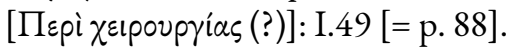

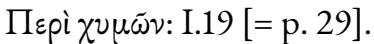

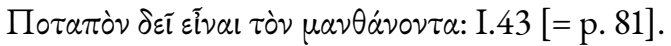

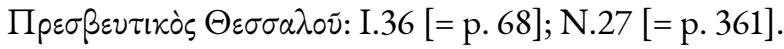

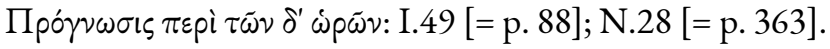

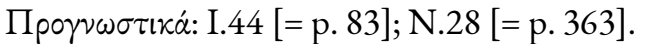

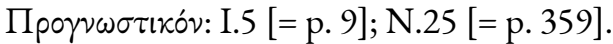

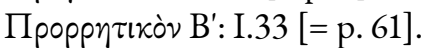

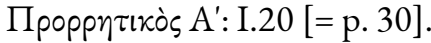

Фápuaka: I.48 [= p. 87]; N.28 [= p. 363].

\section{Hypatus}

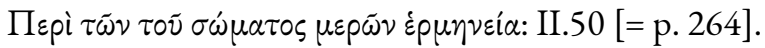

\section{Iacobus Psychrestus}

Excerpta: II.50 [= p. 264].

\section{Ioannes Alexandrinus (nicht Philoponus)}

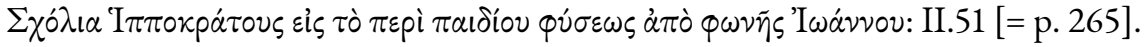

\section{Ioannes Antiochenus Archiatrus}

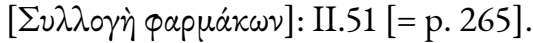

\section{Ioannes Archiatrus}

П $\alpha \tau \tau o i ́ \omega \nu \pi \alpha \theta \tilde{\omega} \nu \theta \varepsilon p \alpha \pi \varepsilon i \alpha:$ II.52 [= p. 266]; N.54 [= p. 400].

Ioannes Chrysostomus (N.54 [= p. 400]: $V g l$. Nachtrag über Chrysostomus = N.46 $[=$ p. 389]).

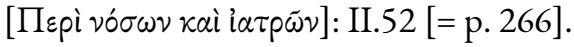

\section{Ioannes Chumnos}

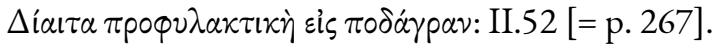

\section{Ioannes Damascenus}

Excerpta: II.53 [= p. 267].

Ioannes Dioecetas Constantinopolitanus (N.54 [= p. 400]: »Doch wohl $\Delta$ เol$\kappa \eta \tau \dot{n} \varsigma \ll$ Treu)

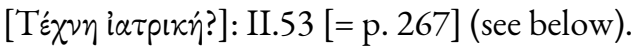

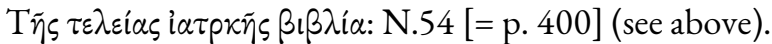

\section{Ioannes Episcopus Prisdrianensis}

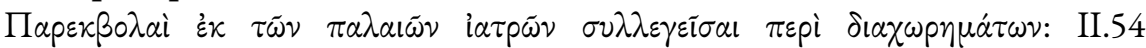
$[=$ p. 268]; N.55 [= p. 400].

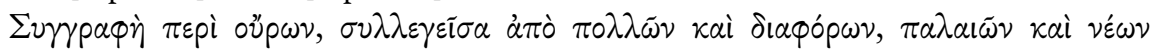

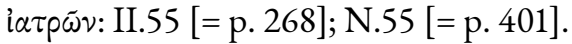




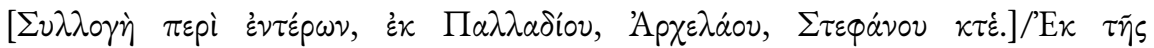

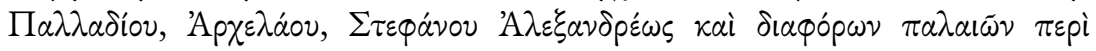
$\dot{\varepsilon} \nu \tau \dot{\varepsilon} \rho \omega \nu:$ II. 55 [= p. 269]; N.55 [= p. 401].

\section{Ioannes Esdra}

Пвpi oṽ $\rho \omega v:$ II.53 [= p. 267].

\section{Ioannes Iatrosophista}

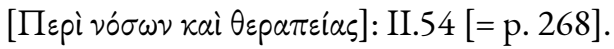

\section{Ioannes Philoponus Alexandrinus}

Пepi $\pi v p \varepsilon \tau \tilde{\omega} v:$ II.50 [= p. 265].

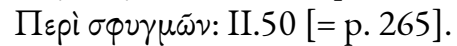

\section{Ioannes Pothus Pediasimus}

( $V g l$ Krumbacher, Sitz. Ber. d. Münch. Akad. d. Wiss. 1892 p. 350): II.54 [= p. 268].

\section{Ioannes Spensatus}

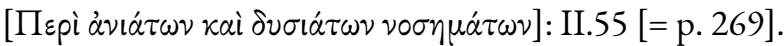

\section{Ioannes Staphidaces}

$\sum\langle\dot{v} \theta \varepsilon \sigma\llcorner\zeta \delta 1 \alpha \varphi o ́ \rho \omega \nu \pi \rho \dot{\xi} \xi \varepsilon \omega \nu$ : II.55 [= p. 269]; N.55 [= p. 401].

\section{Ioannes Zacharias Actuarius}

(S. unter Zacharias)

\section{Iustus}

Excerpta: II.56 [= p. 270]; N.56 [= p. 401].

\section{Karastonus}

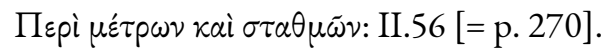

Kyranus ( $V g l$. Harpocration und Hermes Trismegistus)

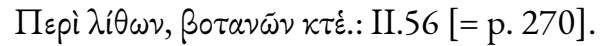

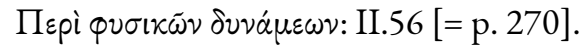

\section{Lampudes}

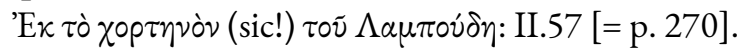

Leo

Excerpta: II. 57 [= p. 271].

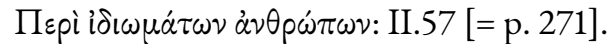

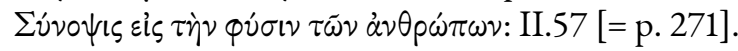

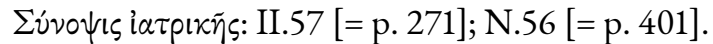

\section{Libanius}

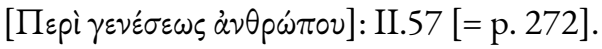

\section{Logadius}

Excerpta: II.58 [= p. 272]; N.56 [= p. 402].

Lucas $(\mathrm{Vgl}$. Gregorius Theol[ogus] = II.41 [= p. 254])

Excerpta varia: II.58 [= p. 273]; N.56 [= p. 402].

$\sum \kappa \varepsilon v \alpha \sigma i \alpha a ̉ \lambda \alpha \tau i ́ o v:$ II.58 [= p. 272]; N.56 [= p. 402]. 


\section{Lycus}

Excerpta varia: II.59 [= p. 273].

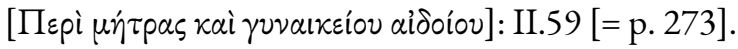

\section{Macarius Magnes}

Пвpi oủpwv: II.59 [= p. 273].

Macarius Maximus (N.56 [= p. 403]: d. i. uaxápıos (beatus) Maximus, scil[icet] Confessor)

Пвpi $\psi v x \tilde{\eta} s:$ II.59 [= p. 274]; N.56 [= p. 403].

\section{Magnus Emesenus}

Excerpta: II.60 [= p. 275].

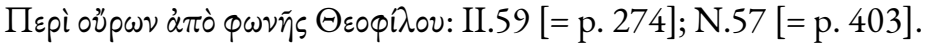

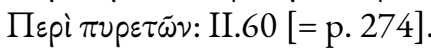

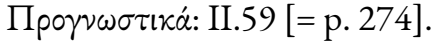

Mangens (?) (= Macarius Magnes oder Magnus Emesenus?)

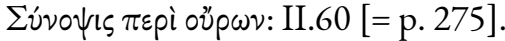

\section{Manuel Comnenus}

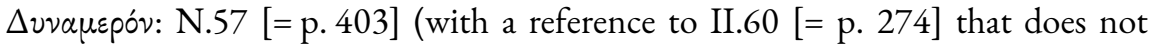
correspond).

\section{Marcellinus}

Пврі $\sigma \varphi v \gamma \mu \tilde{\omega} v:$ II.60 [= p. 275]; N.57 [= p. 403].

\section{Marcellus Sidetes}

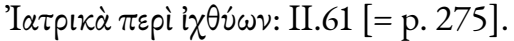

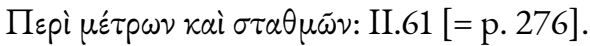

\section{Marcianus}

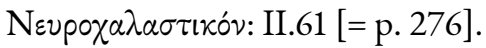

\section{Marianus}

Excerpta: II.62 [= p. 276]; N.57 [= p. 404].

Maximus (Planudes?) (N.57 [= p. 404]: »Schwerlich von Planudes« Treu. Vgl. Nachtrag zu Meletius Excerpta varia = N. 59 [= p. 406])

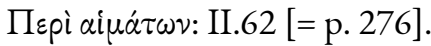

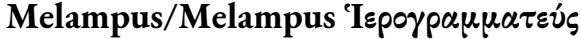

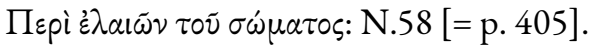

[Пврі $\pi \alpha \lambda \mu \tilde{\omega} \nu]:$ II.62 [= p. 277]. See below.

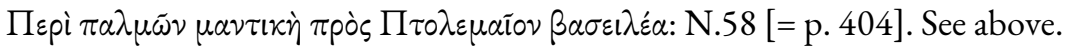

\section{Meletius Monachus}

Excerpta varia: II.64 [= p. 279]; N.59 [= p. 406].

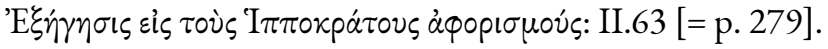

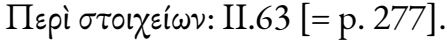

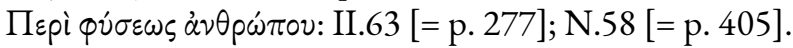

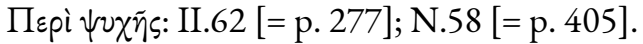




\section{Menander Iatrosophista}

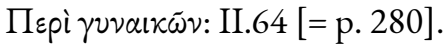

\section{Mercurius Monachus}

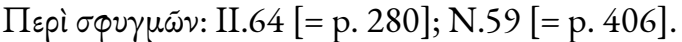

\section{Methodius Causocalybitus}

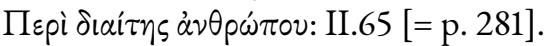

Methodius Patriarchus (N.59 [= p. 406]: d. i. »Patriarch«, nämlich von Konstantinopel, »also zu schreiben Patriarcha« Treu)

Excerpta varia: II.65 [= p. 281].

\section{Metrodora}

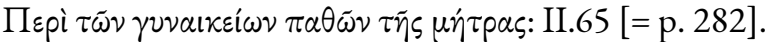

\section{Mnesitheus Cyzicenus}

Excerpta varia: II.65 [= p. 282].

\section{Moschion}

\section{Muscio}

$\Upsilon \pi 0 \theta \tilde{\eta} \kappa \alpha l:$ II.66 [= p. 282].

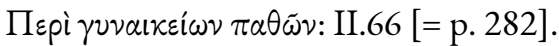

Nemesius Emesenus ( $\mathrm{Vgl}$. Gregorius Nyssenus = II.39 [= p. 252])

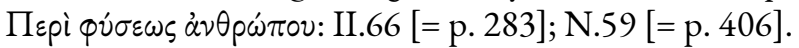

\section{Neophytus Prodromenus}

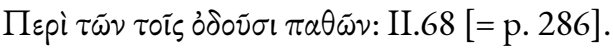

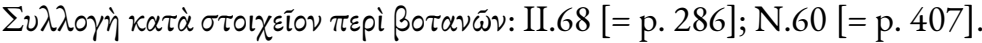

\section{Nepualius}

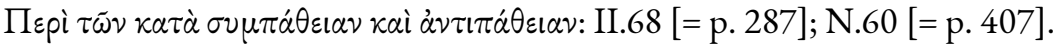

\section{Nicolaus Myrepsus}

Excerpta: II.69 [= p. 288].

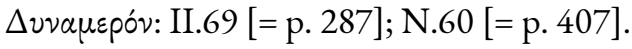

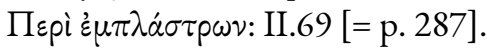

\section{Nicomedes}

Eis I $\pi \pi \circ$ кра́ $\tau \eta v:$ II.69 [= p. 288].

\section{Nicomedes Iatrosophista}

Lexicon medicum: II.69 [= p. 288].

\section{Ocianus}

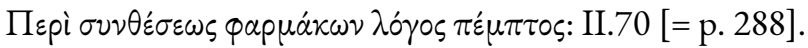

\section{Olymnius Alexandrinus}

Excerpta: II.70 [= p. 289].

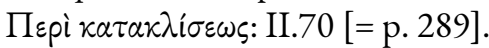

\section{Oribasius}

Excerpta varia: II.73 [= p. 293]; N.61 [= p. 408].

Opera varia: II.70 [= p. 289]; N. 60 [= p. 408].

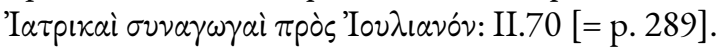




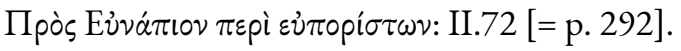

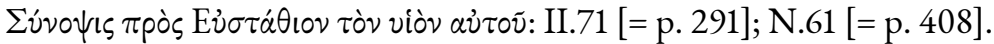

\section{Orpheus}

Пврі $\lambda i ̂ \omega \nu$ : II.75 [= p. 294].

\section{Palladius}

Excerpta varia: II.76 [= p. 297].

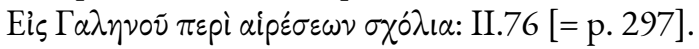

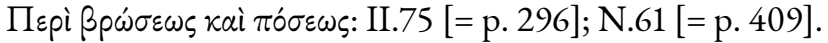

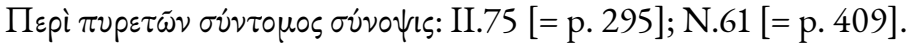

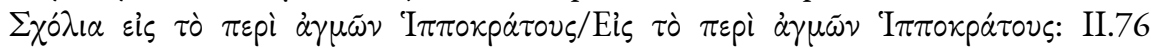
[= p. 296]; N.61 [= p. 409].

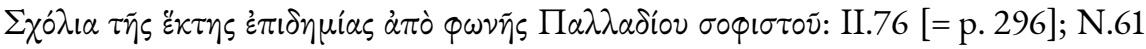
[= p. 409].

\section{Pamphilus}

Excerpta: II.76 [= p. 297] (N.62 [= p. 409]: »Nur in Receptensammlungen« Treu).

\section{Pancharius}

Excerpta: II.77 [= p. 297].

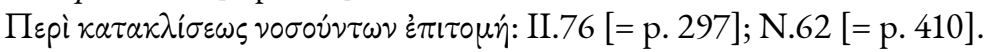

\section{Paulus Aegineta}

De venenis: II.78 [= p. 300].

Excerpta varia: II.79 [= p. 303]; N.62 [= p. 411].

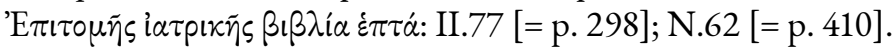

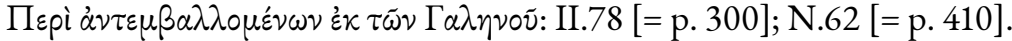

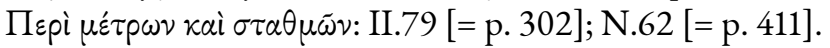

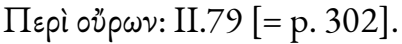

\section{Paulus Italus}

Excerpta: II.81 [= p. 305].

\section{Paulus Nicaeensis}

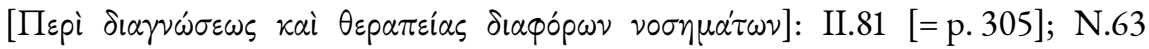
$[=$ p. 411].

\section{Perzoe}

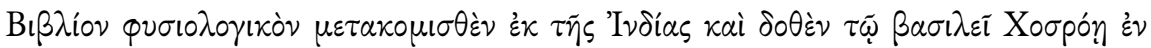

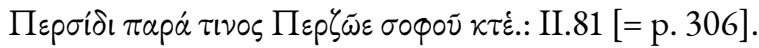

\section{Petosiris}

Excerpta: II.82 [= p. 307].

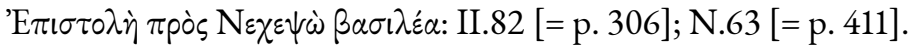

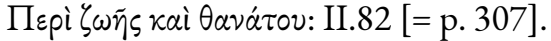

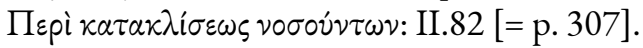

\section{Philagrius}

Excerpta varia: II.83 [= p. 308]. 


\section{Philaretus}

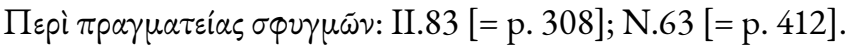

\section{Philippus Xerus}

$\sum \nu \nu \theta \varepsilon ́ \sigma \varepsilon ı \varsigma:$ N.63 [= p. 412].

\section{Philo}

Excerpta varia: II.85 [= p. 308]; N.63 [= p. 412].

\section{Philo, Herennius}

$\Gamma \nu \tilde{\omega} \sigma \iota \varsigma \tau \tilde{\omega} \nu \dot{\eta} \lambda \iota \kappa \iota \tilde{\omega} \nu:$ II.85 [= p. 309].

\section{Philumenus}

Excerpta varia: II.85 [= p. 309].

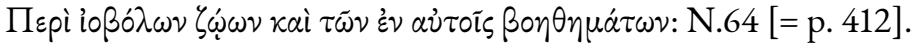

\section{Photius Monachus}

Carmina: II.85 [= p. 309].

Excerpta: II. 86 [= p. 309].

\section{Porphyrius}

Excerpta varia: II.86 [= p. 310].

\section{Posidonius}

\section{Proclus}

Excerpta varia: II.86 [= p. 310].

Excerpta: II.87 [= p. 310].

\section{Protagoras}

Protagorae [Pythagorae] excerpta ex iatromathematica Mercurii et Petosiridis etc. astrologia (S. oben Petosiris Excerpta [II.82] [= p. 307]): II.87 [= p. 310].

\section{Ptolemaeus}

Excerpta varia: II.87 [= p. 311]; N.64 [= p. 413].

\section{Pythagoras}

Protagorae [Pythagorae] excerpta ex iatromathematica Mercurii et Petosiridis etc. astrologia (S. oben Petosiris Excerpta [II.82] [= p. 307]): II.88 [= p. 311].

$\Psi \tilde{\eta} \varphi \circ:$ II.87 [= p. 311]; N.64 [= p. 413].

\section{Romanus}

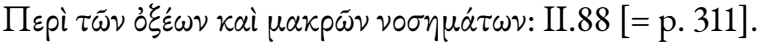

\section{Rufus}

Excerpta varia: II.91 [= p. 316]; N.65 [= p. 415].

Opera varia: N.64 [= p. 413].

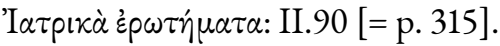

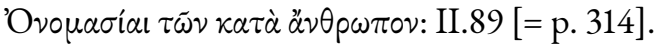

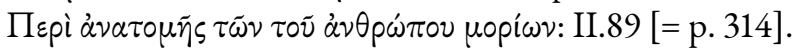

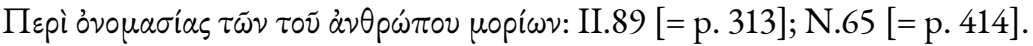

Пepi ò $\sigma \tau \tilde{\omega} v:$ II.90 [= p. 315].

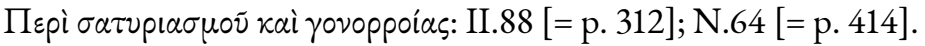

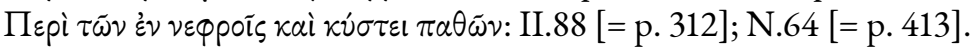




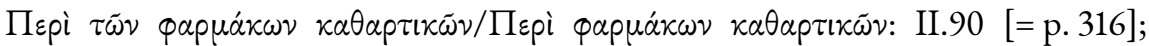
N.65 [= p. 414].

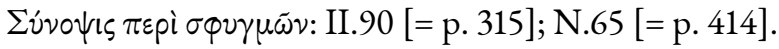

\section{Salomo}

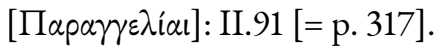

\section{Severianus}

Opıruoí: II.91 [= p. 317].

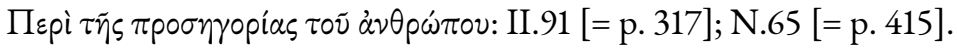

\section{Severus}

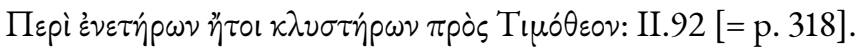

\section{Socrates}

Excerpta: II.92 [= p. 318].

\section{Soranus}

Excerpta varia: II.94 [= p. 321].

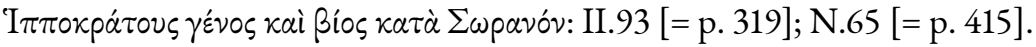

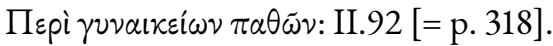

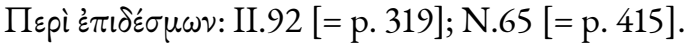

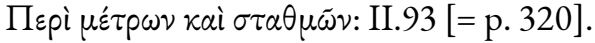

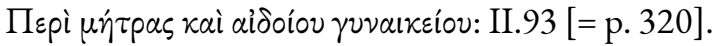

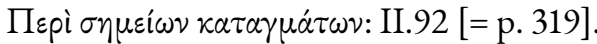

\section{Splenius Philosophus}

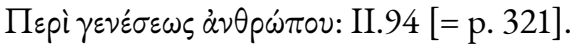

\section{Stephanus Alexandrinus}

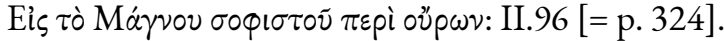

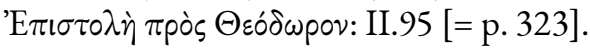

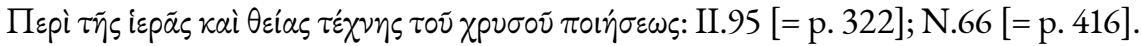

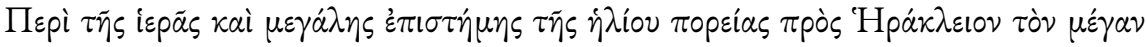

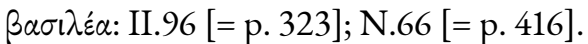

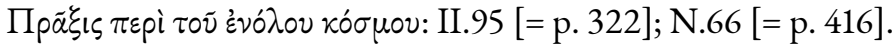

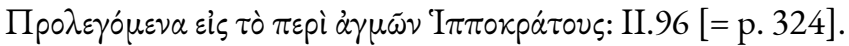

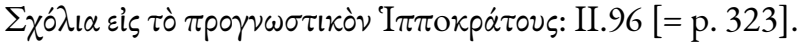

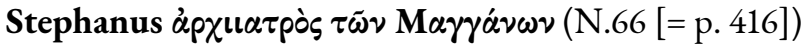

Excerpta varia: II.98 [= p. 327].

'E $\pi$ $\sigma \tau 0 \lambda \dot{\eta}:$ II.98 [= p. 327].

\section{Stephanus Atheniensis}

Excerpta varia: II.98 [= p. 327].

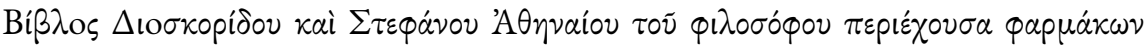

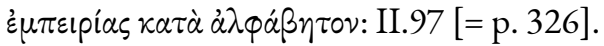

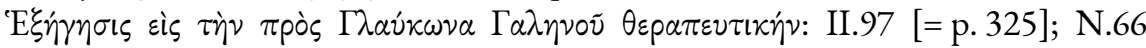
$[=\mathrm{p} .416]$.

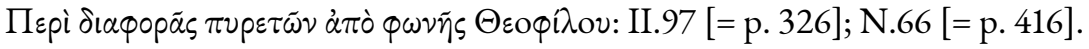




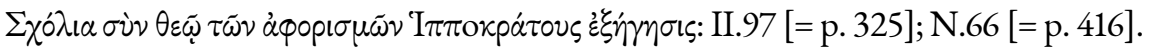

\section{Stephanus Magnes}

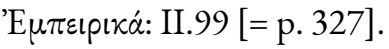

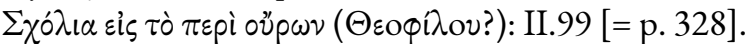

\section{Symeon S[anctus] Mamas}

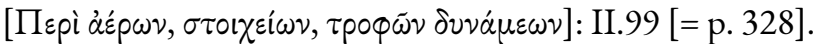

\section{Syncellus}

Excerpta: II.99 [= p. 328$] ;$ N.66 [= p. 417].

\section{Synesius}

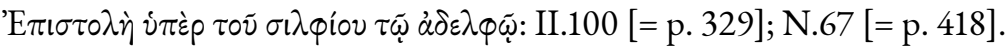

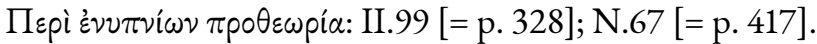

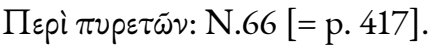

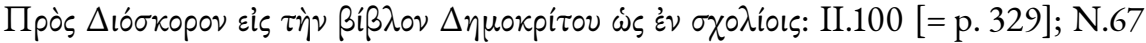
[= p. 418].

Taronitus (?)(see N.47 [= p. 389]: Constantinus Taronites)

Excerpta: II.100 [= p. 329]; N.67 [= p. 418].

Theodoretus (N.67 [= p. 418])

'A $\nu \tau i \delta \circ \tau \alpha:$ II.100 [= p. 330].

Theodorus (N.67 [= p. 418])

Excerpta: II.100 [= p. 330].

\section{Theophilus}

Excerpta varia: II.106 [= p. 336]; N.68 [= p. 419].

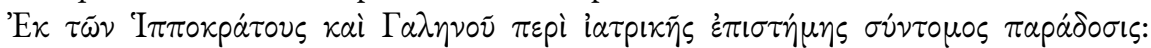
II.105 [= p. 335]; N.68 [= p. 419].

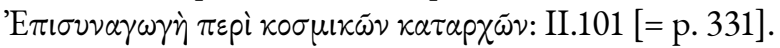

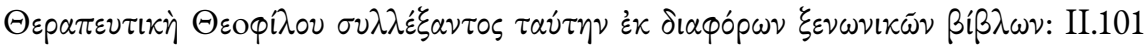
[= p. 331].

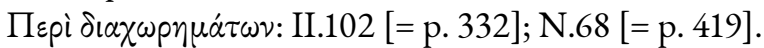

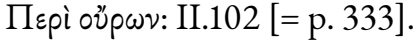

Пврі $\sigma \varphi v \gamma \mu \tilde{\omega} v:$ II.101 [= p. 331].

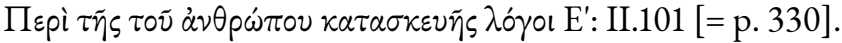

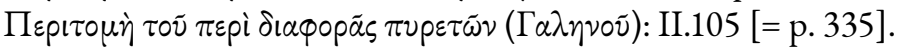

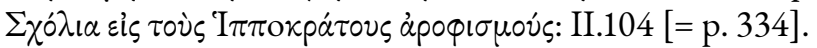

\section{Theophylactus Simocatta}

Versus in Galenum: II.106 [= p. 336].

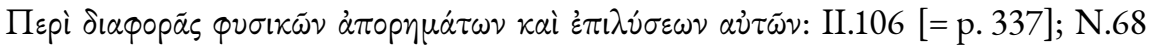
[= p. 419].

\section{Theopompus}

Excerpta: II.106 [= p. 337].

\section{Theosebius}

Excerpta: II.106 [= p. 337]. 


\section{Timocrates}

Excerpta: II.107 [= p. 337]

\section{Timotheus Gazaeus}

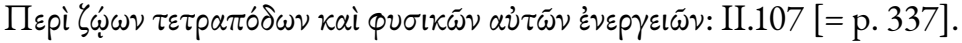

Vincentius Damodes (N.68 [= p. 419])

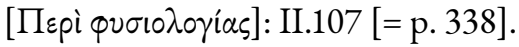

\section{Xenocrates}

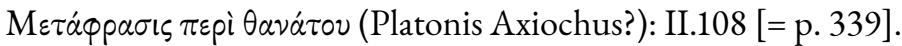

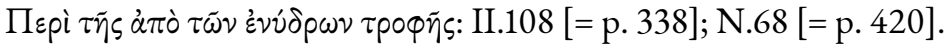

\section{Ioannes Zacharias Actuarius}

Excerpta varia: II.111 [= p. 344]; N.69 [= p. 420].

Opera varia: II.108 [= p. 339].

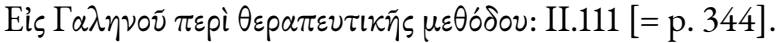

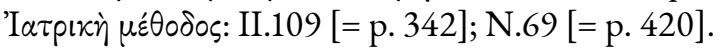

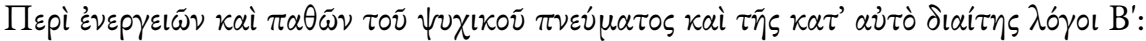

II.108 [= p. 339].

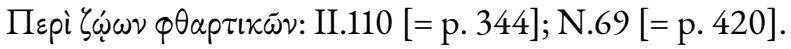

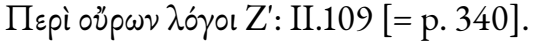

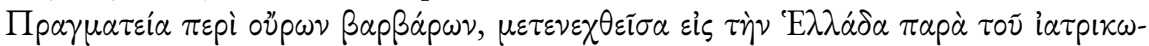

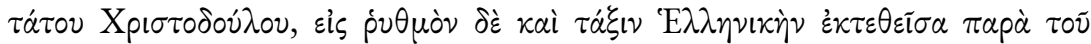

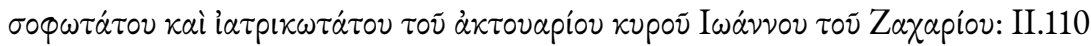
[=p. 344].

\section{Zenarius}

Excerpta: II.111 [= p. 345].

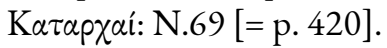

\section{Zosimus Panopolita}

Excerpta/Excerpta varia: II.112 [= p. 346]; N.69 [= p. 421].

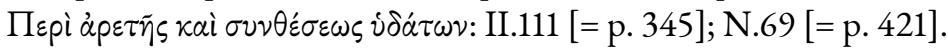

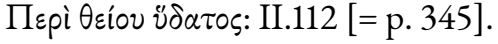

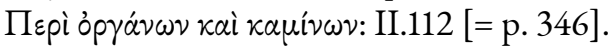

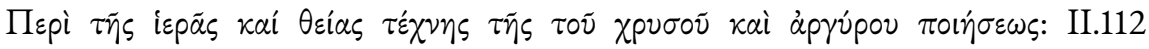
$[=$ p. 346]. 


\section{Latin titles}

\section{Abraham}

Excerpta varia: II.3 [= p. 201]; N.42 [= p. 383].

\section{Adamantius}

De palpitationibus: II.3 [= p. 201]; N.42 [= p. 383].

De ventis: II.3 [= p. 201].

Excerpta varia: II.3 [= p. 201].

\section{Aelianus}

Epistula anepigrapha: II.4 [= p. 202].

\section{Aelius Promotus}

De animalibus virus eiaculantibus et de venenis: II.4 [= p. 203]; N.42 [= p. 384].

Dynameron: II.4 [= p. 202]; N.42 [= p. 383].

Iatrica: II.4 [= p. 203]; N.42 [= p. 383].

Physica et Antipathetica: II.4 [= p. 202]; N.42 [= p. 383].

\section{Aëtius Amidenus}

Excerpta varia: II.7 [= p. 207]; N.43 [= p. 385].

Iatricorum libri XVI: II.5 [= p. 203]; N.43 [= p. 384].

\section{Africanus}

De ponderibus et mensuris: II.8 [= p. 209]; N.44 [= p. 385].

Ex Africani Cestis cestum XIII cap. 22: De purgantibus simplicibus: II.8 [= p. 209];

\section{Aglaias}

$$
\text { N.43 [= p. 385]. }
$$

Ad suffusiones incipientes Aglaiae ..., Alexandri discipuli, condiscipuli Demosthenis et amici, versus heroelegi: II.8 [= p. 210]; N.44 [= p. 386].

\section{Alexander}

Hippocratis vel Alexandri epistula ad Ptolemaeum regem: II.8 [= p. 210].

\section{Alexander Aphrodisiensis}

De febribus: II.10 [= p. 212].

De mixtione: II.10 [= p. 212].

De nutritione et augmento: II.9 [= p. 212].

Excerpta varia: II.10 [= p. 213].

Problematum physicorum et medicorum eclogae. Libri V: II.9 [= p. 210]; N.44 [= p. 386].

Quarundam difficultatum solutiones: II.9 [= p. 211]; N.44 [= p. 386].

\section{Alexander Rex}

De septem herbarum facultate: II.10 [= p. 213]; N.44 [= p. 386].

\section{Alexander Sophista}

De hominis generatione: II.11 [= p. 214]. 
De sacrarum herbarum temperamentis: II.11 [= p. 213].

\section{Alexander Trallianus}

De oculis. Libri III: II.12 [= p. 215].

Epistula de lumbricis: II.12 [= p. 215]; N.45 [= p. 387].

Excerpta varia: II.13 [= p. 216].

Therapentica. Libri XII: II.11 [= p. 214].

Alexander (Trallianus?)

Opusculum de pulsunm dignotione in febricitantibus et aphorismi de urinis: II.13 [= p. 216].

Andreas Caesareae In Cappadocia Archiepiscop[us]

Interrogationes et responsiones ex libro II. therapenticae: II.14 [= p. 216].

Andreiomenus

Notae medicae: II.14 [= p. 217].

\section{Andromachus}

Excerpta: II.14 [= p. 217] .

Theriaca ex anguibus, Tranquillitas dicta: II.14 [= p. 217]; N.45 [= p. 387].

Antonius Pyropulus

Excerpta varia: II.15 [= p. 217]; N.45 [= p. 387].

Antyllus

Excerpta varia: II.15 [= p. 218].

Apollonius Citiensis

Commentarius in Hipp[ocratis] de articulis, libri III: II.15 [= p. 218].

Archelaus

Fragmentum: II.16 [= p. 219].

Archigenes

Excerpta varia: II.16 [= p. 219].

Aretaeus

De causis et signis acutorum morborum libri II: II.17 [= p. 220]; N.45 [= p. 387].

De causis et signis diuturnorum morborum libri II: II.17 [= p. 221]; N.45 [= p. 387].

De curatione acutorum morborum libri II: II.18 [= p. 222]; N.45 [= p. 387].

De curatione diuturnorum morborum libri II: II.18 [= p. 223]; N.45 [= p. 387].

\section{Artemidorus}

De urinis: II.19 [= p. 224].

\section{Asclepiades}

Excerpta varia: II.20 [= p. 225].

Praecepta salubria: II.20 [= p. 225]; N.46 [= p. 388].

\section{Athenaeus}

De remediis ordine alphabetico: II.21 [= p. 226].

Excerpta: II.21 [= p. 227].

Synopsis de urinis: II.20 [= p. 226]. 


\section{Basilius (S[anctus])}

Ad Eustatbium medicum de obiectionum haereticorum solutione: II.21 [= p. 227];

N.46 [= p. 388].

De hominis generatione. Libri III: II.21 [= p. 227].

\section{Beniaminus}

Excerpta: II.22 [= p. 228]; N.46 [= p. 388].

Medicamenta purgantia omne cerebri ac stomachi excrementum: II.22 [= p. 228].

Medicinae variae Beniamini Iudaei: II.22 [= p. 227].

\section{Cassius Iatrosophista}

Quaestiones medicae et problemata physica: II.22 [= p. 228]; N.46 [= p. 388].

\section{Cornelius Celsus}

Fragmentum: II.23 [= p. 229].

\section{Chariton}

De pastillis, pillulis, zulapiis et clysteribus: II.23 [= p. 229].

Chrysostomius (?)/Chrysostomus (sic!) (see Ioannes Chrysostomus = II.52 [= p. 266] and N.46 [= p. 389])

De corporis humani constitutione demonstratio: II.23 [= p. 229]; N.46 [= p. 389].

\section{Cleopatra}

De mensuris et ponderibus: II.24 [= p. 229]; N.47 [= p. 389].

Excerpta varia: II.24 [= p. 230].

\section{Coeranus}

De arte recuratoria: II. 24 [= p. 230].

\section{Constantinus Melitiniota}

Antidotarium e persica lingua in graecam a Constantino Melitiniota versum: II.24

$$
\text { [= p. 230]; N.47 [= p. 389]. }
$$

Constantinus Taronites (see N.67 [= p. 418]: Taronitus [?])

Excerpta: N.47 [= p. 389].

\section{Crateuas}

De herbis: II.25 [= p. 231]; N.47 [= p. 389].

\section{Craton}

Prognostica de infirmorum vita et morte: II.25 [= p. 231].

\section{Cyrillus Alexandrinus}

De mensuris et ponderibus: II.25 [= p. 231]; N.47 [= p. 390].

Excerpta: II.25 [= p. 231].

\section{Damascenus}

Excerpta: II.25 [= p. 232].

\section{Damascius}

Expositio in aphorismos Hippocratis. Sectiones VII: II.26 [= p. 232].

Expositio in Prognostica Hippocratis. Sectiones II: II.26 [= p. 232].

\section{Damnastes}

De curatione puerperarum et infantium: II.26 [= p. 232]; N.47 [= p. 390]. 
Excerpta: II.26 [= p. 233].

\section{Democritus}

De sympathicis et antipatbicis: II. 26 [= p. 233]; N.48 [= p. 390].

Excerpta varia: II.27 [= p. 233]; N.48 [= p. 390].

Liber V ad Leucippum: II.115 [= p. 353].

Physica et mystica: II.115 [= p. 353].

Prognostica: II.27 [= p. 233].

\section{Diocles}

Ad Antigonum regem epistula de mensuris et ponderibus: II.28 [= p. 235].

Ad Antigonum regem epistula de tuenda valetudine: II.27 [= p. 234]; N.48 [= p. 390].

Excerpta varia: II.29 [= p. 235].

Hippocratis vel, ut alii volunt, Dioclis epistula ad Ptolemaeum Aegypti regem: II.28 [= p. 235].

\section{Diodorus}

De mensuris et ponderibus: II.29 [= p. 236].

\section{Dionysius Simplex}

De fabrica bominis: II.29 [= p. 236].

\section{Dioscurides}

De facile parabilibus tam simplicibus quam compositis medicamentis libri II: II.32 [= p. 241]; N.49 [= p. 392].

De iis, quae virus eiaculantur, animalibus libellus, in quo et de rabioso cane: II.32

[= p. 240]; N.49 [= p. 392].

De materia medica libri $V$ : II.29 [= p. 236]; N.48 [= p. 391].

De mensuris et ponderibus: II.33 [= p. 243].

De venenis eorumque praecautione et medicatione: II.31 [= p. 239]; N.49 [= p. 391].

Excerpta/Excerpta varia: II.34 [= p. 243]; N.49 [= p. 393].

Liber agens de experientia medica, de simplicibus et compositis medicamentis, ordine litterarum digestus: II.33 [= p. 242].

Metaphrasis in Nicandri Theriaca et Alexipharmaca: II.33 [= p. 242].

Opera varia: II.29 [= p. 236]; N.48 [= p. 391].

\section{Eleutherus}

Excerpta: II.35 [= p. 245].

\section{Epiphanius}

De duodecim gemmis panno sacerdotis infibulatis: II.36 [= p. 247]; N.50 [= p. 394].

De mensuris et ponderibus: II.36 [= p. 246]; N.50 [= p. 393].

Excerpta varia: II.36 [= p. 247].

Physiologus: II.35 [= p. 245]; N.50 [= p. 393].

\section{Erasistratus}

Excerpta varia: II.36 [= p. 247]. 


\section{Erotianus}

Vocum Hippocraticarum conlectio: II.37 [= p. 248]; N.50 [= p. 394].

\section{Esdras Propheta}

Antidotus Esdrae: II.38 [= p. 249].

De diebus criticis: II.37 [= p. 249]; N.50 [= p. 394].

Pro bydropicis et omnibus morbis frigidis: II.37 [= p. 249]; N.51 [= p. 394].

\section{Euphemius Siculus}

Medicamentorum compositiones ab Euph[emius] S[iculus] et Philippo Nero Rheginensi collectae: II.38 [= p. 250]; N.51 [= p. 395].

\section{Eusebius}

De mensuris et ponderibus: II.38 [= p. 250].

\section{Eustathius}

Excerpta: II.38 [= p. 250].

\section{Eutecnius}

Paraphrasis in Dionysii Ixieutica. Libri II: II.38 [= p. 250].

Paraphrasis in Nicandri Alexipharmaca: II.39 [= p. 251].

Paraphrasis in Nicandri Theriaca: II.39 [= p. 251].

Paraphrasis in Oppiani Halieutica: II.39 [= p. 251].

\section{Galenos}

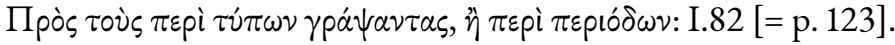

Ad Gaurum quomodo animetur fetus: I.120 [= p. 173].

Ad Glauconem de medendi methodo libri II: I.93 [= p. 135]; N.34 [= p. 372].

Adversus ea quae Juliano in Hippocratis aphorismos enuntiata sunt libellus: I.106

$$
\text { [= p. 153]. }
$$

Adversus Lycum: I.106 [= p. 152].

An animal sit quod est in utero: $\mathrm{I} .110$ [= p. 158]; N.36 [= p. 375].

An in arteriis natura sanguis contineatur: I.71 [= p. 107].

Ars medica: I.61 [= p. 96]; N.30 [= p. 367].

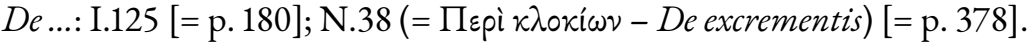

De abortivo foetu: I.120 [= p. 173].

De affectuum renibus insidentium dignotione et curatione liber: I.113 [= p. 165].

De alimentorum facultatibus libri III: I.76 [= p. 114]; N.31 [= p. 369].

De anatomia: I.115 [= p. 168].

De anatomia vivorum: I.116 [= p. 168]; N.37 [= p. 377].

De anatomicis administrationibus libri IX: I.66 [= p. 100].

De angina: I.125 [= p. 180].

De animalibus: I.121 [= p. 175].

De animalibus noxiis: I.121 [= p. 175].

De anni temporibus: I.136 [= p. 192].

De antidotis libri II: I.99 [= p. 142].

De aquis: I.132 [= p. 188]. 
[De arte medica]: I.122 [= p. 176].

De atra bile: $\mathrm{I} .73[=\mathrm{p} .110]$.

De bono habitu: I.71 [= p. 108].

De captionibus penes dictionem: I.100 [= p. 144].

De carbunculis: I.116 [= p. 169].

De causis pulsuum libri IV: I.88 [= p. 129].

De causis respirationis: $\mathrm{I} .70$ [= p. 105$]$.

De cerebro eiusque tunicis: I.119 [= p. 172].

De chirurgia: I.135 [= p. 191].

De chirurgorum operationibus et de decubitu infirmorum: I.135 [= p. 191].

De comate secundum Hippocratem: I.83 [= p. 124].

De compositione medicamentorum per genera libri VII: I.98 [= p. 142].

De compositione medicamentorum secundum locos libri X: I.97 [= p. 141]; N.34 $[=$ p. 372].

De constitutione artis medicae ad Patropbilum liber: I.60 [= p. 96]; N.30 [= p. 367].

De consuetudinibus: I.119 [= p. 172].

De contusionibus: I.122 [= p. 176].

De corporis partibus: $\mathrm{I} .126$ [= p. 181]; N.38 [= p. 378].

De crisibus libri III: I.89 [= p. 132]; N.33 [= p. 371].

De cruditate: $\mathrm{I} .117$ [= p. 169].

De cuiuslibet animi peccatorum dignotione atque medela: I.73 [= p. 110].

De curandi ratione per venae sectionem: I.94 [= p. 137].

De demonstrationibus: I.117 [= p. 169].

De diaeta et morbis curandis: I.118 [= p. 171]; N.37 [= p. 377].

De diebus decretoriis libri III: I.90 [= p. 132].

De differentiisfebrium libri II: I.80 [= p. 120]; N.32 [= p. 371].

De differentiis morborum: $\mathrm{I} .78$ [= p. 116].

De difficultate respirationis libri III: I.84 [= p. 126].

De dignoscendis pulsibus libri IV: I.87 [= p. 129]; N.33 [= p. 371].

De dignotione ex insomniis: I.77 [= p. 116].

De dignotione hominum purgandorum: I.124 [= p. 178].

De dolore capitis: I.125 [= p. 180].

De elementis: I.131 [= p. 186].

De elementis secundum Hippocratem: I.63 [= p. 98].

De erysipelate: $\mathrm{I} \cdot 121[=\mathrm{p} .174]$.

De excrementis: N.38 [= p. 378] (I.125 [= p. 180] “De ...”).

De facultatibus naturalibus: I.65 [= p. 100]; N.30 [= p. 368].

De fasciis: I.107 [= p. 153].

De febribus: I.130 [= p. 186].

De foetuum formatione: $\mathrm{I} .70$ [= p. 107].

De generatione: $\mathrm{I} .117$ [= p. 170]. 
De gynaeceis i. e. de passionibus mulierum: I.118 [= p. 170].

De hirudinibus, revulsione, cucurbitula, incisione et scarificatione: I.95 [= p. 137].

De historia philosopha: I.110 [= p. 159].

[De hominis natura]: I.135 [= p. 191]; N.39 [= p. 380].

De hominis natura testamentum: I.124 [= p. 179].

De bumoribus: I.136 [= p. 192].

De bumoribus (K. XIX 485-496): I.111 [= p. 161].

De iecore: $\mathrm{I} .122$ [= p. 175$]$.

De iis, quae medice scripta sunt in Platonis Timaeo, fragmentum: I.129 [= p. 185].

De inaequali intemperie: I.83 [= p. 125].

De insomniis: I.120 [= p. 174].

De instrumento odoratus: I.68 [= p. 103].

De lacte: I.117 [= p. 170].

De lapidibus: I.126 [= p. 181]; N.38 [= p. 378].

De laterum morbo ad Patrophilum: I.129 [= p.185].

De lepra alba: I.126 [= p. 181].

De libris propriis: I.109 [= p. 156].

De locis affectis libri VI: I.85 [= p. 126].

De machinamentis: I.126 [= p. 181].

De marcore: I.83 [= p. 124]; N.32 [= p. 371].

De materia medica: I.132 [= p. 188].

De medicis: I.123 [= p. 177].

De melancholia ex Galeno, Rufo, Posidonio et Marcello Sicamii Aetii libellus: I.114 [= p. 165].

De metallis: $\mathrm{I} .126$ [= p. 181].

De morbis Excerpta: I.126 [= p. 181]; N.38 [= p. 378].

De morborum causis: I.78 [= p. 117]; N.32 [= p. 370].

De morborum temporibus: I.81 [= p. 121].

De motu musculorum libri II: I.69 [= p. 105].

De musculorum dissectione: I.108 [= p. 155].

De nervorum dissectione: I.67 [= p. 102].

De nimia seminis profusione: I.117 [= p. 170].

De non intentis: I.116 [= p. 168].

De oculis: $\mathrm{I} .129$ [= p. 184].

De optima corporis nostri constitutione: I.71 [= p. 107]; N.30 [= p. 368].

De optima doctrina: I.59 [= p. 94].

De optima secta ad Thrasybulum liber: I.60 [= p. 95].

De optima ventris concoctione: I.129 [= p. 184].

De ordine librorum suorum ad Eugenianum: I.109 [= p. 157].

De ossibus ad tirones: I.67 [= p. 101]; N.30 [= p. 368].

De paroxysmorum temporibus: I.129 [= p. 184]. 
De partibus philosophiae: $\mathrm{I} .134$ [= p. 190].

De parvae pilae exercitio: I.74 [= p. 113]; N.31 [= p. 369].

De placitis Hippocratis et Platonis libri IX: I.74 [= p. 112]; N.31 [= p. 369].

De plenitudine: I.82 [= p. 123].

De podagra: I.130 [= p. 185]; N.39 [= p. 379].

De ponderibus et mensuris: I.115 [= p. 167].

De praegnotione ad Epigenem liber: I.100 [= p. 145].

De praenotione: $\mathrm{I} .112$ [= p. 161].

De praesagitione ex pulsibus libri IV: I.88 [= p. 130].

De probis pravisque alimentorum sucis: I.77 [= p. 115]; N.32 [= p. 370].

De propriis placitis: I.119 [= p. 172].

De propriorum animi cuiuslibet affectuum dignotione et curatione: I.72 [= p. 109]; N.31 [= p. 369].

Deptisana: I.77 [= p. 115].

De pulsibus: I.131 [= p. 187].

De pulsibus ad Antonium: I.113 [= p. 164]; N.37 [= p. 376].

De pulsibus ad tirones: I.86 [= p. 127]; N.33 [= p. 371].

De pulsuum differentiis libri IV: I.87 [= p. 128]; N.33 [= p. 371].

De purgantium medicamentorum facultate: I.95 [= p. 137].

De quinque sensibus: I.115 [= p. 167 ].

De remediis parabilibus libri III: I.99 [= p. 144]; N.34 [= p. 372].

De respirationis usu: $\mathrm{I} .70$ [= p. 106].

De sanitate tuenda libri VI: I.75 [= p. 113]; N.31 [= p. 369].

De sectis ad eos qui introducuntur: I.60 [= p. 95]; N.29 [= p. 367].

De semine libri II: I.70 [= p. 106].

De septimestri partu: $\mathrm{I} .121$ [= p. 174].

De simplicium medicamentorum temperamentis et facultatibus libri XI: I.96 [= p. 139]; N.34 [= p. 372].

De spermate: $\mathrm{I} .131$ [= p. 186].

De stomacho: $\mathrm{I} .131$ [= p. 187$]$.

De substantia facultatum naturalium fragmentum: I.72 [= p. 108].

De succedaneis liber: I.114 [= p. 166]; N.37 [= p. 377].

De symptomatum causis libri III: I.79 [= p. 119]; N.32 [= p. 370].

De symptomatum differentiis: $\mathrm{I} .79$ [= p. 118]; N.32 [= p. 370].

De temperamentis: I.64 [= p. 99].

De temporibus etesiarum et quomodo iis utendum sit: N.37 [= p. 377].

De theriaca: I.122 [= p. 176].

De theriaca ad Pamphilianum: I.99 [= p. 143].

De theriaca ad Pisonem liber: I.99 [= p. 143].

De totius morbi temporibus: I.81 [= p. 122].

De tremore, palpitatione, convulsione et rigore: I.82 [= p. 123]. 
De tumoribus praeter naturam: I.83 [= p. 125].

De typis: I.82 [= p. 122].

De unguentis: I.115 [= p. 168].

De urinis: I.127 [= p. 182]; N.39 [= p. 379].

De urinis (K. XIX 574-601): I.113 [= p. 163]; N.37 [= p. 376].

De urinis compendium: $\mathrm{I} .113$ [= p. 164].

De urinis ex Hippocrate, Galeno aliisque quibusdam: I.113 [= p. 164]; N.37 [= p. 376].

De usu partium corporis humani libri XVII: I.68 [= p. 104]; N.30 [= p. 368].

De usu pulsuum: I.73 [= p. 111].

De uteri dissectione: $\mathrm{I} .68[=\mathrm{p} .103]$.

De vasis vitreis: $\mathrm{I} .132$ [= p. 187].

De venae sectione: $\mathrm{I} .134$ [= p. 190]; N.39 [= p. 380].

De venae sectione (K. XIX 519-528): I.112 [= p. 162].

De venae sectione adversus Erasistrateos Romae degentes: I.94 [= p. 136].

De venae sectione adversus Erasistratum: I.94 [= p. 136].

De venarum arteriarumque dissectione: $\mathrm{I} .67[=\mathrm{p} .102]$.

De venereis: I.75 [= p. 113].

De ventis, igne, aquis, terra: I.116 [= p. 168].

De victu attenuante: $\mathrm{I} .125$ [= p. 180].

De victu singulis mensibus servando: I.124 [= p. 179].

De victus ratione: $\mathrm{I} .118$ [= p. 171]; N.37 [= p. 377].

De victus ratione in morbis acutis ex Hippocratis sententia: $\mathrm{I} .110$ [= p. 158].

De vita et morte: $\mathrm{I} .121[=$ p. 175$]$.

De vulneribus: $\mathrm{I} .120$ [= p. 173].

De XII portis: I.122 [= p. 176].

Definitiones medicae: I.111 [= p. 159]; N.36 [= p. 375].

Distinctio: I.118 [= p. 171].

Excerpta varia: $\mathrm{I} .148$ [= p. 192]; N.41 [= p. 380].

Fauces definiuntur: I.127 [= p. 182].

Galeni et Simplicii testimonia de Epicteto: I.120 [= p. 174].

Galeni in Hippocratis de natura hominis lib[rum] commentarii II: I.101 [= p. 146]; N.34 [= p. 373].

Galeni paraphrastae Menodoti suasoria ad artes oratio: I.59 [= p. 93].

Hippiatrosophium: I.123 [= p. 178].

Hippocratis aphorismi et Galeni in eos commentarii VII: I.104 [= p. 151]; N.35 [= p. 374].

Hippocratis liber resolutionis, quem Galenus explicat: I.124 [= p. 178]; N.38 [= p. 378].

Iatrosophia: I.123 [= p. 176]; N.38 [= p. 377].

In Hippocratis de articulis librum commentarii IV: I.106 [= p. 153]. 
In Hippocratis de bumoribus librum commentarii III: I.103 [= p. 148]; N.35 [= p. 374].

In Hippocratis epidemiarum librum primum commentarii III: I.104 [= p. 150]; N.35 [= p. 374].

In [Hippocratis epidemiarum] librum secundum commentarii V: I.104 [= p. 150]; N.35 [= p. 374].

In [Hippocratis epidemiarum] librum sextum commentarii VI: I.104 [= p. 150]; N.35 [= p. 374].

In [Hippocratis epidemiarum] librum tertium commentarii III: I.104 [= p. 150]; N.35 [= p. 374].

In Hippocratis librum de acutorum victu commentarii IV: I.102 [= p. 148]; N.35 [= p. 373].

In Hippocratis librum de alimento commentarii IV: I.102 [= p. 147].

In Hippocratis librum de fracturis commentarii III: I.108 [= p. 155].

In Hippocratis librum de officina medici commentarii III: I.108 [= p. 155].

In Hippocratis praedictionum librum primum commentarii III: I.103 [= p. 149].

In Hippocratis prognosticum commentarii III: I.107 [= p. 154]; N.35 [= p. 374].

In Hippocratis vel Polybi opus de salubri victus ratione privatorum commentarius:

I.101 [= p. 147]; N.34 [= p. 373].

In libr[um] Hippocratis de aetatum aegritudinibus: I.124 [= p. 178].

Indices in Galenum: I.150 [= p. 194].

Institutio logica: I.119 [= p. 171].

Introductio sive medicus: I.100 [= p. 145]; N.34 [= p. 373].

Lexicon botanicum: $\mathrm{I} .117$ [= p. 169].

Linguarum s[ive] dictionum exoletarum Hippocratis explicatio: I.109 [= p. 157];

N.36 [= p. 374].

Methodi medendi libri XIV: I.91 [= p. 133]; N.33 [= p. 371].

Opera varia: I.58 [= p. 93]; N.29 [= p. 367].

Praesagitio omnino vera expertaque: I.112 [= p. 162].

Prognostica: $\mathrm{I} .130$ [= p. 185]; N.39 [= p. 379].

Prognostica de decubitu ex mathematica scientia: I.112 [= p. 162]; N.36 [= p. 376].

Puero epileptico consilium: I.96 [= p. 138].

Qua ratione adiuvandi ii sint, qui remedio hausto non purgantur: I.124 [= p. 179].

Quod animi mores corporis temperamenta sequantur: $\mathrm{I} .72[=$ p. 109]; N.31 [= p. 368].

Quod optimus medicus sit etiam philosophus: I.59 [= p. 94].

Quod qualitates incorporeae sint: I.111 [= p. 160]; N.36 [= p. 375].

Quomodo morbum simulantes sint deprehendendi: I.109 [= p. 156].

Quos, quibus catharticis medicamentis et quando purgare oporteat: I.95 [= p. 138].

Remedia: I.132 [= p. 188]; N.39 [= p. 379].

Scholia in Galenum: I.150 [= p. 194].

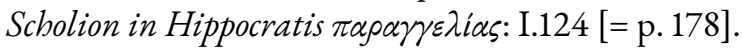


Sermo adversus empiricos medicos: I.120 [= p. 173].

Synopsis librorum suorum sedecim de pulsibus: I.89 [= p. 131]; N.33 [= p. 371].

Thrasybulus sive utrum medicinae sit an gymnastices bygieine: I.74 [= p. 112].

\section{Gregorius Nyssenus}

De hominis opificio: II.39 [= p. 252]; N.51 [= p. 395] (see Nemesius Emesenus = II.66 [= p. 283])

De pulsibus: II.40 [= p. 253].

Excerpta varia: II.40 [= p. 254].

Oratio adversus astronomos: II.40 [= p. 253].

\section{Gregorius Theologus}

Alatium: II.41 [= p. 254]; N.51 [= p. 395] (see Lucas = II.58 [= p. 272] and N.56 $[=\mathrm{p} .402])$.

De compositione et distinctione corporis humani: II.40 [= p. 254].

\section{Harpocration}

De facultatibus naturalibus animalium et herbarum et lapidum: II.41 [= p. 255].

Epistula ad imperatorem: II.41 [= p. 255].

Excerpta varia: N.51 [= p. 396].

\section{Heliodorus}

Ad Theodosium regem: II.41 [= p. 255]; N.52 [= p. 396].

Chirurgia: N.52 [= p. 396].

De mensuris et ponderibus: II.42 [= p. 256].

Excerpta varia: II.42 [= p. 256].

\section{Heraclius}

Versus politici: II.43 [= p. 256].

\section{Hermes Trismegistus}

Ad Asclepium de animalium proprietatibus et remediis quae ex illis peti possunt, cum figuris: II.45 [= p. 260].

Ad Asclepium de plantis VII planetarum: II.45 [= p. 260]; N.53 [= p. 399].

Ad Asclepium de plantis XII zodiaci signorum: II.45 [= p. 260]; N.53 [= p. 398].

Ad Asclepium liber universalis: II.44 [= p. 259]; N.53 [= p. 398].

Canon: II.43 [= p. 257].

Canon de decubitu infirmorum: II.44 [= p. 259]; N.53 [= p. 398].

De decanis ad Asclepium: II.45 [= p. 260].

De ponderibus: II.45 [= p. 261].

De succis plantarum: II.44 [= p. 259]; N.53 [= p. 398].

De XII locorum nomenclatura et facultate: II.45 [= p. 260]; N.53 [= p. 399].

Excerpta varia: II.47 [= p. 261]; N.45 [= p. 399].

Iatromathematica ad Ammonium Aegyptium: II.44 [= p. 258]; N.53 [= p. 397].

Kyranides: II.43 [= p. 257]; N.52 [= p. 397].

Methodus ad omne inceptum idonea: II.43 [= p. 257]; N.52 [= p. 397].

Opera varia: II.43 [= p. 257]; N.52 [= p. 397]. 
Organum: II. 43 [= p. 257].

\section{Herodotus}

Diagnostica de morbis acutis et chronicis: II.48 [= p. 262].

\section{Herophilus}

Defebricantibus: II.48 [= p. 262]; N.54 [= p. 399].

Definitiones pulsuum: II.49 [= p. 262].

\section{Hierophilus Sophista}

[Defebricantibus]: N.54 [= p. 400].

De alimentis, quae singulis mensibus sumenda aut vitanda sunt: II.49 [= p. 263].

De alimentorum facultatibus: II.49 [= p. 263].

\section{Hippokrates}

Пepi $\alpha \nu \alpha \tau о \mu \tilde{\eta} \varsigma \tau \tilde{\omega} \nu \zeta \omega \nu \tau \tilde{\omega} v:$ N.27 [= p. 361] (with a reference [Zu Kat. I S. 39] $[=$ p. 73] that does not correspond).

Пвpi $\pi \alpha \theta \tilde{\omega} v:$ N.28 [= p. 363] (with a reference [Zu Kat. I S. 44] [= p. 82-83] that does not correspond).

Ad Ptolemaeum regem epistula de hominis fabrica: I.40 [= p. 75]; N.27 [= p. 361]. Aphorismi: I.12 [= p. 19]; N.25 [= p. 359].

Coa praesagia: $\mathrm{I} .20[=$ p. 31$]$.

De aëre, aquis et locis: I.4 [= p. 9].

De affectionibus (L. VI 208-270): I.23 [= p. 37]; N.26 [= p. 360].

De alimento: I.33 [= p. 62].

De alimentorum differentiis: I.47 [= p. 86].

De aloe: I.39 [= p. 73].

De anatome: $\mathrm{I} .31$ [= p. 58].

De anatomicis ferramentis: I.39 [= p. 73].

De angina: I.43 [= p. 81$]$.

De animalibus marinis et terrestribus ad morbos curandos idoneis: I.42 [= p. 78].

De aquis: $\mathrm{I} .48[=$ p. 86$]$.

De arte: $\mathrm{I} .20$ [= p. 32].

De articulis: I.11 [= p. 17].

De calce: $\mathrm{I} .46$ [= p. 83].

De capitis vulneribus: I.10 [= p. 15].

De carnibus: $\mathrm{I} .32[=$ p. 60].

[Dechirurgia]: I.49 [= p. 88].

De collo vesicae: $\mathrm{I} .43$ [= p. 81].

De corde: I.33 [= p. 62].

De corpore et generatione hominis: I.47 [= p. 86].

De decenti ornatu: I.34 [= p. 65].

De dentitione: I.32 [= p. 59].

De diaeta (regimine) acutorum: I.8 [= p. 12].

De diebus indicatoriis: I.35 [= p. 67]. 
De elementis: I.46 [= p. 84].

De exsectione foetus: $\mathrm{I} .31$ [= p. 57].

De exsectione pueri: I.31 [= p. 57].

De febribus: I.46 [= p. 83].

De febrium differentiis: I.46 [= p. 84].

De fistulis: I.25 [= p. 42].

De flatibus: I.22 [= p. 35].

De fracturis: I.11 [= p. 16].

De generatione hominis: I.39 [= p. 74].

De genitura: I.28 [= p. 50]; N.26 [= p. 360].

De glandulis: I.32 [= p. 59].

De baemorrboïdibus: I.24 [= p. 41].

De humidorum usu: I.22 [= p. 36].

De humoribus: I.19 [= p. 29].

De iecore: I.42 [= p. 78].

De insomniis: I.26 [= p. 44].

De internis affectionibus: I.27 [= p. 46]; N.26 [= p. 360].

De iudicationibus: I.35 [= p. 67].

De IV aetatibus praenotio: I.49 [= p. 88]; N.28 [= p. 363].

De lapidum virtute: I.43 [= p. 81].

De locis in homine: I.23 [= p. 38].

De medico: I.34 [= p. 65].

De mensibus: $\mathrm{I} .43$ [= p. 81$]$.

De morbis I: I.23 [= p. 36]; N.26 [= p. 359].

De morbis II et III: I.26 [= p. 45]; N.26 [= p. 360].

De morbis liber IV: I.29 [= p. 52]; N.26 [= p. 360].

De morbis hominum et remediis: I.44 [= p. 82].

De morbis popularibus I et III: I.10 [= p. 13].

De morbis popularibus II. IV-VII: I.19 [= p. 28].

De morbo sacro: I.24 [= p. 39]; N.26 [= p. 360].

De mulieribus: $\mathrm{I} .39$ [= p. 74].

De mulierum affectibus: I.29 [= p. 53].

De natura hominis: I.21 [= p. 33]; N.26 [= p. 359].

De natura muliebri: I.27 [= p. 47]; N.26 [= p. 360].

De natura pueri: I.29 [= p. 51].

De octimestri partu: I.28 [= p. 49].

De officina medici: I.11 [= p. 15].

De ossium natura: $\mathrm{I} .34$ [= p. 64].

De partibus hominis: I.43 [= p. 81]; N.28 [= p. 362].

De pede: I.44 [= p. 83].

De prisca medicina: I.4 [= p. 8]. 
De pulsibus: I.47 [= p. 85].

De quattuor elementis et humoribus naturae hominis: I.46 [= p. 84]; N.28 [= p. 363].

De remediis: $\mathrm{I} .49$ [= p. 87].

De salubri diaeta: $\mathrm{I} .22$ [= p. 34].

De sensibus: I.39 [= p.73]; N.27 [= p. 361].

De septimanis: I.32 [= p. 60]; N.27 [= p. 361].

De septimestripartu: I.27 [= p. 48].

De specie atrae bilis: $\mathrm{I} .49$ [= p. 88].

De splene et stomacho: $\mathrm{I} .46[=$ p. 84$]$.

De sterilibus: $\mathrm{I} .30$ [= p. 54].

De substantia artis medicae. Definitio artis medicae: I.48 [= p. 87].

De sudoribus: I.42 [= p. 80].

De superfoetatione: I.31 [= p. 56].

De ulceribus: I.24 [= p. 40].

De unguentis: I.39 [= p. 73].

De urinis: I.44 [= p. 82]; N.28 [= p. 362].

De vasis vitreis: $\mathrm{I} .48$ [= p. 86$]$.

De venae sectione: $\mathrm{I} .49$ [= p. 88].

De victus ratione I-III: I.25 [= p. 42].

De virginum morbis: I.30 [= p. 55]; N.26 [= p. 361].

De visu: $\mathrm{I} .34$ [= p. 63].

Decretum Atheniensium: I.36 [= p. 68]; N.27 [= p. 361].

Epistula ad Ptolemaeum regem de sanitate tuenda: I.41 [= p. 76]; N.27 [= p. 362].

Epistulae: I.36 [= p. 68]; N.27 [= p. 361].

Esse re vera artes: $\mathrm{I} .48[=\mathrm{p} .86]$.

Excerpta varia: I.56 [= p. 89]; N.29 [= p. 363].

Hippiatrica: $\mathrm{I} .43[=$ p. 80$]$.

Hippocratis ad Galenum discipulum liber de pulsibus et de temperamentis corporis bumani: I.47 [= p. 85].

Hippocratis ad Ptolemaeum regem epistula: I.41 [= p. 77].

Hippocratis et Galeni philosophia de natura hominis: I.43 [= p. 80].

Hippocratis vel Dioclis epistula ad Ptolemaeum Aegypti regem: I.40 [= p. 74].

Indices et Lexica: I.57 [= p. 90].

Iusiurandum: I.17 [= p. 25].

Jatrosophium: I.42 [= p. 79]; N.28 [= p. 362].

Laterculi antiqui: $\mathrm{I} .57[=$ p. 90$]$.

Lex: I.18 [= p. 26].

Lexicon specierum (?): I.40 [= p. 74] .

[Medendi methodus]: I.42 [= p. 79].

Opera varia: I.3 [= p. 7]; N.25 [= p. 359]. 
Oratio ad aram: $\mathrm{I} .36[=$ p. 68$] ;$ N.27 [= p. 361].

Praeceptiones: I.35 [= p. 66].

Praesagiorum liber I: I.20 [= p. 30].

Praesagiorum liber II: I.33 [= p. 61].

Prognostica: $1.44[=$ p. 83$] ;$ N.28 [= p. 363].

Prognosticon: I.5 [= p. 9]; N.25 [= p. 359].

Qualem oporteat esse discipulum: I.43 [= p. 81].

Remedia: I.48 [= p. 87 ]; N.28 [= p. 363].

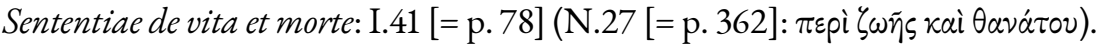

Testamentum: $\mathrm{I} .39[=$ p. 74$]$.

Thessali legati oratio: $1.36[=$ p. 68$] ;$ N.27 [= p. 361].

Vectiarius: I.12 [= p. 18].

\section{Hypatus}

Interpretatio partium corporis: II.50 [= p. 264].

\section{Iacobus Psychrestus}

Excerpta: II.50 [= p. 264].

\section{Ioannes Alexandrinus (nicht Philoponus)}

Scholia in Hippocratis librum de natura pueri ab ore Ioannis: II.51 [= p. 265].

\section{Ioannes Antiochenus Archiatrus}

Collectio remediorum ex Dioscoride, Hippocrate et Galeno: II.51 [= p. 265].

\section{Ioannes Archiatrus}

Variorum morborum curatio: II.52 [= p. 266]; N.54 [= p. 400].

Ioannes Chrysostomus (N.54 [= p. 400]: Vgl. Nachtrag über Chrysostomus = N.46 [= p. 389])

De adversa valetudine et medicis: II.52 [= p. 266].

\section{Ioannes Chumnos}

Quali victus ratione podagra evitari possit: II.52 [= p. 267].

\section{Ioannes Damascenus}

Excerpta: II.53 [= p. 267].

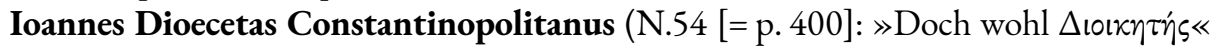
Treu)

De medicina: II.53 [= p. 267]; N.54 [= p. 400].

\section{Ioannes Episcopus Prisdrianensis}

Collectanea de excrementis: II.54 [= p. 268]; N.55 [= p. 400].

Collectanea de intestinis, ex Palladio, Archelao, Stephano Alexandrino etc.: II.55

$$
\text { [= p. 269]; N.55 [= p. 401]. }
$$

Collectanea de urinis: II.55 [= p. 268]; N.55 [= p. 401].

\section{Ioannes Esdra}

De urinis: II.53 [= p. 267].

\section{Ioannes Iatrosophista}

De morbis eorumque curationibus: II.54 [= p. 268]. 


\section{Ioannes Philoponus Alexandrinus}

De febribus: II.50 [= p. 265].

De pulsibus: II.50 [= p. 265].

\section{Ioannes Pothus Pediasimus}

( $V g l$ Krumbacher, Sitz. Ber. d. Münch. Akad. d. Wiss. 1892 p. 350): II.54 [= p. 268].

\section{Ioannes Spensatus}

Liber de incurabilibus et difficile curabilibus morbis: II.55 [= p. 269].

\section{Ioannes Staphidaces}

De nonnullorum medicamentorum compositione: II.55 [= p. 269]; N.55 [= p. 401].

Ioannes Zacharias Actuarius (S. unter Zacharias)

\section{Iustus}

Excerpta: II.56 [= p. 270]; N.56 [= p. 401].

\section{Karastonus}

Liber de mensuris et ponderibus, editus a Thebit filio Core: II.56 [= p. 270].

Kyranus ( $\mathrm{g} g$. Harpocration und Hermes Trismegistus)

Lexicon de lapidibus, plantis etc.: II.56 [= p. 270].

Opus physico-magicum de naturalibus virtutibus: II.56 [= p. 270].

\section{Lampudes}

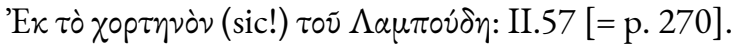

Leo

De hominum proprietatibus: II.57 [= p. 271].

Excerpta: II.57 [= p. 271].

Synopsis artis medicae: II.57 [= p. 271]; N.56 [= p. 401].

Synopsis de hominis natura: II.57 [= p. 271].

\section{Libanius}

De hominis generatione: II. 57 [= p. 272].

\section{Logadius}

Excerpta: II.58 [= p. 272]; N.56 [= p. 402].

Lucas ( Vgl. Gregorius Theol[ogus] = II.41 [= p. 254])

Compositio salsi intinctus: II.58 [= p. 272]; N.56 [= p. 402].

Excerpta varia: II.58 [= p. 273]; N.56 [= p. 402].

\section{Lycus}

De vulva et muliebri pudendo: II.59 [= p. 273].

Excerpta varia: II.59 [= p. 273].

\section{Macarius Magnes}

De urinis: II.59 [= p. 273].

Macarius Maximus (N.56 [= p. 403]: d.i. uakápıos (beatus) Maximus, scil[icet]

Confessor)

De anima: II.59 [= p. 274]; N.56 [= p. 403]. 


\section{Magnus Emesenus}

De febribus: II.60 [= p. 274].

De urinis ex ore Theophili: II.59 [= p. 274]; N.57 [= p. 403].

Excerpta: II.60 [= p. 275].

Prognostica: II.59 [= p. 274].

Mangens (?) (= Macarius Magnes oder Magnus Emesenus?)

Synopsis de urinis: II.60 [= p. 275].

\section{Manuel Comnenus}

De compositione medicamentorum: N.57 [= p. 403] (with a reference to II.60 $[=$ p. 274] that does not correspond).

\section{Marcellinus}

Depulsibus: II.60 [= p. 275]; N.57 [= p. 403].

\section{Marcellus Sidetes}

De mensuris et ponderibus: II.61 [= p. 276].

Medicina ex piscibus: II.61 [= p. 275].

\section{Marcianus}

Medicamentum ad nervos laxandos idoneum: II.61 [= p. 276].

\section{Marianus}

Excerpta: II.62 [= p. 276]; N.57 [= p. 404].

Maximus (Planudes?) (N.57 [= p. 404]. Vgl. Nachtrag zu Meletius Excerpta varia (N.59 [= p. 406])

De sanguinibus: II.62 [= p. 276].

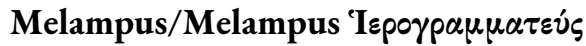

De naevis corporis: N.48 [= p. 405].

De palpitationibus: II.62 [= p. 277]. See below.

Expalpitatione divinatio ad regem Ptolemaeum: N.58 [= p. 404]. See above.

\section{Meletius Monachus}

Commentarius in Hippocratis aphorismos: II.63 [= p. 279].

De anima: II.62 [= p. 277]; N.58 [= p. 405].

De natura hominis: II.63 [= p. 277]; N.58 [= p. 405].

De quattuor elementis: II.63 [= p. 277].

Excerpta varia: II.64 [= p. 279]; N.59 [= p. 406].

\section{Menander Iatrosophista}

De mulieribus: II.64 [= p. 280].

\section{Mercurius Monachus}

De pulsibus: II.64 [= p. 280]; N.59 [= p. 406].

\section{Methodius Causocalybitus}

De diaeta hominis: II.65 [= p. 281].

Methodius Patriarchus (N.59 [= p. 406]: d. i. »Patriarch «, nämlich von Konstantinopel, »also zu schreiben Patriarcha « Treu)

Excerpta varia: II.65 [= p. 281]. 


\section{Metrodora}

De mulierurn morbis uteri: II.65 [= p. 282].

\section{Mnesitheus Cyzicenus}

Excerpta varia: II.65 [= p. 282].

\section{Moschion}

Praecepta vitae: II.66 [= p. 282].

\section{Muscio}

De morbis mulierum: II.66 [= p. 282].

Nemesius Emesenus ( $\mathrm{gl}$. Gregorius Nyssenus = II.39 [= p. 252])

De natura hominis: II.66 [= p. 283]; N.59 [= p. 406].

\section{Neophytus Prodromenus}

De dentium doloribus: II.68 [= p. 286].

Remediorum collectio alphabetica: II.68 [= p. 286]; N.60 [= p. 407].

\section{Nepualius}

De sympathicis et antipathicis: II.68 [= p. 287]; N.60 [= p. 407].

\section{Nicolaus Myrepsus}

De compositione medicamentorum, alphabetice: II.69 [= p. 287]; N.60 [= p. 407].

De emplastris: II.69 [= p. 287].

Excerpta: II.69 [= p. 288].

\section{Nicomedes}

Versus in Hippocratem: II.69 [= p. 288].

\section{Nicomedes Iatrosophista}

Lexicon medicum: II.69 [= p. 288].

\section{Ocianus}

De compositione medicamentorum liber $V:$ II.70 [= p. 288].

\section{Olymnius Alexandrinus}

De decubitu: II.70 [= p. 289].

Excerpta: II.70 [= p. 289].

\section{Oribasius}

Ad Eunapium de parabilibus medicamentis. Libri IV: II.72 [= p. 292].

Conspectus ad Eustathium filium. Libri IX: II.71 [= p. 291]; N.61 [= p. 408].

Excerpta varia: II.73 [= p. 293]; N.61 [= p. 408].

Medicae collections ad Iulianum: II.70 [= p. 289].

Opera varia: II.70 [= p. 289]; N.60 [= p. 408].

\section{Orpheus}

De lapidibus: II.75 [= p. 294].

\section{Palladius}

De cibo et potione: II.75 [= p. 296]; N.61 [= p. 409].

Excerpta varia: II.76 [= p. 297].

In Galeni librum de sectis scholia: II.76 [= p. 297].

Scholia in Hippocratis de fracturis: II.76 [= p. 296]; N.61 [= p. 409]. 
Scholia in Hippocratis de popularibus morbis librum sextum, sectiones VIII: II.76 [= p. 296]; N.61 [= p. 409].

Stephani philosophi vel Palladii synopsis de febribus: II.75 [= p. 295]; N.61 [= p. 409].

\section{Pamphilus}

Excerpta: II.76 [= p. 297] (N.62 [= p. 409]: »Nur in Receptensammlungen« Treu).

\section{Pancharius}

Compendium de decubitu infirmorum: II.76 [= p. 297]; N.62 [= p. 410].

Excerpta: II.77 [= p. 297].

\section{Paulus Aegineta}

De mensuris et ponderibus: II.79 [= p. 302]; N.62 [= p. 411].

De succedaneis, ex Galeno: II.78 [= p. 300]; N.62 [= p. 410].

De urinis. Libri VII: II.79 [= p. 302].

De venenis: II.78 [= p. 300].

Epitomae medicae libri septem: II.77 [= p. 298]; N.62 [= p. 410].

Excerpta varia: II.79 [= p. 303]; N.62 [= p. 411].

\section{Paulus Italus}

Excerpta: II. 81 [= p. 305].

\section{Paulus Nicaeensis}

De cognitione et curatione variorum morborum: II.81 [= p. 305]; N.63 [= p. 411].

\section{Perzoe}

Liber physiologicus ex India translatus et Chosroe regi Persarum traditus a Perzoe

\section{Petosiris} etc.: II.81 [= p. 306].

De decubitu infirmorum: II.82 [= p. 307].

Epistula ad regem Nechepso: II.82 [= p. 306]; N.63 [= p. 411].

Excerpta: II.82 [= p. 307].

Organum de vita et morte: II.82 [= p. 307].

\section{Philagrius}

Excerpta varia: II. 83 [= p. 308].

\section{Philaretus}

De pulsuum scientia: II.83 [= p. 308]; N.63 [= p. 412].

\section{Philippus Xerus}

\section{Philo}

Compositiones medicamentorum: N.63 [= p. 412].

Excerpta varia: II.85 [= p. 308]; N.63 [= p. 412].

\section{Philo, Herennius}

De aetatibus hominis: II.85 [= p. 309].

\section{Philumenus}

[De animalibus noxiis et remedis ad ea]: N.64 [= p. 412].

Excerpta varia: II.85 [= p. 309]. 


\section{Photius Monachus}

Carmina: II.85 [= p. 309].

Excerpta: II.86 [= p. 309].

\section{Porphyrius}

Excerpta varia: II.86 [= p. 310].

\section{Posidonius}

\section{Proclus}

Excerpta varia: II.86 [= p. 310].

Excerpta: II.87 [= p. 310].

Protagoras (S. oben Petosiris Excerpta)

Protagorae [Pythagorae] excerpta ex iatromathematicis Mercurii et Petosiridis etc. astrologica (S. oben Petosiris Excerpta [II.82 [= p. 307]): II.87 [= p. 310].

\section{Ptolemaeus}

Excerpta varia: II.87 [= p. 311]; N.64 [= p. 413].

Pythagoras (S. oben Petosiris Excerpta)

Protagorae [Pythagorae] excerpta ex iatromathematicis Mercurii et Petosiridis etc. astrologica (S. oben Petosiris Excerpta [II.82 [= p. 307]): II.88 [= p. 311].

Supputationes prognosticae: II.87 [= p. 311]; N.64 [= p. 413].

\section{Romanus}

\section{Rufus}

De morbis acutis et chronicis: II.88 [= p. 311].

Conspectus de pulsibus: II.90 [= p. 315]; N.65 [= p. 414].

De corporis humani appellationibus: II.89 [= p. 313]; N.65 [= p. 414].

De dissectione partium corporis humani: II.89 [= p. 314].

De medicamentis purgantibus: II.90 [= p. 316]; N.65 [= p. 414].

De ossibus: II.90 [= p. 315].

De satyriasi et gonorrboea: II.88 [= p. 312]; N.64 [= p. 414].

De vesicae renumque affectibus: II.88 [= p. 312]; N.64 [= p. 413].

Excerpta varia: II.91 [= p. 316]; N.65 [= p. 415].

Nomenclatura rerum ad hominem pertinentium. Libri III: II.89 [= p. 314].

Opera varia: N.64 [= p. 413].

Quaestiones medicinales: II.90 [= p. 315].

\section{Salomo}

Praecepta: II.91 [= p. 317].

\section{Severianus}

De hominis nomine: II.91 [= p. 317]; N.65 [= p. 415].

Definitiones: II.91 [= p. 317].

\section{Severus}

De instrumentis infusoriis seu clysteribus ad Timotheum: II.92 [= p. 318].

\section{Socrates}

Excerpta: II.92 [= p. 318]. 


\section{Soranus}

De fasciis: II.92 [= p. 319]; N.65 [= p. 415].

De matrice et muliebri pudendo: II.93 [= p. 320].

De muliebribus affectionibus: II.92 [= p. 318].

De ponderibus et mensuris: II.93 [= p. 320].

De signis fracturarum: II.92 [= p. 319].

Excerpta varia: II.94 [= p. 321].

Hippocratis genus et vita secundum Soranum: II.93 [= p. 319]; N.65 [= p. 415].

\section{Splenius Philosophus}

De generatione hominis: II.94 [= p. 321].

\section{Stephanus Alexandrinus}

De magna sacraque scientia solis itineris ad regem Heraclium: II.96 [= p. 323];

N.66 [= p. 416].

De mundo. Libri IX: II.95 [= p. 322].

De sacra divinaque arte aurifaciendi: II.95 [= p. 322]; N.66 [= p. 416].

Epistula ad Theodorum: II.95 [= p. 323].

In Magni sophistae librum de urinis: II.96 [= p. 324].

Prooemium in Hippocratis de fracturis: II.96 [= p. 324].

Scholia in Hippocratis praenotiones. Sectiones III: II.96 [= p. 323].

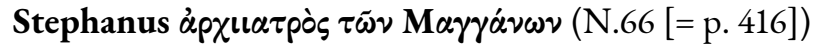

Epistula: II.98 [= p. 327].

Excerpta varia: II.98 [= p. 327].

\section{Stephanus Atheniensis}

De febrium differentiis ex ore Theophili: II.97 [= p. 326]; N.66 [= p. 416].

Dioscoridis et Stephani Atheniensis de pharmacis ordine litterarum: II.97 [= p. 326].

Enarratio Galeni de methodo medendi ad Glauconem librorum: II.97 [= p. 325];

N.66 [= p. 416].

Excerpta varia: II.98 [= p. 327].

In aphorismos Hippocratis commentarius: II.97 [= p. 325]; N.66 [= p. 416].

\section{Stephanus Magnes}

Empirica: II.99 [= p. 327].

Scholia in (Theophili?) tractatum de urinis: II.99 [= p. 328].

\section{Symeon S[anctus] Mamas}

De mutationibus quae corpori et animae accidunt ex aëre, elementis et cibis: II.99

$$
[=\mathrm{p} .328] \text {. }
$$

\section{Syncellus}

\section{Synesius}

Excerpta: II.99 [= p. 328]; N.66 [= p. 417].

Ad Dioscorum, scholia in librum Democriti: II.100 [= p. 329]; N.67 [= p. 418].

De febribus: N.66 [= p. 417].

De insomniis: II.99 [= p. 328]; N.67 [= p. 417]. 
Epistula de silpbio ad fratrem: II.100 [= p. 329]; N.67 [= p. 418].

Taronitus (?)(see N.47 [= p. 389]: Constantinus Taronites)

Excerpta: II.100 [= p. 329]; N.67 [= p. 418].

Theodoretus (N.67 [= p. 418])

Antidota: II.100 [= p. 330].

Theodorus (N.67 [= p. 419])

Excerpta: II.100 [= p. 330].

\section{Theophilus}

Commentarius in Hippocratis Aphorismos: II.104 [= p. 334].

De alvi excrementis: II.102 [= p. 332]; N.68 [= p. 419].

De arte medica, ex Hippocrate et Galeno: II.105 [= p. 335]; N.68 [= p. 419].

De hominis fabrica libri V: II.101 [= p. 330].

De principiis mundi collectanea: II.101 [= p. 331].

De pulsibus: II.101 [= p. 331].

De urinis: II.102 [= p. 333].

Epitome librorum Galeni de febrium differentiis: II.105 [= p. 335].

Excerpta varia: II.106 [= p. 336]; N.68 [= p. 419].

Therapentica Theophili, quam ipse ex variis libris valetudinariis collegit: II.101

[= p. 331].

\section{Theophylactus Simocatta}

Quaestiones naturales: II.106 [= p. 337]; N.68 [= p. 419].

Versus in Galenum: II.106 [= p. 336].

\section{Theopompus}

Theosebius

Excerpta: II.106 [= p. 337].

Excerpta: II.106 [= p. 337].

\section{Timocrates}

Excerpta: II.107 [= p. 337].

\section{Timotheus Gazaeus}

De animalibus quadrupedibus physicisque eorum facultatibus: II.107 [= p. 337].

Vincentius Damodes (N.68 [= p. 419])

Physiologia: II.107 [= p. 338].

\section{Xenocrates}

De alimento ex aquatilibus: II.108 [= p. 338]; N.68 [= p. 420].

De morte (Platonis Axiochus?): II.108 [= p. 339].

\section{Ioannes Zacharias Actuarius}

De animalibus nocentibus: II.110 [= p. 344]; N.69 [= p. 420].

De methodo medendi libri VI: II.109 [= p. 342]; N.69 [= p. 420].

De spiritu animali libri II: II.108 [= p. 339].

De urinis libri VII: II.109 [= p. 340].

Excerpta varia: II.111 [= p. 344]; N.69 [= p. 420]. 
In Galenum de methodo medendi: II.111 [= p. 344].

Opera varia: II.108 [= p. 339].

Tractatus de urinis, qui ex Avicenna translatus dicitur in graecam linguam a Christodulo, in ordinem redactus ab Actuario Zacharia: II.110 [= p. 344].

\section{Zenarius}

Excerpta: II.111 [= p. 345].

Exordia: N.69 [= p. 420].

\section{Zosimus Panopolita}

De instrumentis et fornacibus: II.112 [= p. 346].

De sacra divinaque arte auri et argenti faciendi: II.112 [= p. 346].

Excerpta/Excerpta varia: II.112 [= p. 346]; N.69 [= p. 421].

Gemini commentarii de aqua divina: II.112 [= p. 345].

Liber de virtute et compositione aquarum: II.111 [= p. 345]; N.69 [= p. 421]. 



\section{Index of Manuscripts}

This index lists all the manuscripts mentioned in Diels. The identification of the manuscripts exactly reproduces that of Diels, which usually comprises the following three or four elements: city (German form), library (most of the time in the original language, often in an abbreviated form and in all cases in their form in Diels' time), collection (when appropriate), and shelfmark or reference to an earlier work. In some cases, the name of the library is not explicitly mentioned; it can be identified through the list of catalogues in Diels' bibliography where library names appear. All such elements of identification are reproduced here in boldface for purposes of clarity. For example: Florenz: Laurent. plut. 74, 1.

For each item in Diels, this index provides the reference to its mention(s) in Diels' lists as described above (p. xxxv) with the following abbreviations: "I" for the first part, devoted to Hippocrates and Galen (published in 1905), "II" for the second part, devoted to all the other physicians (published in 1906), and "N" for the supplement (N for Nachtrag = Supplement; published in 1908), followed by the page numbers (in Arabic numerals). A reference appearing as II.3, for example, indicates a manuscript mentioned in part II, page 3. In some cases, the page number is followed by a number between parentheses, e.g., II.106 (2). This indicates that the item in reference appears twice on the same page.

A Table of Concordance lists the page numbers of Diels (in the left column) with their equivalent in the present volume (in the right column).

This index is preceded by a Finding Aid which provides the city and library names in alphabetical order with reference to the present index of manuscripts, and two lists: one with the names as in Diels and the other with their current standard English name. 

Table of Concordance

\begin{tabular}{|c|c|c|c|}
\hline $\mathrm{I} .3=7$ & $\mathrm{I} .57=90$ & $\mathrm{I} .100=144$ & II. $4=202$ \\
\hline $\mathrm{I} .4=8$ & I. $58=93$ & $\mathrm{I} .101=145$ & II. $5=203$ \\
\hline I. $5=9$ & I. $59=93$ & $\mathrm{I} .102=147$ & II. $6=205$ \\
\hline $\mathrm{I} .8=12$ & $\mathrm{I} .60=95$ & $\mathrm{I} .103=148$ & II.7 = 207 \\
\hline $\mathrm{I} .10=13$ & $\mathrm{I} .61=96$ & $\mathrm{I} .104=150$ & II. $8=209$ \\
\hline $\mathrm{I} .11=15$ & $\mathrm{I} .63=98$ & $\mathrm{I} .105=151$ & II. $9=210$ \\
\hline $\mathrm{I} .12=18$ & $\mathrm{I} .64=98$ & $\mathrm{I} .106=152$ & II. $10=212$ \\
\hline $\mathrm{I} .13=21$ & $\mathrm{I} .65=100$ & $\mathrm{I} .107=153$ & II. $11=213$ \\
\hline $\mathrm{I} .14=24$ & $\mathrm{I} .66=100$ & $\mathrm{I} .108=155$ & II. $12=215$ \\
\hline $\mathrm{I} .17=25$ & $\mathrm{I} .67=101$ & $\mathrm{I} .109=156$ & II. $13=216$ \\
\hline $\mathrm{I} .18=25$ & $\mathrm{I} .68=103$ & $\mathrm{I} .110=157$ & II. $14=216$ \\
\hline $\mathrm{I} .19=28$ & I. $69=105$ & I.111 = 159 & II.15 = 217 \\
\hline $\mathrm{I} .20=29$ & $\mathrm{I} .70=105$ & $\mathrm{I} .112=161$ & II. $16=219$ \\
\hline $\mathrm{I} .21=32$ & $\mathrm{I} .71=107$ & $\mathrm{I} .113=163$ & II. $17=219$ \\
\hline $\mathrm{I} .22=34$ & $\mathrm{I} .72=108$ & $\mathrm{I} .114=165$ & II. $18=221$ \\
\hline $\mathrm{I} .23=36$ & I. $73=110$ & I.115 = 167 & II. $19=224$ \\
\hline $\mathrm{I} .24=39$ & $\mathrm{I} .74=112$ & $\mathrm{I} .116=168$ & II. $20=225$ \\
\hline $\mathrm{I} .25=42$ & $\mathrm{I} .75=113$ & $\mathrm{I} .117=169$ & II. $21=226$ \\
\hline I. $26=44$ & I. $76=114$ & $\mathrm{I} .118=170$ & II. $22=227$ \\
\hline I. $27=46$ & I. $77=115$ & $\mathrm{I} .119=171$ & II. $23=229$ \\
\hline $\mathrm{I} .28=49$ & $\mathrm{I} .78=116$ & $\mathrm{I} .120=173$ & II. $24=229$ \\
\hline $\mathrm{I} .29=51$ & $\mathrm{I} .79=118$ & $\mathrm{I} .121=174$ & II. $25=231$ \\
\hline $\mathrm{I} .30=54$ & I. $80=119$ & $\mathrm{I} .122=175$ & II. $26=232$ \\
\hline $\mathrm{I} .31=56$ & $\mathrm{I} .81=121$ & $\mathrm{I} .123=176$ & II. $27=233$ \\
\hline $\mathrm{I} .32=59$ & $\mathrm{I} .82=122$ & $\mathrm{I} .124=178$ & II. $28=234$ \\
\hline $\mathrm{I} .33=61$ & $\mathrm{I} .83=124$ & $\mathrm{I} .125=179$ & II. $29=235$ \\
\hline $\mathrm{I} .34=63$ & $\mathrm{I} .84=125$ & $\mathrm{I} .126=181$ & II. $30=237$ \\
\hline $\mathrm{I} .35=66$ & $\mathrm{I} .85=126$ & $\mathrm{I} .127=182$ & II. $31=239$ \\
\hline $\mathrm{I} .36=68$ & $\mathrm{I} .86=127$ & $\mathrm{I} .128=183$ & II. $32=239$ \\
\hline $\mathrm{I} .37=70$ & $\mathrm{I} .87=128$ & $\mathrm{I} .129=184$ & II. $33=242$ \\
\hline I. $38=71$ & I. $88=129$ & $\mathrm{I} .130=185$ & II. $34=243$ \\
\hline $\mathrm{I} .39=73$ & I. $89=130$ & $\mathrm{I} .131=186$ & II. $35=245$ \\
\hline $\mathrm{I} .40=74$ & I. $90=132$ & $\mathrm{I} .132=187$ & II. $36=246$ \\
\hline $\mathrm{I} .41=76$ & I. $91=133$ & $\mathrm{I} .133=188$ & II. $37=248$ \\
\hline $\mathrm{I} .42=78$ & I. $92=134$ & $\mathrm{I} .134=190$ & II. $38=249$ \\
\hline $\mathrm{I} .43=80$ & $\mathrm{I} .93=135$ & $\mathrm{I} .135=191$ & II. $39=251$ \\
\hline I. $44=82$ & I. $94=136$ & $\mathrm{I} .136=192$ & II. $40=252$ \\
\hline $\mathrm{I} .46=83$ & I. $.95=137$ & I. $148=192$ & II. $41=254$ \\
\hline $\mathrm{I} .47=85$ & I.96 = 138 & $\mathrm{I} .149=193$ & II. $42=256$ \\
\hline $\mathrm{I} .48=86$ & I.97 = 141 & $\mathrm{I} .150=194$ & II. $43=256$ \\
\hline $\mathrm{I} .49=87$ & I. $98=141$ & $\mathrm{I} .151=197$ & II. $44=258$ \\
\hline I. $56=89$ & I.99 $=142$ & II.3 = 201 & II. $45=260$ \\
\hline
\end{tabular}




\begin{tabular}{|c|c|c|c|}
\hline II. $47=261$ & II. $75=294$ & II $.105=335$ & $\mathrm{~N} .42=383$ \\
\hline II. $48=261$ & II. $76=296$ & II.106 = 336 & $\mathrm{~N} .43=384$ \\
\hline II. $49=262$ & II. $77=297$ & II. $107=337$ & N.44 $=385$ \\
\hline II. $50=264$ & II.78 = 299 & II $108=338$ & N.45 $=387$ \\
\hline II. $51=265$ & II.79 = 301 & II. $109=340$ & $\mathrm{~N} .46=388$ \\
\hline II. $52=266$ & II. $80=303$ & II. $110=342$ & $\mathrm{~N} .47=389$ \\
\hline II. $53=267$ & II $.81=305$ & II.111 = 344 & $\mathrm{~N} .48=390$ \\
\hline II. $54=268$ & II. $82=306$ & II.112 = 345 & N.49 $=391$ \\
\hline II. $55=268$ & II. $83=308$ & II.113 = 349 & N.50 $=393$ \\
\hline II. $56=270$ & II. $85=308$ & II. $114=350$ & N.51 $=394$ \\
\hline II. $57=270$ & II. $86=309$ & II. $115=353$ & N.52 $=396$ \\
\hline II. $58=272$ & II. $87=310$ & N.25 = 359 & N.53 $=397$ \\
\hline II. $59=273$ & II. $88=311$ & N.26 $=359$ & N.54 $=399$ \\
\hline II. $60=274$ & II. $89=313$ & N.27 = 361 & N. $55=400$ \\
\hline II.61 = 275 & II. $90=315$ & N.28 $=362$ & N.56 $=401$ \\
\hline II. $62=276$ & II.91 = 316 & N.29 $=363$ & N. $57=403$ \\
\hline II.63 = 277 & II.92 = 318 & N.29 $=367$ & N. $58=404$ \\
\hline II. $64=279$ & II.93 = 319 & N.30 $=367$ & N.59 $=405$ \\
\hline II. $65=281$ & II.94 = 321 & N.31= 368 & N.60 $=407$ \\
\hline II. $66=282$ & II.95 = 322 & N.32 $=370$ & N.61 $=408$ \\
\hline II. $67=283$ & II.96 = 323 & N.33 = 371 & N.62 $=409$ \\
\hline II.68 = 286 & II.97 = 325 & N.34 $=372$ & $\mathrm{~N} .63=411$ \\
\hline II. $69=287$ & II.98 = 326 & N.35 $=373$ & N.64 $=412$ \\
\hline II.70 = 288 & II.99 = 327 & N.36 $=374$ & N.65 $=414$ \\
\hline II.71 = 290 & II. $100=329$ & N.37 = 376 & N.66 $=416$ \\
\hline II.72 = 292 & II.101 = 330 & N.38 $=377$ & $\mathrm{~N} .67=417$ \\
\hline II.73 = 293 & II. $102=332$ & N.39 $=379$ & $\mathrm{~N} .68=419$ \\
\hline II.74 = 293 & II. $104=334$ & $\mathrm{~N} .41=380$ & $\mathrm{~N} .69=420$ \\
\hline
\end{tabular}




\title{
Finding Aid
}

\section{City and Library names}

\author{
1. Names according to Diels
}

[Amsterdam, Universitätsbibliothek]: xcvii Antwerpen, Stadtbibliothek: xcvii Athen: xcvii

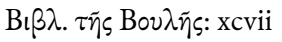

Nationalbibl.: xcvii

Athos: xcvii

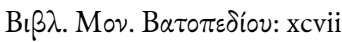

Bı $\beta$. Mov. $\Delta$ เovvбiov: xcviii

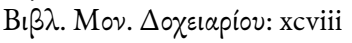

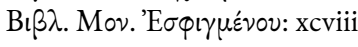

Bı $\beta \lambda$. Mov.' 'I $\beta \dot{n} \rho \omega v$ : xcviii

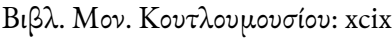

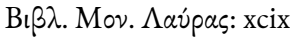

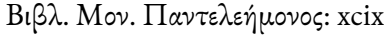

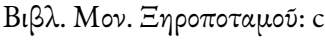

Паvтокра́тороక: $\mathrm{c}$

$\sum \tau \alpha \nu$ poviкnं $\tau \alpha: c$

$\Xi \varepsilon \nu \circ \varphi \tilde{\omega} \nu \tau \leftarrow \circ: \mathrm{c}$

Basel, Bibl. univ.: c

Berlin: $\mathrm{c}$

Pap.: c

Königl. Bibl.: c

$4 \%$ qu.: c

fol.: c

Hamilton.: c

Phillipps.: c

Bern, Stadtbibliothek: ci

Bologna: cii

Bibl. Comunale: cii

Bibl. Universitaria: cii

Breslau, Bibl. urb.: cii

Brüssel, Königl. Bibl.: cii

Budapest: cii

[Bibl. Regia]: ciii

Mus. nat. Hungar.: cii

Cairo: ciii

Ad. Cattavi: ciii
Patriarch. Alexandrin.: ciii

Cambridge: ciii

[Ashmol.]: ciii

Caius Coll.: ciii

Cantabrig. Bibl. Univ.: ciii

[Cath. Metens.]: ciii

Coll. St. Johann.: ciii

[Pembroch.]: civ

[St. Petri]: civ

Trinity Coll.: civ

Carpentras, Bibl. Inguimbertine: civ

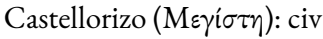

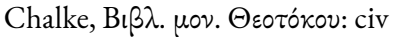

Cheltenham, Phillipps.: civ

[Corbie, Bibl. S. Petri Corbeiens.]: civ

Dresden, Königl. Bibl.: civ

Dublin: cv

[Bibl. Narcissi]: cv

[Coll. Trinit.]: $\mathrm{cv}$

Edschmiadzin: cv

Erlangen, Universitätsbibliothek: cv

Escurial: $\mathrm{cv}$

Florenz: cvi

Bibl. Marucell.: cvi

Bibl. Mediceo Laurentiana: cvi

Conv. soppr.: cvi

Laurent. App.: cviii

Laurent. plut.: cvi

Bibl. Magliabech./bibl. naz.: cix

Bibl. Riccardiana: cix

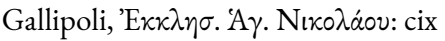

Genf, Genev. Bibl. Urb.: cix

Genua, Bibl. della Missione urbana: cix

Glasgow, Hunterian Museum: cix 
Göttingen, Univ.-Bibl.: cix

Hist. nat.: cix

Philol.: cix

Grottaferrata, Klosterbibliothek: cx

Hamburg, Stadtbibliothek: cx

Heidelberg, Bibl. Univ.: cx

Holkham, Bibl. des Gr. Leicester: cx

Jena, Universitätsbibliothek: cx

Jerusalem: $\mathrm{cx}$

Bibl. d. hl. Grabes: cx

Bibl. d. Hl. Kreuzes: cx

Bibl. Mar-Saba: cxi

Bibl. patriarch.: cxi

Karlsruhe, Großherzogl. Hof. u.

LandesbibliothekL cxi

Königsberg Pr., Kgl. u. Univ. Bibl.: cxi

Dietz-Nachlaß: cxi

Regimont.: cxi

Konstantinopel: cxi

[ap./apud Verdier]: cxi

Bibl. d. Serail: cxi

[Cod. Mich. Cantacuzeni]: cxi

Kopenhagen: cxi

Bibl. Univ.: cxi

e donatione variorum: cxi

$$
\begin{aligned}
& 4^{\mathrm{o}} \text { : cxi } \\
& \text { fol.: cxii }
\end{aligned}
$$

Königl. Bibliothek: cxii

ant. fund. reg.L cxii

Krakau, Bibl. Univ. Jagellon.: cxii

Leipzig: cxii

Bibl. senat.: cxii

Institut f. Geschichte der Medizin: cxii

Universitätsbibliothek: cxii

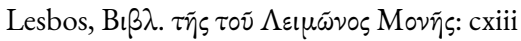

Leyden, Universitätsbibliothek: cxiii
B. P.: cxiii
Gron.: cxiii
Miscell.: cxiii
Ruhnken: cxiii
Scal.: cxiii
Vossian./Voss.: cxiii
Voss.: cxiii
Voss. $4^{\circ}$ : cxiii

Voss. $8^{\circ}$ : cxiv

Voss. fol.: cxiv

Voss. Miscell.: cxiv

Vulc.: cxiv

[Warnerian.]: cxiv

London: cxiv

[Bibl. eccl. Westmonast.]: cxiv

British Museum: cxiv

$$
\begin{aligned}
& \text { Addit.: cxiv } \\
& \text { Arundel.: cxv } \\
& \text { Burneian.: cxv } \\
& \text { Harleian.: cxv } \\
& \text { Regius: } \mathrm{cxv} \\
& \text { Sloan.: cxv } \\
& \text { Stowe: } \mathrm{cxv} \\
& \text { Medical Society: } \mathrm{cxv}
\end{aligned}
$$

Lyon, Bibliothèque de la ville: cxvi

Madrid: cxvi

Bibl. Univ.: cxvi

Nationalbibliothek: cxvi

Privatbibliothek des Königs: cxvi

Mailand: cxvi

Arch. del Capitolo Metropolitano: cxvi

Bibl. Ambrosiana: cxvi

Bibl. Trivulziana: cxix

Messina: cxix

Bibl. S. Salvatoris: cxix

Universität: cxix

Mileae, Bibl. Mileens.: cxix

Mitylene, Bibl. gymnas.: cxix

Modena, Bibl. Estense: cxix

Montpellier, Bibliothèque de l'école: cxx

Moskau, Synodialbibl.: cxx

München: cxxi

Bibl. August.: cxxi

Hof- und Staatsbibliothek: cxxi

graec.: cxxi

lat.: cxxii

Neapel: cxxii

Biblioteca Borbonica: cxxii

Bibl. dei Gerolamini: cxxii

Bibl. di S. Giovanni di Carbonara: cxxii

Bibl. Oratoriana: cxxii

Neu-Ephesus, Bibl. Ephes.: cxxii

[Norfolk]: cxxii 
Orléans, Bibl. communale: cxxiii

Oxford: cxxiii

[Ashmol.]: cxxiii

Bibl. Aedis Christi: cxxiii

Bibl. Bodleiana: cxxiii

[bibl. Brit.]: cxxiii

[cat. mss. Angl.]: cxxiii

Auct.: cxxiii

Baroccian.: cxxiii

Canonician.: cxxiv

Clarkian.: cxxiv

Ger. Langbainii Advers.: cxxiv

Is. Casauboni Advers.: cxxiv

Laudian.: cxxiv

Miscell.: cxxiv

Orvillian: cxxv

Roe: $\mathrm{cxxv}$

[Coll. Merton.]: cxxv

[Coll. Novi]: cxxv

[Eccl. Wigorn.]: cxxv

Padua: cxxv

[Bibl. Canonicor. Lateran.]: cxxv

[Bibl. Cathedr.]: cxxv

Bibl. Joann. Rhodii: cxxvi

Bibl. Marci Mantuae: cxxvi

Bibl. S. Joannis in Viridario: cxxvi

Bibl. Tomasini: cxxvi

Palermo, Biblioteca nazionale: cxxvi

Paris: cxxvi

[Bibl. Regia]: cxxvi

Bibl. de la ville de Paris: cxxvi

Bibl. de Paris: cxxvi

Bibl. Facult. Med.: cxxvi

Bibl. Mazarine: cxxvii

Bibl. Nationale: cxxvii

Coislin.: cxxvii

Imprimés: cxxvii

Parisin.: cxxvii

Suppl.: cxxxvii

Parma, Bibl. Palatina: cxxxix

Patmos, Monast. St. Ioann. Evang.: cxxxix

Petersburg, Kaiserl. öffentl. Bibl.: cxxxix

Pistoja, Biblioteca Fabroniana: cxxxix

Raudnitz: cxxxix

Bibl. Ducis Lobcovic: cxxxix

Ravenna, Biblioteca Classens.: cxxxix
[Reims]: cxl

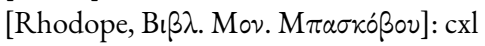

Rodosto: cxl

Rom: cxl

Bibl. Angelica: cxl

Bibl. Barberin.: cxl

Bibl. Corsiniana: cxl

Bibl. St. Petri: cxli

Bibl. Vallicellana: cxli

Bibl. Vaticana: cxli

Ottobon.: cxli

Palat.: cxli

Passioneian.: cxlii

Reg. Suec.: cxlii

Urbin.: cxlii

Vatic.: cxliii

Vatic. lat.: cxlv

Fonds Colonna: cxlv

Rosanbo, Bibl. de M. le marquis de Ros.: cxlv

Salamanca, Bibl. univ.: cxlv

Saloniki, Bibl. gymnas.: cxlv

Sáros-Patak, Bibl. d. reform. Colleg.: cxlvi

Sens, Bibl. communale: cxlvi

Toledo, Bibl. del cab. de la igl. catedr.: cxlvi

Tübingen, Univ.-Bibl.: cxlvi

Turin, Bibl. Nazionale (Universitaria): cxlvi

Upsala, Upsal. bibl. acad.: cxlvi

Urbini et Pisau, Bibl. Ducis: cxlvi

Venedig: cxlvii

Bibl. Andreae Asulani: cxlvii

Bibl. Andreae de Rubeis: cxlvii

Bibl. Josephi de Aromatariis: cxlvii

[Bibl. Mon. St. Michaelis]: cxlvii

Bibl. Monast. S. Michaelis prope

Murianum: cxlvii

Bibl. Nazionale, Marcian.: cxlvii appendix graeca: cxlix graeci: cxlvii

[Bibl. S. Anton.]: cxlix

Verona, Bibl. Capitolare: cxlix

Vicenza, Bibl. Bertoliana: cl

Warschau, Bibl. Zamoyski: cl 
Wien: cl

Bibl. Colleg. S. J. Rossia: cl

Gollob Privatbesitz: cl

Hofbibliothek: $\mathrm{cl}$

[ap. Lamb./Lambec.]: cl

[ap. Nessel]: cl

[Lamb./Lambec.]: cl

histor.: $\mathrm{cl}$

lat.: $\mathrm{cl}$

med.: cli

phil.: clii

philos. et philol.: clii suppl.: clii

theol.: clii

Wolfenbüttel, Bibl. Guelf. August.: clii

[August.]: clii

Guelferb.: cliii

Guelferbyt. Ebert: cliii

Helmstad.: cliii

Weissenb.: cliii

Zaragoza, Bibl. d. Cat. d. Pil.: cliii

Zeitz, Stiftsbibliothek: cliii

Zürich, Stadtbibliothek: cliii

\section{Standard English names}

[Amsterdam, University Library]: xcvii

Antwerp, City Library: xcvii

Athens: xcvii

Parliament Library: xcvii

National Library: xcvii

Athos (Mount -): xcvii

Dionysiou Monastery: xcviii

Dochiariou Monastery: xcviii

Esfigmenou Monastery: xcviii

Iviron Monastery: xcviii

Koutloumousiou Monastery: xcix

Lavra Monastery: xcix

Panteleimonos Monastery: xcix

Pantokrator Monastery: c

Stavronikita Monastery: c

Vatopedi Monastery: xcvii

Xiropotamou Monastery: c

Xenofontos Monastery: c

Basel, University Library: c

Berlin: c

Pap.: c

Royal Library: c

$4 \%$ qu: $c$

fol.: c

Hamilton.: c

Phillipps.: c

Bern, City Library: ci

Bologna: cii

Municipal Library: cii

University Library: cii
Breslau, City Library: cii

Brussels, Royal Library: cii

Budapest: cii

National Museum of Hungary: cii

[Royal Library]: ciii

Cairo: ciii

Ad. Cattavi: ciii

Patriarchate of Alexandria: ciii

Cambridge: ciii

[Ashmol.]: ciii

Caius College: ciii

[Cathedral Metensis]: ciii

[Pembroke College]: civ

St. John's College: ciii

[St. Peter's College]: civ

Trinity College: civ

University Library: ciii

Carpentras, Bibl. Inguimbertine: civ

Castellorizo (Megisti): civ

Chalki, Library of the Theotokou Monastery: civ

Cheltenham, Phillipps.: civ

Constantinople: cxi

[ap./apud Verdier]: cxi

[cod. Mich. Cantacuzeni]: cxi

Serail Library: cxi

Copenhagen: cxi

Royal Library: cxii

ant. fund. reg.: cxii 
University Library: cxi e donatione variorum: cxi

$$
\begin{aligned}
& 4^{\circ} \text { : cxi } \\
& \text { fol.: cxii }
\end{aligned}
$$

[Corbie, Library of St Peter at Corbie]: civ

Cracow, Jagellonian University Library: cxii

Dresden, Royal library: civ

Dublin: cv

[Narcissus Library]: cv

[Trinity College]: cv

Edschmiadzin: cv

Erlangen, University Library: cv

Escorial (San Lorenzo de El -), Monastery

Library: cv

Florence: cvi

Marucelliana Library: cvi

Medicea Laurenziana Library: cvi

Conv. soppr.: cvi

Laurent. App.: cviii

Laurent. plut.: cvi

National Library: cix

Riccardiana Library: cix

Gallipoli, St Nicolas Church: cix

Geneva, City library: cix

Genoa, Urban Mission Library: cix

Glasgow, Hunterian Library: cix

Göttingen, University Library: cix

Hist. nat.: cix

Philol.: cix

Grottaferrata, Monastery Library: cx

Hamburg, City Library: cx

Heidelberg, University Library: cx

Hokham, Library of the Earl of Leicester: cx

Jena, University Library: cx

Jerusalem: $\mathrm{cx}$

Library of the Holy Cross: cx

Library of the Holy Sepulchre: cx

Mar-Sabba Library: cxi

Patriarchal Library: cxi

Karlsruhe, Library of Grand Duchy Court and State: cxi
Königsberg (Prussia), Royal and University

Library: cxi

Dietz-Nachlaß: cxi

Regimont.: cxi

Leipzig: cxii

Institute for the History of Medicine: cxii

Senate Library: cxii

University Library: cxii

Lesvos, Library of the Limonos Monastery: cxiii

Leiden, University Library: cxiii

B. P.: cxiii

Gron.: cxiii

Miscell.: cxiii

Ruhnken: cxiii

Scal.: cxiii

Vossian./Voss..: cxiii

Voss.: cxiii

Voss. $4^{\circ}$ : cxiii

Voss. $8^{\circ}$ : cxiv

Voss. fol.: cxiv

Voss. Miscell.: cxiv

Vulc.: cxiv

[Warnerian.]: cxiv

London: cxiv

British Museum: cxiv

Addit.: cxiv

Arundel.: cxv

Burneian.: cxv

Harleian.: cxv

Regius: cxv

Sloan.: cxv

Stowe: cxv

[Library of Westminster Church]: cxiv

Medical Society: cxv

Lyon, City Library: cxvi

Madrid: cxvi

National Library: cxvi

Royal Library: cxvi

University Library: cxvi

Messina: cxix

S. Salvatore Library: cxix

University Library: cxix

Milan: cxvi

Ambrosiana Library: cxvi

Archives of the Metropolitan Chapter: cxvi

Trivulziana Library: cxix 
Milies, Milies Library: cxix

Modena, Estense Library: cxix

Montpellier, Medical School Library: cxx

Moscow, Synodial Library: cxx

Munich: cxxi

Augustean Library: cxxi

Court and State Library: cxxi

graec.: cxxi

lat.: cxxii

Mytiline, Gymnasium Library: cxix

Naples: cxxii

Girolamini Library: cxxii

Oratory Library: cxxii

Royal Borbonica Library: cxxii

St. John Library at Carbonara: cxxii

Nea-Ephesos, Ephesian Library: cxxii

[Norfolk]: cxxii

Orléans, Municipal Library: cxxiii

Oxford: cxxiii

Aedes Christi Library: cxxiii

[Ashmol.]: cxxiii

Bodleian Library: cxxiii

[bibl. Brit.]: cxxiii

[cat. mss.Angl.]: cxxiii

Auct.: cxxiii

Baroccian.: cxxiii

Canonician.: cxxiv

Clarkian.: cxxiv

Ger. Langbainii Advers.: cxxiv

Is. Casauboni Advers.: cxxiv

Laudian.: cxxiv

Miscell.: cxxiv

Orvillian: cxxv

Roe: $\mathrm{cxxv}$

[Merton College]: cxxv

[New College] : cxxv

[Worcester Church] : cxxv

Padua: cxxv

[Cathedral Library]: cxxv

Johann Rhodius' Library: cxxvi

[Library of Canons Regular of the

Lateran]: cxxv

Marco Mantua's Library: cxxvi

St. John in Viridario Library: cxxvi

Tomasini's Library: cxxvi
Palermo, National library: cxxvi

Paris: cxxvi

Library of the City of Paris: cxxvi

Mazarine Library: cxxvii

Medical School Library: cxxvi

National Library: cxxvii

Coislin.: cxxvii

Imprimés: cxxvii

Parisin.: cxxvii

Suppl.: cxxxvii

Paris Library: cxxvi

[Royal Library]: cxxvi

Parma, Palatine Library: cxxxix

Patmos, Library of the Monastery of St. John

the Evangelist: cxxxix

Pistoia, Fabroniana Library: cxxxix

Raudnitz: cxxxix

Duke Lobkowicz's Library: cxxix

Ravenna, Classense Library: cxxxix

[Reims]: cxl

[Rhodope, Library of Baskobou Monastery]: cxl

Rodosto: cxl

Rome: cxl

Angelica Library: cxl

Barberiniana Library: cxl

Colonna collection: cxlv

Corsiniana Library: cxl

St. Peter's Library: cxli

Vallicelliana Library: cxli

Vatican Library: cxli

Ottobon.: cxli

Palat.: cxli

Passoneian.: cxlii

Reg. Suec.: cxlii

Urbin.: cxlii

Vatic.: cxliii

Vatic. lat.: cxlv

Rosanbo, Marquis of Rosanbo's Library: cxlv

Saint-Petersburg, Public Imperial Library: cxxxix

Salamanca, University Library: cxlv

Sáros-Patak, Library of the Reformed College: cxlvi

Sens, Municipal Library: cxlvi

Thessalonica, Gymnasium Library: cxlv 
Toledo, Library of the Chapter of the Cathedral Church: cxlvi

Tübingen, University Libray: cxlvi

Turin, National Library (University Library): cxlvi

Uppsala, Library of the Academy: cxlvi

Urbino and Pesaro, Duke's Library: cxlvi

Venice: cxlvii

Andrea Asulanus' Library: cxlvii

Andrea de Rubeis' Library: cxlvii

Giuseppe degli Aromatari's Library: cxlvii

[Library of St Michael's Monastery]: cxlvii

Library of St Michael's Monastery in Murano: cxlvii

National library: cxlvii appendix graeca: cxlix graeci: cxlvii

[San Antonio of Castello Library]: cxlix

Verona, Chapter Library: cxlix

Vicenza, Bertoliana Library: cl

Vienna: cl

Court Library: cl

[ap. Lamb./Lambec.]: cl [ap. Nessel]: cl

[Lamb./Lambec.]: cl

histor.: $\mathrm{cl}$

lat.: $\mathrm{cl}$

med.: cli

phil.: clii

philos. et philol.: clii

suppl.: clii

theol.: clii

Private Collection Eduard Gollob: cl

Rossiana Library of Jesuit Company

College: $\mathrm{cl}$

Warsaw, Zamoyski Library: cl

Wolfenbüttel, August Guelph's Library: clii

[August.]: clii

Guelferb.: cliii

Guelferbyt. Ebert: cliii

Helmstad.: cliii

Weissenb.: cliii

Zaragoza, Library of the Cathedral of Our Lady of the Pilar: cliii

Zeitz, Abbey Library: cliii

Zürich, City Library: cliii 



\section{Index of Manuscripts}

[Amsterdam, Universitäts-Bibliothek]

[?]: I.109.

Antwerpen, Stadtbibliothek

426: I.12.

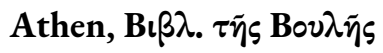

39: N.26 (7).

43: N.58.

68: N.25, 28 (2), 46, 49, 62, 63.

Athen, Nationalbibl.

375: II.66.

433 (132): II.39.

447 (146): II.66.

1180: II.44.

1236: II.27.

1444: II.27.

1477: I.3, 11 .

1478: N.60.

1479: I.123; II.34, 79.

1480: II.29.

1481: II. 55, 68.

1486: I.12.

1488: I.10.

1491: I.78 (2), 79 (2).

1493: I.99, 121.

1494: I.91; II.102.

1498: I.12.

1500: I.12.

1502: I.100.

1504: I.44.

1506: II.64.

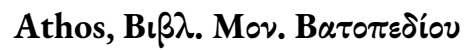

A 29: II.5; N.43. 


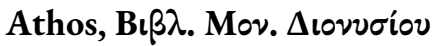

3701.167: I.40, 124.

3748.214: II.39, 40 .

3758.224: I.47.

3766.232: II.35.

3778.244: II.35.

3799.265: II.66.

3808.274: II.39.

3881.347: II.58.

3897.363: II.35.

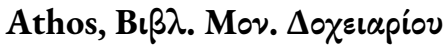

2917.243: I.47.

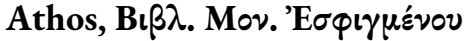

41: I.12, 41, 104; II.5.

2323.310: I.12.

2330.317: I.21.

\section{Athos, Bı $\beta \lambda$. Mov. 'I $\beta \eta ́ p \omega \nu$}

151: II.109.

4212.92: I.40.

4271.151: I.40, 48, 93, 99-100, 117, 121, 132; II.14, 41, 58, 69, 70, 78.

4272.152: II.35.

4284.164: I.61, 91.

4294.174: I.42, 123.

4300.180: I.100.

4301.181: II.3.

4302.181: II.27, 39, 73.

4302.182: I.17, 47; II.91.

4304.184: I.102.

4309.189: I.89, 90, 94, 102.

4310.190: I.56, 148; II.35.

4325.205: II.35.

4330.310: I.41.

4336.216: II.29, 39.

4337.217: II.32, 34.

4338.218: I.39.

4339.219: I.99, 132.

4340.220: I, 44; II.7.

4436.316: II.71, 72, 77. 
4450.330: II.35.

4463.343: I.42, 44 .

4468.348: II.35.

4497.377: II.35.

4501.381: I. 40 .

4508.388: I.132.

4655.535: I.42, 123; II.64.

4671.551: I.12.

4720.600: II.35.

4788.668: II.35.

4789.669: I.136.

4799.679: II.35.

4815.695: II.35.

4871.751: II.36.

4925.805: II.35.

5034.914: I.40, 124.

5437.1317: I.136.

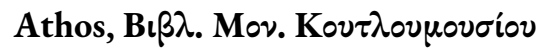

3260.2: II.35.

3321.248: I.111; II.34, 73.

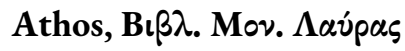

-: II.107.

-: II.29, 31, 32, 33, 38, 39 (2).

Ausser $\Omega 70$ und 72 noch 4 weitere Hdss. bei Costomiris Rev. Des ét. gr.

III p. 166 und 127: II.5.

$\Omega$ 69: II.110.

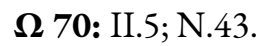

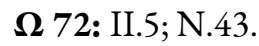

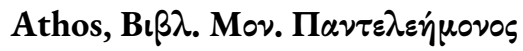

5578.72: I.148.

5752.245: II.35.

5768.261: II.64.

5769.262: I.42, 123; II.64, 79.

5796.289: I.12.

6342.835: I.12.

6351.844: II.91.

6972.865: II.64. 


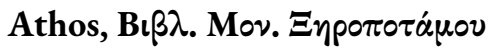

2342.99: I.125.

2432.99: I.39, 46, 124; II.34.

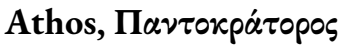

1268.234: I.148.

Athos, $\Sigma \tau \alpha$ upovเหท́ $\tau \alpha$

951.86: II.35.

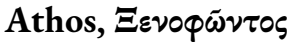

739.37: II.39.

Basel, Bibl. univ.

F VI 46: II.77.

\section{Berlin}

Pap. 6934: I.37.

Pap. 7094: I.37.

\section{Berlin, Königl. Bibl.}

$4^{\circ}$

2 (301): II.61.

5 (304): II.36, 63.

21 (319): I.96.

qu.

5 (304): I.39, 124.

46 (348): II.64.

fol.

7 (247): II.109

35 (271): II.70.

37: N.43.

37 (273): II.5, 8.

38: N.43.

38 (274): II.5.

39 (275): II.7, 10, 13, 15, 16, 17 (2), 18 (2), 20, 22, 34, 37, 73, 79-80, 91, 109.

Hamilton.

270 (401): I.74.

Phillipps.

1432: II.39.

1462: I.40, 124. 
1487: I.47.

1524 (ol. Meerm. 214): I.86; II.102, 104.

1525 (ol. Meerm. 215): I.109; II.76; N.61.

1526 (ol. Meerm. 216): I.100, 111.

1527 (ol. Meerm. 217): I.47, 68; II.40, 85, 101.

1528: II.109.

1529 (ol. Meerm. 220): I.32, 98; II.98.

1530: II.29, 31, 32.

1531 (Meerm. 223): II.17 (2), 18 (2), 64, 102, 109.

1532 (ol. Meerman. 225): I.12; II.17 (2), 18 (2), 70, 83, 92, 97, 102.

1533: II.15 (3).

1533 (ol. Meerm. 226): II.70, 92.

1534 (Meerm. 229): II.5.

1535 (ol. Meerm. 229): II.11.

1536 (ol. Meerm. 231): II.88, 89 (2), 90.

1537 (ol. Meerman. 233): I.12.

1538 (ol. Meerm. 233): I.56; II.24.

1539: II.29.

1540: II.24, 95, 98.

1540 (ol. Meerm. 236): II.100, 111, 112 (2).

1547: II.93.

1557: II.66.

1558: II.9.

1562: N.47.

1566 (ol. Meerm. 269): I.41, 75, 97, 113, 124; II.102.

1567 (ol. Meerm. 270): I.67.

1568 (ol. Meerm. 271): II.49, 72.

1570: I.117; II.68, 78.

1571: I.112; II.20.

1571 (ol. Meerm. 279): II.79, 100.

1576 (Meerm. 286): N.58.

1577 (Meerm. 287): N.36, 51, 52.

1581 (ol. Meerm. 297): II.71.

1582: II.108, 109 (2).

1583: I.40; II.7, 78; N.46, 56, 67.

1583 (ol. Meerm. 300): II.69.

1991 (ol. Meerm. 218): I.78 (2), 79 (2).

Bern, Stadtbibliothek

459: II.89 (2).

579: I.26, 37. 
691: I.72.

Bologna, Bibl. Comunale

A I 13: II.66-67.

\section{Bologna, Bibl. Universitaria}

457 vol. XXIV no. 1: II.24.

1808: I.115, 116, 117, 120, 122, 126, 131, 132; II.5, 20, 34, 38, 41, 58, 73, 76, 83, 91 , 106.

2294: II.9.

2678 (ol. 2280: I.96.

2911: II. 21 .

3232: I.12.

3563: I.37.

3632: I.4, 5, 17, 18, 20, 28, 31, 39, 40, 80, 111, 113, 114, 115, 117, 130, 132; II.5, 7, $13,15,16,20,25,29,34,41,45,(2), 56,59,60,73,75,80,86,87,91,100$, $102,106(2), 110$.

3633: I.40; II.28.

3635: II.9, 22.

3636: I.12, 61, 64, 94, 127, 129, 136; II.27, 77, 80, 92, 102.

3637: I.70.

Breslau, Bibl. urb., Rhedigeran.

?: N.50.

Breslau, Vratisl. Rhedig. bibl. urb.

34: II.67.

\section{Brüssel, Königl. Bibl.}

1871-77: I.37.

5362-64: II.63.

11337-41: II.108, 109-110.

11345-48: I.109; II.37.

11351-52: II.67.

11354: II.39.

Budapest, Mus. nat. Hungar.

9 fol.: II.70, 71, 72, 73. 
[Budapest, Bibl. Regia, Cod. Matthiae Corvini]: I.102.

Cairo, Ad. Cattavi -: N.52.

Cairo, Patriarch. Alexandrin. 46: N.41, 68.

[Cambridge, Ashmol., 7751]: I.5.

Cambridge, Caius Coll. 47: I.68, 74, 91, 122 .

50: I.4, 11, 12, 17, 18, 19, 20, 21, 22, 23 (2), $24(2), 25,26$ (2), 27, 28, 29 (2), 33, 34 (2), 35 (2), 48 (2); II.93.

76: I.11, 29, 80; II.109.

77: II.11.

355: I.5, 12, 73, 79 (2), 111.

360: I.63, 65, 91 .

[946]: I.68, 120.

[948]: I.111.

[949]: I.80.

[954]: I.5.

[959]: I.21.

[962]: I.12.

[1134]: I.5.

[6605]: I.120.

Cambridge, Cantabrig. Bibl. Univ.

2049 (Kk V 7): II.37.

[2329]: I.5.

F. F. 3. 30: I.96, 118; II.9, 27.

Gg I 2: II.36.

L 1 IV 12: I.21, 25, 26, 37; II.39.

L. L. 5, 4: I.11.

[Cambridge, Cath. Metens.]

[226]: I.5.

Cambridge, Coll. St. Johann.

A6: II.70. 
[Cambridge, Pembroch.] [2055]: I.5, 12.

[Cambridge, St. Petri]

[1866]: I.5, 12.

Cambridge, Trinity Coll.

1386: II.109.

Carpentras, Bibl. Inguimbertine

nr. 1774: I.115; II.73.

nr. 1774 (P., V/P. V.): II.3, 24, 36, 38, 61.

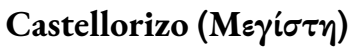

nr. -: I.123.

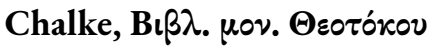

82: I.41.

Cheltenham, Phillipps.

3084: II.30.

3892 (ol. Meerm. 275): II.63.

4614: I.64, 65, 71 (2), 76, 83, 84, 93.

6665-6765 I.96.

6763: II.70 (2), 96.

6774 (ol. Meerm. 298): I.96.

21975: II.30, 31, 32, 34, 39 (2).

23007: II.44.

24, 386: I.89.

[Corbie, Bibl. S. Petri Corbeiens.]

[Montf. p. 1407]: I.148.

Dresden, Königl. Bibl.

Da 1: I.61, 100.

Da 5: II.64, 73, 78, 108, 109, 110, 111; N.69.

Da 57: II.67.

Da 58: II.67.

Da 67: II.108.

Aldina: II.114. 
[Dublin, Bibl. Narcissi]

[1218]: I.148.

[1709]: I.42.

[cod. Brit. 502]: I.10, 19.

[Dublin. Coll. Trinit.]

[502]: I.5, 12.

\section{Edschmiadzin}

nr. ?: II.30.

Erlangen, Universitätsbibliothek

Bibl. Univ. 89: II.44, 45; N.53 (4).

Erlangens. 90: I.12; II.7, 104.

\section{Escurial, Scorial}

- (Puschm. I p. 90): II.12.

III. R. 3: I,73, 132; II.13, 30, 32.

C. II. 11: I.96.

C. III. 17: I.96.

R. I. 12: II.5, 77, 78, 79.

I. I. 12: I.81; II.50, 89.

I. I. 17: II.30, 31, 32, 38, 39 (3).

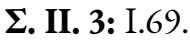

I. II. 5: I.17, $79(2), 102$.

I. II. 10: I.97.

I. II. 11: I.100, 103.

¿. III. 17: I.100, 111, 117, 127; II.5, 7, 67, 105.

T. II. 12: I.44; II.30.

T. II 14: II.47.

T. II. 14: I.26; II.5, 53, 75, 97-98, 109.

T. III. 7: I.67 (3), 69, 108.

Y. I. 9: II.9.

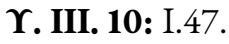

エ. III. 14: I.114, 127; II.28, 42, 53, 59, 77, 78, 102 (2), 110.

ฯ III 17: I.5.

Ү. III. 17: I.104, 107; II.24.

y. I. 8: I.12, 17, 18; II.17 (2), 18 (2), 76, 104.

y. I. 9: II.37, 71 .

y. I. 15: II.62, 63.

y. III. 16: I.32. 
y. III. 18: II.95.

Ф. I. 2: II.4, 8, 11, 71, 77, 89 (2).

Ф. I. 2 (?): II.12.

Ф. I. 6: II.5.

Ф. I. 10: I.151; II.27, 34, 62.

Ф. I. 11: II.24, 95.

Ф. II. 15: II.5.

Ф. III. 7: I.5, 12, 104, 107; II.33, 57.

Ф III 11: I.73.

Ф. III. 11: I.43, 78 (2), 79 (2), 82, 93, 96, 100, 113; II.40, 102.

Ф. III. 12: I.5, 12, 22, 44, 127; II.77, 78, 108, 109, 110 (2), 111.

X. I. 11: II.9, 67.

X. II. 2: II.36.

X. II. 9: II.67.

Y. I. 13: II.24, 95.

Y. III. 7: II.39.

Y. III. 17: I.12.

Y. IV. 14: II.5.

ת. I. 8: II.3, 5, 13, 20, 34, 77.

Florenz, Bibl. Marucell.

A 109: I.18.

Florenz, Bibl. Mediceo Laurentiana

Conv. soppr.

59: II.30.

153: I.37.

163: I.120.

627 (Abb. 2728): II.94.

\section{Laurent.}

-: II.73.

[ap. Bandini III 122]: I.117.

plut. 4, 10: II.94.

plut. 7, 2: II.67.

plut. 7, 35: II.67.

[plut. 25, ?]: I.20, 33 .

plut. 28, 13: N.36, 53.

plut. 28, 14: I.112; II.82; N.36, 52.

plut. 28, 33: N.53.

plut. 28, 34: I.112, 135; II.82; N.36, 53, 62, 69.

plut. 28, 44: II.3. 
plut. 56, 1: II.3.

plut. 56, 15: I.72, 134 .

plut. 56, 21: II.67.

plut. 57 (?), 19: I.115.

plut. 57, 50: II.20.

plut. 58, 2: I.110.

plut. 58, 24: II.94.

plut. 59, 14: I.12, 29, 70, 82, 89, 101 (2); II.51, 83, 96; N.34, 36, 37, 63.

plut. 59, 17: II.20.

plut. 71, 1: I.33.

[plut. 73, 1]: I.48.

[plut. 73, 7]: I.12.

[plut. 73, 8]: I.12.

plut. 73, 9: I.111.

[plut. 73, 22]: I.12.

[plut. 74, (?)]: I.81.

plut. 74, 1: I.4, 5, 8, 10, 11 (3), 12 (2), 17, 18, 19 (2), 20 (3), 21, 22, 23 (2), 24

(3), 25 (2), 26 (2), 27 (3), 28 (2), 29 (3), 30 (2), 31 (2), 33, 34 (3), 35 (2), 38,$109 ;$ II.37, 93.

plut. 74, 2: I.121; II.26, 50, 75, 77; N.47, 61.

plut. 74, 3: I.17, $59(2), 60(2), 68,69,70,71,72,73,74(2), 83,95,106(2)$, 109-110, 110 (3), 111, 121; N.36.

plut. 74, 4: I.68.

plut. 74, 5: I.60, 63, 64, 65, 70, 72, 73, 81, 83 (2), 86, 99 (2), 100.

plut. 74, 6: I.91.

plut. 74, 7: I.10, 11 (3), 67, 91, 106, 107, 126; II.15 (2), 16, 20, 42, 71, 73, 76, $77,89,90,91,92(2) ;$ N.61, 65.

plut. 74, 8: I.104, 107.

plut. 74, 9: I.68.

plut. 74, 10: I.39, 66, 124-125, 130, 132; II.11, 32, 102; N.30, 49.

plut. 74, 11: I.5, 12, 82; II.75, 76, 91, 102, 104; N.61.

plut. 74, 12: I.63, 68, 74, 79, 82, 90, 91, 101; N.34.

[plut. 74, 12]: I.56.

plut. 74, 13: I.12, 17; II.50, 93, 109.

plut. 74, 14: I.100, 111.

plut. 74, 15: II.71.

plut. 74, 16: I.78 (2), $79(2), 85$.

plut. 74, 17: I.96; II.30, 71 (2), 77.

plut. 74, 18: I.68, 87 (2), 88 (2).

plut. 74, 19: I.61.

plut. 74, 20: II.32; N.49. 
plut. 74, 21: I.24; II.77.

plut. 74, 22: I.74, 94-95, 95, 112.

plut. 74, 23: II.8, 30, 32 (2), 77, 78, 79; N.49 (2).

plut. 74, 24: II.77.

plut. 74, 25: I.77, 98, 104; N.32.

plut. 74, 26: II.77.

plut. 74, 27: II.77.

plut. 74, 28: I.87 (2), 88 (2).

plut. 74, 29: II.77.

plut. 74, 30: I.85.

plut. 74, 31: I.35, 82, 87, 107, 127; II.77, 101, 102.

plut. $75,2:$ II.5.

plut. 75, 3: I.5; II.61, 65.

plut. 75, 4: II.78.

plut. 75, 5: I.102, 107; II.5-6; N.35, 43.

plut. 75, 6: II.29.

plut. 75, 7: I.95, 119; II.6, 15, 90 (2), 92; N.43.

plut. 75, 8: I.97; II.7, 27, 33, 34, 110.

plut. 75, 9: I.93; II.78-79, 108, 109.

plut. 75, 10: I.44, 114, 117; II.6, 52; N.43 (2).

[plut. 75, 10]: I.5.

plut. 75, 11: II.79, 108, 109, 110.

plut. 75,12 : II.6.

plut. 75, 13: II.6, 9.

plut. 75, 14: I.63, 80; II.10.

plut. 75, 15: II.17 (2), 18 (2).

plut. 75, 16: I.93; II 22, 79, 108, 109, 110.

plut. 75, 17: I.97, 132.

plut. 75, 18: II.6; N.43.

plut. 75, 19: I.56, 121, 127; II.24, 63, 101.

plut. 75, 20: II.6; N.43.

plut. 75, 21: II.6, 76; N.61.

plut. 75, 22: II.76; N.61.

plut. 86, 6: II.67.

plut. 86, 14: II.82 (2).

plut. 86, 16: II.100, 115.

plut. 86, 20: II.98, 105; N.66.

plut. 87, 16: II.20.

\section{Laurent. App.}

2: I.22, 42 (2), 43 (2), 122, 123, 125, 127; II.3, 12, 13, 14, 22, 27, 33, 35, 38, $42,47,64,79,80,98,100$. 
Florenz, Magliabech.

1: I.44, 127.

44: II.79.

Florenz, bibl. naz. [Magliabecchiana]

II.III.304: II.39.

II.III.428: I.44, 56.

Florenz, Bibl. Riccardiana

10 (K I 12): II.11.

17 (K I 24): I.107.

31 (K II 4): II.108.

41 (K II 2): I.12, 44; N.37.

44 (K II 5): I.5, 12, 77, 104, 107, 112.

71 (K II 16): II.79.

91 (K II 7): II.32; N.49.

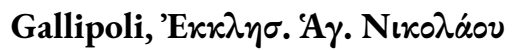

38: I.5, 41, 127, 131; II.7, 59-60, 96, 102.

Genf, Genev. Bibl. Urb.

42: I.96.

Genua, Bibl. della Missione urbana

17: II.39.

Glasgow, Hunterian Museum

V.5.11: II.108.

Göttingen, Univ.-Bibl.

Hist. nat.

3: I.3.

4: I.3.

5: II.104.

6: II.34.

90: II.30.

Philol.

2: I. 49 .

21: I.49. 
Grottaferrata, Klosterbibliothek

2 Г VI (series IX cod. 46): I.67 (3), 109.

Hamburg

nr. - (Vgl. Fabricius bibl. gr. IX. p. 454-474): II.108.

Hamburg, Stadtbibliothek

Hamgurgens. 200: N.68.

Uffenbach. 105: I.71.

\section{Heidelberg, Bibl. Univ.}

132: I.38.

155: I.41.

375: II.71.

398: I.38.

Holkham, Bibl. des Gr. Leicester

282: I.4, 5, 8, 10 (2), 11 (3), 12-13, 19, 21, 22, 23, 27 (2), 28 (2), 29 (2), 30, 31 (2), $32(3), 33,34,35,46 ;$ N.25 (3), 26 (3).

283: II.6; N.43.

289: II.36.

293: I.13; II.99.

Jena, Universitätsbibliothek

Jenens: N.50.

Aldina c. nott. Cornarii: I.58.

Jerusalem, Bibl. d. hl. Grabes

38: II.88.

179: I.126.

189: I.32.

199: I.125.

273: II.67.

304: II.63.

357: II.63.

363: I.125.

405: II.67.

Jerusalem, Bibl. d. hl. Kreuzes

68: I.125.

85: I.148. 
Jerusalem, Bibl. Mar-Saba

366: II.39.

432: I.42, 123; II.64.

481: I.13, 105 .

498: I.5, 13, 20, 33.

Jerusalem, Bibl. patriarch.

15: II.67.

102: I.3, 13, 104-105.

108: II.9.

148: I.42, 115, 136; II.41, 97.

203: II.63.

273: II.7, 77, 91.

339: II.79.

463: I.56.

470: II.63.

511: II.63.

Karlsruhe, Großherzogl. Hof. u. Landesbibliothek 449: I.8, 13, 28, 29.

451: II.67.

Königsberg Pr., Kgl. u. Univ. Bibl.

16 b 12: N.37.

Dietz-Nachlaß

I 1-3: I.3.

I 4: I.3.

Regimont. S. 35: I.151.

Konstantinopel

[ap. Verdier, II 57]: I.148.

[apud Verdier]: I.124.

Cod. Mich. Cantacuzeni: I.116, 123, 132.

[Cod. Mich. Cantacuzeni]: I.116.

Konstantinopel, Bibl. d. Serail

11: I.3, 58 .

Kopenhagen, Bibl. Univ.

e donatione variorum $4^{\circ}$

42: I.61. 
277: II.32.

e donatione variorum fol.

14: I.89.

29: II.97.

Kopenhagen, Königl. Bibliothek

Hauniens. ant. fund. reg.

224: I. $4,5,8,10,11$ (3), 12, 13, 17, 18, 19 (2), 20 (3), 21, 22 (2), 23 (2), 24 (3), 25 (2), 26 (2), 27 (3), 28 (2), 29 (3), 30 (2), 31 (2), 33 (2), 34 (3), 35 (2), $38,39,110 ;$ II.93.

225: I.66, 98, $99(2), 148$.

1648: I.111.

Krakau, Bibl. Univ. Jagellon.

2526 FF VI 5: II.55, 63, 82, 102.

Leipzig, Bibl. senat.

391: II.6; N.43.

Leipzig, Institut f. Geschichte der Medizin

Basileensis des Galen: N.29.

Leipzig, Universitätsbibliothek

50: $\mathrm{I} .75,85$.

51: I.79, 96.

52: I.66, 100, 111.

53: I.93.

54: I.109.

55: I.61.

56: I.103.

57: I.58, 148.

58: I.58.

59: II.15, 71.

60: II.110.

61: I.3.

62: II.77.

63 (ol. 1109?): II.17, 17-18, 18 (2).

64: II.15.

65: II.6.

66: II.24, 95. 


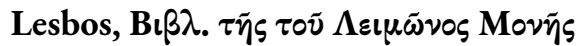

175: I.56, 148; II.36, 40, 64.

268: II.20.

\section{Leyden, Universitäts-Bibliothek}

(Catal. p. 395) II.88, 90.

B. $\mathbf{P}$.

2 A: I.93; II.97.

6: II.6.

16: I.96.

62 A: II.66.

670: I.3.

\section{Gron.}

12: II.39.

Miscell.

11: I.91-92. 93.

\section{Ruhnken}

11: II.63.

Scalig.

18: I.63, 64, 65, 78, 79 (2).

18 (?): I.78.

71: I.5, 13, 56.

Vossian./Voss.

-: II.44.

[-]: I.120.

[?]: I.79.

[ ?]: I.92.

[Cat. Bibl. Lugd. Bat. P. 398 n. 50]: I.123.

9: II.71.

[16]: I.61.

29: II.4.

[31]: I.127.

[58]: I.99.

[2168]: I.87.

[2324]: I.121.

Voss. $4^{\circ}$

9: II.88, 90 .

17: I.61.

38: I.38.

45: I.77, 78, 89, 95, 109, 112 (2).

50: I.43. 
54: II.50.

Voss. $8^{\circ}$

7: I.49.

18: II.7, 92, 93.

20: II.108.

Voss. fol.

10: I.4, 5, 8, 10, 11 (2), 12, 17, 18, 19 (2), 20 (2), 21, 22 (2), 23, 24 (3), 25 (2),

26, 27, 29 (2), 30, 31 (2), 33, 34 (3), 35, 44, 46, 48 (2), 49; II.93.

11: 1.79 .

22: II.77.

25: II.26, 42.

27: I.66.

31: II.101.

32: II.108, 109, 110 .

53: I.85, 93.

58: II.6, 30 .

59: II.33 (2), 34, 101.

65: N.66.

Voss. Miscell.

1 pars 13: I.110.

22: II.108.

Vulc.

43: I.87 (2), 88 (2).

56: I.40.

57: I.66.

108 pars 15: I.49.

[Warnerian.]

[53]: I.127.

[London, Bibl. eccl. Westmonast.]

[1100]: I.89.

\section{London, British Museum}

Addit./Addit. (Brit. Mus.)

5108: II. 29.

6898: I.13, 92.

8231: II.34.

10, 058: I.114, 124, 125, 132-133; II.7, 14, 41, 58, 79, 80, 93.

11, 888: I.68, 111.

14620: N.50.

17148: N.50. 
17, 900: I.127-128, 134; II. 10, 33, 54, 102 (2).

28, 830: I.42, 123.

34,060: I.41.

Arundel./Arundelian.

-: I.93.

537: II.108, 109, 110 .

538: I.13, 17, 18, 20-21, 28, 29.

Burneian.

75: II.61.

94: I.119; II.59, 71, 89 (2).

523: I.58.

\section{Harleian.}

5611: I.105.

5625: I.87 (2), $88(2)$.

5626: I.13, 41, 42, 75, 118, 123; II.3, 6, 15, 16-17, 56, 58, 91.

5635: I. 38.

5651: I.85.

5652: I.68.

5679: II.30, 32 .

5685: II.67.

6295: I.5, 13, 41, 46; II.9, 40, 65.

6301: I.38.

6305: I.89, 90, 117; II.7, 33, 79 (2), 106.

6326: II.17, 18 (3).

\section{Regius}

12 F III: I.111.

16 C XI: I.90; II.19.

16. C. XVI: I.5.

$[1734,4]:$ I.5.

Sloan.

804: II.32.

2434: II.6.

Stowe

1073: I.5, 13, 17, 18, 77.

\section{London, Medical Society}

-: I.150.

AA a 1 = Xa 32: I.40, 41, 47.

A Ac 2 = Wf 15: II.77.

HHi 17 = We 30: I.5, 13, 48, 100, 115, 125, 128, 131, 133; II.7, 79, 80, 98, 101, $102,109$. 
HH i 21. 22 = We 28. 29: I.41, 131; II.7, 102, 108-109, 109, 110.

H Hi 23 = We 32a: II.17, 18 (3), 89 (2).

NNa 11 = Wf 8: II.110.

We 29: II.48.

Wf 6: II.71.

Wf 7: II.6.

Wf 16: II.109.

Lyon, Bibliothèque de la ville

52: II.67.

Madrid

Matrit. 166: II.53.

Madrid, Bibl. Univ.

30: II.36.

Madrid, Nationalbibliothek

4557 (N 119): I.38.

4581 (olim N 45): II.77.

4616 (olim N 48): II.82.

4616 (olim N 84): II.9, 22, 34, 77, 80, 87.

4631 (N 110): I.126; II.7, 34, 36, 41 (2), 43, 77, 79, 80, 87.

4634 (olim N 112): I.5, 13.

4636: II.50.

4636 (ol. N 115): II,89.

4759 (olim N 138): II.28, 67.

4783 (olim O 67): I.112.

4861: II.39.

9715: II.110.

N 116 (olim Toletanus 97.15): II.85.

Madrid, Privatbibliothek des Königs

14 (olim 13): II.39-40.

44 (olim 23): I.122; II.30, 32 (2).

Mailand, Arch. del Capitolo Metropolitano

2: I.117; II.30, 32 (2), 33, 34, 68, 79, 100.

Mailand, Bibl. Ambrosiana

(bei Berthelot Ruelle, p. 56): II.100. 
[ap. Montf. p. 497]: I.58.

( Vgl. Montfaucon I p. 504): II.89.

A 45 Sup.: N.27 (2), 31, 38, 64.

A 80 Sup.: N.41.

A 92 Sup.: N.46.

A 95 Sup.: I.40, 41, 126; II.30, 32, 33, 102, 104; N.41.

A 110 Sup.: I.41.

A 156 Sup.: I.5, 13, 20, 77, 105, 107, 112.

A 157 Sup.: II.7; N.43.

A 162 Sup.: N.44.

A 175 Sup.: N.43.

B 39 Sup.: II.67.

B 63 Sup.: N.51.

B 72 Sup.: I.41; N.27, 67.

B 82 Sup.: N.51.

B 90 Sup.: I.111.

B 108 Sup.: I.25, 27, 33, 76, 94 (2), 95, 99; N.31.

B 113 Sup.: I.5, 18 (2), 22, 38, 43, 49; II.76, 104; N.62.

B 126 Sup.: I.23, 100; II.77.

B 157 Sup.: I.132; II.17, 18 (2), 18-19.

C 4 Sup.: I.49, 68, 96; N.29, 39.

C 69 Sup.: N.47.

C 85 Sup.: I.11, 12, 19, 22, 23, 24 (2), 25, 26 (2), 27(2), 28, 29 (2), 30 (2), 31(2), $33,34,35$.

C 88 Sup.: N.51.

C 89 Sup.: II.109.

C 102 Sup.: II.30; N.30, 34.

C 118 Sup.: I.103.

C 119 Sup.: II.30; N.35, 48.

C 120 Sup.: I.89.

D 13 Sup.: II.55, 71, 73-74.

D 33 Sup.: N.59.

E 6 Sup.: N.41.

E 37 Sup.: N.37, 44, 66, 67, 69.

E 105 Sup.: I.96; N.29.

F 23 Sup.: N.27, 29.

F 88 Sup.: II.67.

F 107 Sup.: N.51.

F 112 Sup.: N.32 (2).

G 69 Sup.: N.61.

G 97 Sup.: I.61, 78 (2), 79 (2), 92. 
H 11 Sup.: N.51.

H 22 Sup.: N.65.

H 43 Sup.: N.44.

H 49 Sup.: II. 28.

L 30 Sup.: II.96, 104.

L 44 Sup.: N.67.

L 110 Sup.: I.4, 11, 18, 20, 21 (2), 22, 23 (2), 24 (2), 26, 28, 29 (2), 33, 34, 35;

II.97, 101, 102, 104; N.65.

L 119 Sup.: II.32 (2); N.48.

M 41 sup.: N.44.

O 50 Sup.: I.87; N.29, 33.

O 117 Sup.: I.125; N.29, 50.

O 123 Sup.: N.69.

P. 32 Sup.: N.39.

P 90 Sup.: N.25, 38, 58.

Q 3 Sup.: I.72, 73, 74, 79, 79-80, 82 (2), 85, 95, 100 (2), 103, 109 (2), 111, 119;

N.36.

Q 13 Sup.: I.5; N.25, 49.

Q 14 Sup.: N.51.

Q 52 Sup.: I.85; N.29.

Q 87 Sup.: I.67, 109; II.80; N.29, 30, 41, 49.

Q94 Sup.: I.90-91, 100; II.23, 40, 98; N.29, 34, 39, 46 (2), 65.

R 20 Sup.: II.65, 79, 109 (2), 110 (2); N.69 (2).

R 111 b: II.68.

R 111 Sup.: N.45, 48, 60.

S 3 Sup.: I.67; II.4 (3), 12, 26, 42, 68; N.29, 30, 42 (3).

S 19 Sup.: II.97, 104.

T 19 Sup.: I.13, 111; N.25, 29, 36.

T 141: II.89.

\& 141 Sup.: N.65.

\& 147 Sup.: II.110.

A 27 Inf.: II.96.

A 57 Inf.: N.47, 52, 66, 67, 69 (2).

A 81 Inf.: I.96; N.29.

A 193 Inf:: N.47, 52, 66, 67, 69 (2).

C 80 Inf.: I.66; N.29.

C 102 Inf.: I.61, 100; N.30, 34.

C 222 Inf.: II.85.

D 293 Inf.: I.89.

D 338 Inf.: II.67.

D 477 Inf.: II.34. 

D 518 Inf.: I.89.
E 10 Inf.: N.51.

H 2 Inf.: N.27, 28, 43, 44, 63, 64.

I 166 Inf.: N.43.

S.Q.E. VIII 13: I.3.

S.Q.E. VIII 14: I.3, 49.

S.Q.J. VII 9: I.3.

S.Q.T. VIII 9: I.4.

\section{Mailand, Bibl. Trivulziana}

685: I.64, 65, 71 (2), 83, 93, 102, 103, 118, 120.

\section{Messina, Bibl. S. Salvatoris}

84: II.6

\section{Messina, Universität}

84: I.148; II.74.

111: I.92.

Mileae, Bibl. Mileens.

78: II.30.

Mitylene, Bibl. gymnas.

1: II.40.

33: II.34, 80.

\section{Modena, Bibl. Estense}

18 (III A 4): II.67.

54 (III B 2): I.38.

61 (III B 9): I.18, 56, 112, 117, 148; II.7, 79, 102, 106.

78 (II C 11): I.96.

85 (III C 6): I.44, 49.

97 (III C 18): I.61.

107 (II D 8): I.96.

109 (II D 10): I.60, 71 (2), 83, 93; II.9, 22.

115 (II D 16): I.40, 128; II.28, 30, 32 (2), 33, 102.

135 (II E 2): II.9, 106.

145 (II E 12): II.9 (2), 22.

151 (II E 18): I.148.

174 (II F 9): II.82 (2).

175 (II F 10): I.65. 
191: I.38.

210 (III G 6): I.111; II.9 (2), 22.

211 (III G 7): I.104.

213: I.73.

213 (III G 9): I.22, 77, 82, 85, 95 (2), 100, 109.

216 (II H 1): I.96, 133.

217 (II H 2): I.76, 100.

218 (II H 3): I.28, 81, 89.

219 (II H 4): I.68.

220 (II H 5): I.4, 20, 23 (2), 24 (2), 25, 26 (2), 27, 29, 33, 34, 35.

226 (II H 11): I.66, 87 (2), 88 (2), 115.

227 (II H 12): I.11 (2), 22, 24, 25, 27 (2), 28, 29-30, 30 (2), 31 (2), 34.

233 (III H 5): I.10, 11, 13, 19, 21, 28, 29.

237 (III G 18): I.69, 70, 78 (2), 79, 80, 84, 103, 107, 130.

240 (III F 17): I.101; II.67; N.34

Montpellier, Bibliothèque de l'école

(Vgl. Montfaucon, II p.1199): II.89.

\section{Moskau, Synodialbibl.}

-: II.43.

?: II.71.

[?]: I.110.

[complures]: I.58.

(Vgl. Bussemaker a. a. O. p. 75): II.20.

51: I.60.

52 (so!) = 464: N.29.

260: I.72; II.74; N.31.

283: I.60; N.29.

292: I.72; N.31.

395: I.77.

436: II.91.

439: I.40, 41.

446: II.11.

464: I.60, 87 (2), 88, 88-89, 92, 93, 111; II.106; N.29.

465: I.71(2), 102.

466: I.60, 61, 63, 64, 65, 70, 71 (2), 79, 86, 92, 93, 96, 103, 108; II.48, 50, 96;

N.29.

467: I.73, $74(2)$.

468: I.100; II.7, 41.

477: II.34. 
498: II.71.

499: II.72.

München, Bibl. August.

542: II.44.

\section{München, Hof- und Staatsbibliothek}

(vgl. Bussemaker a. a. O.): II.20.

[-]: I.149.

[Hardt 1 p. 448]: II.81.

graec.

29: II.67, 71 .

39: I.76, 126, 134, 135; II.63; N.31-32, 38, 39 (2), 58.

69: II.79, 109, 110.

70: II.17, $18(2), 28$.

71: I.4, 5, 8, 10 (2), $11(3), 12,13,18(2), 19,20(3), 21(2), 22(2), 23(3), 24$

(3), $25(2), 26,27(2), 28(2), 29(2), 30(3), 31(3), 32(3), 33(3), 34(3)$,

35 (3), 38, 46, 48, 110; II.37, 93; N.36.

72: I.40, 46; II.17, 18 (2), 19, 37, 58, 72.

100: II.67.

105: I.112; II.41, 44; N.36, 51.

109: I.72, 101, 111; N.31, 34, 36.

227: II.26.

231: I.104; N.35.

236: I.93; II.30; N.34, 48, 49 (2).

276: I.44, 130.

278: I.130; N.39.

287: I.13, 49; II.87; N.51.

288: II.41, $52 ;$ N.54.

308: II.94.

362: II.109.

401: N.53.

419: II.67; N.59.

469: I.95, 96, 102, 111; II.88 (2), 90, 91; N.34, 35, 36, 37, 64, 65.

484 (542) (?): II.33.

494: I.46.

498: II.94.

511: II.66.

514: II.107.

521: N.48.

525: I.13, 136. 
536: II.67.

551: N.38, 39, 56.

562: II.67.

583 (olim Uffenbachianus): N.58.

lat.

807: I.149.

Neapel, Biblioteca Borbonica

I 17: N.60.

II C 33: II.94.

II C 33 (olim 34): II.82.

III C 2 (260): I.44.

III D 15: N.30, 31, 41.

III D 21: N.45.

91 (II C 33): I.13, 35.

187 (II F 30): I.38.

229 (III D 15): I.62; N.30.

304 D III 20: I.96; II.71.

305 (III D 21): II.17, 18 (2), 19.

306 (III D 22): II.77.

307 (III D 23): II.32, 65, 110.

Neapel, Bibl. dei Gerolamini

XXII.1: I.38, 44, 46, 64, 128; II.7, 28, 63.

Neapel, Bibl. di S. Giovanni di Carbonara

15: I.56; II.47.

27: I.38.

42: II.71.

Neapel, Bibl. Oratoriana

152: I.44, 131, 133, 134.

152 (Pil. XXII no. 1): II.28, 63.

Neu-Ephesus, Bibl. Ephes.

-: N.43.

[Norfolk]

[nr. 3139]: I.13. 
Orléans, Bibl. communale 1032: II.47.

[Oxford, Ashmol.]

[mss. Angl. 7638]: I.122.

[mss. Angl. 7787]: I.122.

Oxford, Bibl. Aedis Christi

34: I.56, 149.

81: II.17, 18 (2), 19.

Oxford, Bibl. Bodleiana

- (Puschm. I p. 90): II.12.

e 19 [31.528]: II.30.

[cat. mss. Angl. 1355]: I.61.

[cat. mss. Angl. 1552]: I.61.

[Cat. mss. Angl. n. 2062]: I.117.

[2753]: I.5.

[bibl. Brit. n. 3500]: I.135.

Auct.

F. Inf. II 3: I.13, 18 (2).

T II 3: I.5, 56.

T II 10: I.38.

\section{Baroccian.}

10: I.41.

50: II.4, 107.

51: I.38.

76: I.5.

82: II.67.

88: I.44 (2), 121, 128, 130, 131; II.6, 20 (2), 21, 34, 47, 57, 75, 77, 81.

[88]: I.126.

94: II.67.

111: II.98.

131: I.47, 56, 72, 78, 88, 110, 126; II.9 (2), 63, 75.

150: I.41, 133; II.11, 17, 21, 33, 34, 38, 41, 49 (2), 56, 58 (2), 60, 69, 74, 80, 85, $86,99,100 ;$ N.67.

171: II.69.

173: II.66.

204: I.4, 5, 8, 10, 11 (3), 12, 13, 18 (2), 19, 20 (3), 21 (2), 22 (2), 23 (2), 24 (3), 25 (2), 26 (2), 27 (3), $28(2), 29(2), 30$ (3), 31 (2), 33 (2), 34 (3), 35 (2), $38,66,110$; II.37, 93, 104. 
216: II.33.

220: I.84; II.43.

224: I.43, 134; II.8, 28, 58, 71, 77, 80.

264: I.76.

2304: II.109.

\section{Canonician.}

1: I.40, 47, 125, 130; II.7. 1.

44: I.84, 85 .

56: II.67.

109: II.6; N.43.

\section{Clarkian.}

16 (18378): I.41, 113, 133.

Ger. Langbainii Advers.

2: I.41.

Is. Casauboni Advers.

4: I.72.

27: II.34.

\section{Laudian.}

[cat. mss. Angl. n. 877]: I.133.

58 (nunc 59; Bodl. 708): I.128; II.20, 88.

62 (Bodl. 747): II.110.

[1013]: I.5, 13.

[1252]: I.13.

[1257]: I.5.

[1355]: I.5, 13.

C 54 (nunc 56; Bodl. 706): I.92.

C 55 (nunc 57; Bodl. 707): I.87, 88 (2), 89.

C 57 (nunc 58, Bodl. 709): I.63, 64, 65, 83-84, 85, 89, 91, 115.

C 60 (Bodl. 749): II.6.

Miscell.

-: II.28.

20: I.70, 131; II.21, 40.

69: II.67.

130: I.26, 77-78; II.99.

132: I.5, 13, $18(2), 77$.

162: II.67.

189: II.6.

210: I.38.

211: II.36.

211 (Auct. T II 11): N.50.

212: II.36. 
212 (Auct. T II 12): N.50.

241: II.79.

241. Auct. T IV 3: II.109.

278: II.49, 68 .

d'Orvillian.

X 1. 1. 3: I.101, 110, 111; II.37, 71.

$X$ 1. 4. 3: II.67.

$X$ 1. 4. 29: I.49.

X 2.6. 2 (= 432): II.67.

Roe.

14: II.6, 38.

14 (260): I.41.

14 (Bodl. 260): II.63, 104.

15 (Bodl. 261): I.125; II.10, 63, 102 (2), 109 (2), 110.

15 (Bodl. 260 [261 bei Ackermann]): I.128.

XIV (260): I.13, 34-35.

[Oxford, Coll. Merton.]

[685]: I.63, 91 .

[687]: I.5, 13.

[688]: I.5, 13.

[689]: I.5, 13 .

[722]: I.13.

[729]: I.49.

[Oxford, Coll. Novi]

[1130]: I.13.

[1134]: I.13.

[Oxford, Eccl. Wigorn.]

[745]: I.97-98.

[768]: I.13.

[1760]: I.5.

[Padua, Bibl. Canonicor. Lateranens.]

[-]: I.28; N.26.

[Padua, Bibl. Cathedr.]

[P. 129]: II.17, 18 (2), 19; N.45. 
Padua, Bibl. Joann. Rhodii -: I.10, 13, 19, 61, 63, 64, 66, 68, 85, 89, 93, 96, 98 (2), 104, 119, 134; II.29, 30, 33, 70,97 (2).

Padua, Bibl. Marci Mantuae -: II.30.

Padua, Bibl. S. Joannis in Viridario ad dextr. plut. 16: II.6; N.43. ad dextr. plut. XVI: II.70. ad dextr. plut. XVII: I.3, 5, 13, 21, 30; II.77; N.25 (30, 26 (3).

Padua, Bibl. Tomasini -: II.17, 18 (2), 19.

\section{Palermo, Biblioteca nazionale}

IV H 8: II.9.

XIII C 3: I.13, 42, 123, 128; II.6, 55, 98, 102, 110.

[Paris, Bib.Regia/B. R.]

2 Parisini regii bei Migne: N.46.

[120 ap. Montf. II 902]: I.103.

[349]: II.58.

Paris, Bibl. de la ville de Paris

12: II.33.

58: II.6, 8 .

Paris, Bibl. de Paris

4: I.38.

42: II.67.

57: II.71.

Paris, Bibl. Facult. Méd./Med., Bibl. Facult. Medic./Facult. Méd. (Med.)/ Faculté de Méd./Faculté de Médecine.

-: II.15, 74, 109.

nr. ?: I.150.

14: I.57, 103.

14 (ol. Bibl. de Paris 57): II.74, 89.

76: II.6.

145 (ol. Bibl. de Paris 59): II.71. 
Paris, Bibl. Mazarine 4461: II.33.

\section{Paris, Bibl. Nationale}

\section{Coislin.}

bei Migne: N.46.

[ap. Montf. II 447]: I.64, 65.

[ap. Montf. II 447]: I.128.

[ap. Montf. II 448]: I.96.

[ap. Montfauc. II 448]: I.126.

8: II.77; N.62.

78: II.52.

79: II.52.

115: II.67.

120: II.67.

123, f. A: N.62.

158: II.41, 43, 56.

163: I.103.

168: II.77.

228: I.101; II.40.

229: II. 40 .

235: II.40.

259: II.67.

294: II.67.

300: I.123.

321: I.41.

[321 (?)]: I.41

332: II.9 (2).

333: I.68.

334: I.80, 130; II.109, 110.

335: I.42, 114, 117, 123, 133, 136, 149; II.15, 25, 28, 64, 79, 91.

[335]: I.28.

336: I.72.

384: II.63.

387: I.98.

[1018 (?)]: I.13.

Imprimés

Rés. Te 138.27: I.117; II.33.

\section{Parisin.}

36: I.5, 13, 25, 49; II.29, 80.

396: I.5, 32; II.28, 49. 
476: II.40.

478: II.21.

479: II. 40 .

500: II. 21.

503: II.40.

777 A: II.40.

825: II.67, 101.

826: II.67.

827: II.67.

827 A: II.67.

834: II.35.

835: N.50.

912: II.21, 52.

940: II.40.

956: II.40.

968: II.40.

985: I.43, 68, 80; II.49, 98.

993: II.106.

1000: II.52.

1007: II.40.

1009: II.40.

1010: II.40, 99.

1044: II.67.

1045: II.67.

1046: II.67.

1053: II.40.

[1097 (?)]: I.63.

1247: II.40.

1268: II.9, 67.

1277: II.40.

1297: I.13.

1310: I.126; II.94.

1327: I.38.

1346: II.94.

1389: II.25.

1405: II.44.

1438: I.101.

[1444 (?)]: I.11.

1542: II.40.

1603: II.34, 67.

1630: I.40, 41, 113, 130; II.9, 14, 59, 64, 102 (2). 
1631: II.28.

1644: I.61.

[1667 (?)]: I.46.

1739: II.67.

1760: I.38.

1766: II.94.

1788: II.94.

[1819 (?) = 1849 (?)]: I.66.

1831: I.149.

1848: II.10.

[1848 ?]: I.13.

1849: I.11 (3), 66, 69, 106, 108 (2).

1865: I.149; II.10, 80.

1868: I.4, 11, 18, 21 (2), 22, 28, 29; II.37.

1883: I.5, 13, 46, 60, 61, 63, 76, 80, 114, 131; II.6, 9, 71, 74, 104; N.32, 43.

1884: I.5, 13, 46, 105; II.9, 40.

[1884]: I.38.

[1884 (?)]: I.41.

[1885]: I.38.

[1885 (?)]: I.46.

1893: II.9.

1943: II.22.

1949: I.80.

1991: I.112-113, 135; II.44, 47, 77, 82, 111; N.69.

1995: I.43.

2027: II.35.

2028: II.9.

[2030 (?)]: I.20, 33.

2037: N.58 (2).

2047: I.18, 40; II.9, 77, 106.

2047 A: II.9, 22, 63.

2048: II.9.

2077: II.67.

2091: I.43, 125.

2098: II.109.

[2113 (?)]: I.101.

2118: N.58.

[2123 (?)]: I.98.

[2137 (?)]: I.88.

2139: II.44 (2), 76, 82 (2).

[2139 (?)]: I.113. 
2140: I.4, 5, 8, 10, 11 (3), 12, 13, 18 (2), 19, 20 (3), 21 (2), 22, 23 (2), 24 (3), 25 (2), $26(2), 27$ (3), 28 (2), 29 (2), 30 (3), 31 (2), 33 (2), 34 (2), 35 (3), 38, 110; II.93.

[2140]: I.22.

2141: I.4, 5, 8, 10, 11 (3), 12, 13, 18 (2), 19, 20 (3), 21 (2), 22, 23 (2), 24 (3), 25 (2), $26(2), 27$ (3), 28 (2), 29 (2), 30 (3), 31 (2), 33 (2), 34 (2), 35 (3), 38, $110 ;$ II.37, 93.

[2141]: I.22.

2142: I.1, 4, 5, 8, 10, 11 (3), 12, 13, 18 (2), 19, 20 (3), 21 (2), 22, 23, 24 (3), 25 (2), 26 (2), 27 (3), 28 (2), 29 (2), 30 (3), 31, 32, 33 (2), 34 (2), 35 (2), 38, 110; II.37, 93.

[2142]: I.22, 31.

[2142 (?)]: I.23 (2), 35 .

2143: I.4, 5, 8, 10, 11 (3), 12, 13, 18 (2), 19, 20 (3), 21, 22, 23 (2), 24 (3), 25 (2), $26(2), 27$ (3), 28 (2), 29 (2), 30 (3), 31 (2), 33 (2), 34 (2), 35 (3), 38, 110; II. $37,93$.

[2143]: I.22.

[2143 (?)]: I.21.

2144: I.4, 5, 8, 10, 11 (3), 12, 13, 18 (2), 19, 20 (3), 21 (2), 22, 23 (2), 24 (3), 25 (2), $26(2), 27$ (3), 28 (3), 29 (2), 30 (3), 31 (2), 33 (2), 34 (2), 35 (3), 38, 110 ; II.37, 93, 111.

[2144]: I.22.

2145: I.4, 5, 8, 10, 11 (3), 12, 13, 18 (2), 19, 20 (3), 21, 22, 23 (2), 24 (3), 25 (2), 26 (2), 27 (3), 28 (2), 29 (2), 30 (3), 31 (2), 33 (2), 34 (2), 35 (3), 38, 110; II.37, 93.

[2145]: I.22.

2146: I.4, 5, 8, 10 (2), 11 (3), 13, 18 (2), 19, 21, 27 (2), 28 (2), 29, 30 (3), 31 (3), $32(3), 33,34,35,38,40$.

[2146]: I.22, 30 .

[2146 (?)]: I.23, 34, 35 .

2147: I.13, 21, 22, 28, 29, 150; II.98.

[2147]: I.22.

[2147 (?)]: I.28.

2148: I.8, 18 (2), 21, 23 (2), 24, 25 (2), 26, 27, 29, 33, 39, 68, 96; II.37.

[2148]: I.13. 24 (2), 26, 34, 35 .

2149: I.13, 56, 115, 149; II.37, 69, 74, 77, 104.

2149 (?): I. 24.

2150: I.13; II.26.

2151: I.117; II.33, 37, 71, 89 (2), 97.

[2151 (?)]: I.111.

2152: II.66, 91. 
2153: I.88, 101, 118; II.7, 79 (2), 80, 92, 109 (2), 110.

2154: I.68; II.62; N.58.

[2154]: I.13.

2155: I.33, 68, 80, 89, 98, 131, 149; II.40, 101.

2156: I.80, 96, 98, 101, 111, 126.

[2156 (?)]: I.93

2157: I.78, 79 (2), 80, 85, 92, 93, 96.

[2157 (?)]: I.80, 87, 88, 89.

2158: I.80, 96, 98, 101, 111, 126.

[2158 (?)]: I.88.

2159: I.96.

2160: I.92, 101.

[2160 (?)]: I.96.

2161: I.13, 85, 87, 88 (2), 89, 105.

2162: I.92.

2163: I.61.

2164: I.59, 67 (2), 68, 69, 70, $74(2), 76,77,83,98,99$ (2), 106, 110, 111, 133;

N.36.

2165: I.10, 19, 68, 70, 78, 79, 80, 82, 84, 95 (2), 102, 104, 109, 112, 113.

2166: I.20, 73, 82, 84, 93, 95, 103.

2167: I.76, 78, 79, 80, 87, 88 (2), 89, 101, 111, 130.

[2167 (?)]: I.98.

2168: I.5, 13, 103, 105, 107.

2169: I.61, 78, $79(2), 80,92$.

[2169 (?)]: I.59, 101 .

2170: I.82, 96 .

[2170 (?)]: I.81, 82 .

2171: I.92, 101.

2172: I.75.

2173: I.76, 98 .

2174: I.10, 104 .

2175: I.101, 111.

[2175 (?)]: I.61.

2176: I.134.

2177: I.103; II.37, 72.

2178: I.13, 44, 80, 126, 131; II.13, 80, 106.

2179: II.30.

2180: II.30, 45 (2).

2181: II.33, 97.

2182: II.30, 32 (2), 33.

2183: I.122; II.25, 29, 30, 32 (2), 33, 86. 
2184: II.30, 32 (2), 33, 101.

2185: II.29, 30, 32 (2), 33.

2186: II.17, 18 (2), 19.

2187: II.17, 18 (2), 19.

2188: II.71.

2189: II.71.

2190: II.71.

2191: II.6, 8, 77.

2192: II.6, 8, 77; N.43.

2193: II.6, 8, 90; N.65.

2194: II.6, 24, 38; N.51, 63.

2195: I.99; II.6.

2196: II.6; N.43.

2197: II.6.

2198: II.6.

2199: II.6.

2200: II.11.

2201: II.11.

2202: II.11, 17, 18 (2), 19.

2203: II.11.

2204: II.11, 77, 102.

2205: II.77.

2206: II.17.

2207: I.86; II.77.

2208: II.3, 74, 77.

[2208 (?)]: I.114.

2209: II.77.

2210: I.121, 149; II.26, 50, 76, 77.

2211: II.77.

2212: II.77.

2213: II.77.

2214: II.77.

2215: II.77.

2216: II.77.

2217: II.3, 77-78.

2218: II.75, 98.

2219: I.5, 13, 67, 105, 109; II.101.

2220: II.17, 18, 89, 96, 99, 101, 102.

2222: I.13; II.63, 97, 104.

2223: I.13; II.63, 97, 104.

2224: I.13, 44, 125, 128; II.30, 32 (2), 52, 63, 69, 85; N.38. 
2225: II.63, 67.

2226: II.63.

2227: II.63.

2228: I.5, 13, 103, 112, 128, 129; II.6, 34, 36, 75, 83, 92, 98, 104, 106.

2229: I.5, 10, 13, 40, 89, 131; II.28, 96, 99, 101, 102.

[2229 (?)]: I.19, 41 .

2230: I.118; II.6, 9; N.37.

[2230]: I.126, 133.

2232: I.92; II.109 (2).

[2232]: I.26.

2233: II.110.

2234: II.18, 19, 109.

2235: II.110.

2236: II.41, 52, 111.

2237: I.149; II.6, 7, 69, 71, 74 .

2238: I.114; II.33, 69.

2240: I.41, 75; II.8, 23, 64, 65, 74, 79, 91; N.59.

[2242 (?)]: I.30.

2243: II.33, 69, 74; N.53 (2).

2244: II.28, 57.

[2245 (?)]: I.107.

2246: I.80, 89, 91, 101.

2247: I.10, 11 (3), 12, 49, 106; II.15, 37, 71, 89.

[2247]: I.34.

[2247 (?)]: I.107.

2248: I.10, 11 (3), 12, 106; II.15, 37, 71, 89.

2248 (?): I.107.

[2248]: I.34.

2249: II.111, 112 (3).

[2250]: I.13.

2251: II.112.

2252: I.111; II.112.

[2252 (?)]: I.111.

2253: I.4, 5, 8, 10, 20, 21 (2), 22 (2), 23, 33, 38, 40, 68; II.113.

[2253]: I.22.

[2253 (?)]: I.20, 33 .

2254: I.8, 11 (2), 12, 19, 20 (2), 21, 22, 24 (2), 25, 27 (2), 28, 30 (3), 31 (2), 33, $34,38,57$; II.37.

[2254]: I.4. 5.

[2254 (?)]: I.10, 12, 18 (2), 20, 21, 22, 23 (3), 24, 25, 26 (2), 27, 28, 29 (2), 31, $32(3), 34,35(4)$. 
2255: I.4 (2), 5, 8, 10, 11, 12, 13, 18 (2), 20, 21 (2), 22, 23 (2), $24(2), 25,26$

(2), 27, 28, 29 (2), 31, 32 (3), 33(2), 34 (2), 35 (2), 38, 40, 57, 110; II.37, 93.

[2255]: I.22.

[2255 (?)]: I.23, 35 .

2256: I.5, 13, 28, 30, 117; II.6, 7, 43, 45 (3), 68, 109 (2), 110.

2257: I.5, 13, 105, 107; II.63, 101, 102.

[2257 (?)]: I.31.

2258: I.13.

2259: I.13.

2260: I.13, 41, 80, 86, 93, 128, 133; II.30, 60, 96, 99, 109, 110.

2261: II.71, 89 .

2262: II.71, 89 .

[2262 (?)]: I.92.

2263: II.71, 89.

2264: II.89.

2265: I.61, 93.

2266: I.5, 13, 105, 107.

[2266 (?)]: I.20, 33.

2267: I.63, 64, 65, 77, 80; II.98.

2268: I.13, 105.

2269: I.5, 44, 49, 68, 73, 78, 80, 81, 82, 83, 93, 95, 96, 109, $112(2), 128,129$, 130, 134; II.75.

[2269]: I.56.

[2269 (?)]: I.20, 33, 82, 113.

2270: I.61, 68, 73, 81, 82, 96, 101, 118, 129; II.6, 109.

[2270 (?)]: I.93.

2271: I.61, 68, 73, 81, 82, 96, 101.

2272: I.80, 89, 91 .

[2272 (?)]: I.81. 82 .

2273: I.61.

2274: I.92.

2275: II.24, 100, 115.

2276: I.78, 80, 86, 101, 102; II.17, 83, 98.

[2276 (?)]: I.87, 88 (2), 89.

2277: I.61, 74 .

2278: I.13, 69, 105.

[2278 (?)]: I.61, 74 .

2279: I.70, 96.

2280: I.92, 101.

[2280 (?)]: I.96. 
2281: I.68.

2282: I.101, 111.

2283: I.60, 63, 64, 68, 71 (2), 73, 74, 75, 78, 79 (2), 80 (2), 81, 82, 83, 84, 89, $91,92,109,112,130$.

2284: I.80.

2285: I.61.

2286: I, 133; II.17, 20, 25, 29, 33, 34, 54, 55 (2), 68, 70, 107; N.55 (2), 60, 68. 2287: I.24, 110; II.30. [2287 (?)]: I.11.

2288: II.17, $18(2), 19,88$.

2289: II.17, 18 (2).

2290: II.108.

2291: II.108.

2292: II.78.

2293: II.78.

2294: I.41, 44, 114, 128, 133; II.34, 78, 80.

2295: II.67, 76.

2296: I.13; II.96, 104.

2297: II.101.

2298: II.99.

2299: II.21, 62, 63 (2), 67.

2300: II.63.

2301: I.40; II.28.

[2301 (?)]: I.40.

2304: I.44, 93; II.109 (2), 110.

[2304 (?)]: I.61, 101.

2305: II.109 (2), 110.

2306: I.61, 101; II.102 (2), 109 (2).

2307: I.61, 101; II.65, 102 (2), 109 (2), 110 (2).

2308: I.44, 93, 113, 120; II.102, 109 (2); N.37 (2).

2309: I.128.

2312: I.114.

2313: I.133; II.34, 80.

2314: II.14, 49 .

2315: I.40, 89, 113, 114; II.51, 64, 65, 69, 79; N.59.

2316: I.5, 13, 21, 43, 47, 78, 113, 125; II.13, 40, 54, 60, 80, 102.

2317: I.21, 63-64, 131, 136.

2318: I.114; II.79.

[2319 (?)]: I.114.

2320: I.28, 43, 44, 49, 117, 121, 131, 132, 134; II.20, 24, 80.

2321: II.71, 89 . 
2324: I.123; II.36, 48, 60 .

2325: II.100, 112 (2), 115.

2326: II.115.

2327: II.24, 43, 100, 111, 112 (2), 115 (2).

2329: II.112 (2).

2330: I.5, 13, 28, 44, 49, 128.

2331: II. 88,90 .

2332: I.4, 5, 8, 64, 73, 78, 79 (2), 80, 85, 89 (2), 91, 100, 102, 105, 119, 131;

II.60.

[2332]: I.10, 11, 12(20, 19. 20 (3),, 21, 22, 23 (2), 24 (3), 25 (2), 26, 27 (2), 28, 29 (2), 30 (2), 31 (2), 33 (2), $34(2), 35$ (3), 38, 61, 64.

2333: I.61.

2334: II.17, $18(2), 19$.

2335: II.72.

2336: II.55.

2337: II.34, 74, 80 .

2381: II.3, 9 .

2383: I.107, 129, 150.

2408: II.61, 74 .

2419: I.42, 43; II.43, 45, 75 .

2422: II.107.

2426: II.35, 44, 82 (2), 87, 88.

2428: II.67.

2494: I.130.

2502: II. 43,45 (3).

2506: N.53.

2509: II.35.

2510: I.42, 48, 114, 121, 133, 149; II.34, 58, 71, 74, 80, 106.

2537: II. $43,56$.

2540: II.10.

2545 (? = 2145 ? cf. Kühn 1.1): I.5.

[2545 (?) = 2145 ?]: I.4, $11(2), 12,13$.

[2545 (? = 2145 ?)]: I. 8,18 (2), 20 (3), 21 (2), 22, 23, 24, 25, 26 (2), 29 (2),

$30(2), 31(2), 33(2), 34(2), 35(3)$.

[2546 (? = 2146 ?)]: I.27, 28 .

[2548 (?)]: I.30.

2596: I.13, 18; II.93.

2614: II.37.

2615: II. 37, 59 .

2633: II.61.

2651: II.37. 
2652: I.38.

2664: I.99.

2665: II.36; N.50.

2671: I.41.

[2671 (?)]: I.13.

[2682 (?)]: II.17, 18 (2), 19.

[2699 (?)]: I.41.

2720: II.36; N.44.

2726: II.8.

2731: II.36; N.44.

2755: I.38.

2760: I.111.

[2828 (? = 2228 ?)]: I.5.

2830: I.86; II.36; N.44.

2847: I.56; II, 7, 28.

2894: I.40; II.57, 94.

3023: II.94.

3035: I.89, 110, 113, 131.

3044: II.9.

3047: I.38.

3050: I.38.

3052: I.38.

3076: II.17, 74 .

3099: II.71.

[3140 (?)]: I.13.

[3170 (?)]: I.5.

Suppl.gr.

2: I.103.

3: II.34.

35: I.60, 70, 72, 73, 74, 81 (2), 83, 95, 96, 99 (3), 110, 111 (2), 112, 113.

49: II.63.

64: I.13; II.104.

84: II.63.

86: II.67.

148: II.67.

165: I.40; II.11.

194: II.67.

205: I.38.

263: II.56.

270: II.15.

292: I.149. 
338: II.78

341: II.40.

352: I.41.

445: II.17, $18(2), 19$.

446: I.5, 13, 41, 80, 86, 89, 93, 111, 113 (2), 128, 130, 151; II.57, 71, 74, 78, 82, $98 ;$ N.56.

447: I.13, 22, 101, 105. See N.34, 35.

493: II.30, 34 .

494: II.78.

496: I.42, 61 .

608: I.18.

629: I.89, 131; II.59, 88, 89, 93, 99, 101, 102 (2), 106.

630: II.6.

631: II.6.

632: II.6.

633: II.17.

634: I.60, 61, 64 (2), 67, 76, 77, 83, 92, 93, 113, 119, 125, 128, 133, 150.

635: I.119, 120; N.32.

636: I.100; II.37, 48.

637: I.41; II.7, 55, 63, 90, $102(2)$.

638: II.53.

639: II.107.

640: II.67.

641: II.67.

652: II. 44 .

654: II.65.

662: II.61.

675: I.41.

681: I.117.

682: I.13, 114; II.26.

683: I.149; II.7, 60, 74.

684: I.42, 100, 123.

688: II.93.

702: II.67.

727: I.67, 120.

728 (Minas IZ): I.47.

754 (Minas): I.47.

755: II.23.

764: I.76, 93, 149; II.11.

765: I.67.

793: I.150. 
836: II.10.

924: II.80.

1022: II.100, 115.

1098: II.30.

1156: II.78; N.62.

1193: I.99, 114 ; II.79.

1194: I.56, 149.

1197: II.30.

1202: I.149; II.17, 34, 60, 74, 80, 86, 92.

1240: II.6.

1254: I.47.

1297: I.114; II.11, 79, 93.

Parma, Bibl. Palatina

Fondo De-Rossiano

7: II.28.

Patmos, Bibl. Monast. St Ioann.Evang.

-: II.67; N.43.

11 (bei Coxe, Mss. gr. of the Levant): N.59.

71 bei Coxe, Mss. gr. of the Levant: N.62.

KI': N.51.

111: I.78.

277: II.6.

Petersburg, Kaiserl. öffentl. Bibl.

116: I.123.

164: II.88, 89, 90.

Pistoja, Biblioteca Fabroniana

141 (308): I.13, 105, 107.

\section{Raudnitz}

11: II.6.

Raudnitz, Bibl. Ducis Lobcovic VI F C 37: II.6.

Ravenna, Biblioteca Classens.

70: I.58.

1312 H: I.61, 101. 
[Reims]

[548]: I.21.

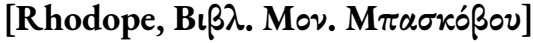

[548]: I.3.

Rodosto

- : I.117.

Rom, Bibl. Angelica

4 (C.4.16): I.39, 40, 46; II.15, 37, 58 (2), 81; N.56.

17 (C.5. 4): I.116, 133; II.44, 52, 58, 64, 74, 78, 80, 85, 102.

68 (C.2.7): II.79.

80 (C.1. 11): I.13.

Rom, Bibl. Barberin.

I 5: I.4, 13 .

I 11 (= 136): I.13.

I 17: N.48.

I 49: II.7.

I 49 (= 359): II.92.

I 72 (440): II.81.

I 80 (273): I.67, 121.

I 91 (382): II.88, 90; N.64.

I 118: I.67; II.33, 71 .

I 127: I.26, 39, 40, 56, 101, 113, 117, 118, 128, 131; II.10, 15, 28, 62, 76, 80, 87, 102 (2).

I 152: I.121.

II 58: II.32, 62; N.57.

III 63 (= 245): I.42, 123; II.57, 64 .

V 18: II.67.

VI 5: II.67.

136 (= I 11): I.5.

276: I.3.

382 (I 91): II.88; N.64.

Rom, Bibl. Corsiniana (= Acc. d. Lincei)

14 (Rossi 358): II.29.

1410 (36. E. 26): N.26, 28 (2), 39 (2). 
Rom, Bibl. St. Petri

H 45: I. 58 .

\section{Rom, Bibl. Vallicellana}

- (Allatianus): II.68.

15 (B 70): II.11.

21 (B 93): I.72.

78 (F 9): II.37.

1106 (F 83): I.26.

\section{Rom, Bibl. Vaticana}

\section{Ottobon.}

89: II.11.

129: II.71, 72.

145: II.63.

150: I.13.

157 A: I.13.

157 B: I.5, 13.

192: I.5; II.94.

235: II.71, 89.

259: II.36.

275: II.67.

311: I.64, 65, 80, 111; II.6, 98, 100.

441: II.20.

464: II.67.

Palat.

13: II.94-95.

31: I.96.

48: II.30; N.45, 49 (2).

54: I.80, 85, 93, 98.

77: II.30, 32 (2), 34, 68.

126: I.56.

128: I.13, 61, 105 .

129: I.56.

132: I.38.

143: I.5, 13, 42, 44, 81, 110, 112, 113, 124, 133, 149.

155: I.41.

157: I.80, 89, 91, 107, 130; N.35.

173: I.107.

192: I.4, 5, 8, 10 (2), $11(2), 12,13,18,19,21,23(2), 26,27(2), 28(2), 29(2)$, 30 (3), $31(3), 32(3), 33,34,35,38,44,46,48$. 
199: I.5, 13, 61, 76, 95, 96, 149; II.6, 9; N.25 (2), 29, 30, 32 (2), 36, 37, 41, 43, $60,61,65,68$.

226: II.43.

251: I.68.

278: I.105.

279: I.13, 89, 114, 125, 133, 134; II.7, 60, 80.

295: I.89, 126.

297: I.13, 48, 60, 111, 130, 149; II.75.

302: II.89.

328: II.95.

358: II.7.

363: I.47.

370: II.67, 110.

375: II.71.

385: I.105; II.67.

398: I.38.

400: I.46, 48, 56, 80, 86, 130, 133, 134; II.32, 48, 49 (2), 63, 80; N.49 (2).

428: I.57, 128, 150; II.10, 28.

\section{Passioneian.}

2: I.68.

\section{Reg. Suec.}

154: I.61, 72; N.31.

172: I.98 (2), 115.

173: $\mathrm{I} .75,77,99,101(2), 102 ;$ N.34 (2).

174: I.92; N.33.

175: I.69, 70, 84, 103, 107; N.35.

176: II.75, 78 .

181: I.40, 86, 113; II.110.

182: I.13-14, 18, 22, 23, 25, 26, 28, 29 (2), 40, 44, 46, 47; II.53.

\section{Urbin.}

64: I.5, 14, 18, 21, 22, 38, 49, 56, 104; II.76; N.35, 61.

65: I.105.

66: II.30, $32(2), 33,39(3)$.

67: I.59, 96, 98, 100, 101, 103; N.54, 66.

68: I.4, 5, 8, 10, 11 (2), 12 (2), 14, 18 (2), 19, 20 (3), 21 (2), 22 (2), 23, 24 (3), 25 (2), $26(2), 27$ (3), 28 (2), 29 (2), 30 (3), 31 (2), 33 (2), 34 (2), 35 (3), $38,48(2), 110,124 ;$ II.37, 93.

69: I.68.

70: I.73, 76, 94 (2), 95, 99, 114; II.7.

158: II.80. 


\section{Vatic.}

$$
\text { [-]: II.41. }
$$

- (Puschm. I p. 89): II.12.

? (Vgl. Daremb. I p. LVIII und 124): II.108.

[apud Montfauc. p. 28]: I.135.

[ap. Montfaucon p. 34]: I.121.

bei Migne: N.46.

12: II.89 (2), 91, 95.

154: I.72; N.31.

178: II.17.

[216]: I.22, 34 .

269: I.115.

276: I.4, 5, 8, 10 (2), 11 (2), 12, 14, 18 (2), 19, 21, 23, 27 (2), 28 (2), 29, 30 (3), $31(3), 32(3), 33,34,38,57$.

277: I.4, 5, 8, 10, 11 (2), 12 (2), 14, 18 (2), 19, 20 (3), 21 (2), 22, 23 (2), 24 (3), 25 (2), 26 (2), 27 (3), 28 (2), 29 (2), 30 (3), 31 (2), 33 (2), 34 (2), 35 (3), $38,57,110$; II.37, 93.

278: I.4, 5, 8, 10, 11 (2), 12 (2), 14, 18 (2), 19, 20 (3), 21 (2), 22, 23 (2), 24 (3), $25(2), 26(2), 27(3), 28(2), 29(2), 30(3), 31(2), 33(2), 34(2), 35$ (3), 38,48 ; II.93.

279: I.14, 105, 114, 117, 134; II.59, 60 (2), 80.

280: I.14, 105, 113, 128; II.50, 60, 63, 88, 102.

281: I.87, $88(2), 89$.

282: I.64 (2), 65, 84, 89, 91; II.6, 55; N.30, 33, 55.

283: I.5, 20, 33, 78, 83, 95, 105, 109, 112; II.75.

284: II.30, 32, 34, 85; N.34, 43, 48, 49 (2), 64.

285: I.47, 61, 68 (2), 73, 78, 80, 81 (2), 82, 96, 101; II.49, 98.

286: II.17, $18(2), 19$.

287: II.71.

288: II.71.

289: II.30; N.49(2).

290: II.32; N.49.

291: II.88, 89; N.64.

293: I.41; II.55.

294: I.5, 8, 14, 117; II.80.

295: II.15, 78.

296: I.114; II.78.

297: II.6; N.43.

298: II.6, 8; N.43.

299: II.4 (2), 7, 26, 27, 33, 38, 42, 65, 68, 78, 109, 110; N.42 (2), 43, 56, 57, 59,62 . 
300: I.91 II.40.

423: II.67.

429: II.100, 109.

489: II.67.

517: II.108.

578: II.75.

671: II.54.

690: I.41.

695: I.135; II.35.

854: II.95.

855: II.95.

876: I.41; II.74.

952: II.43.

1028: I.92.

1052: II.108.

1062: II.104.

1063: I.78, 107, 109, 112; N.35.

1064: I.87, 88 (2), 89.

1065: I.41.

1066: I.40, 96, 113; II.43; N.36, 52.

1095: I.41.

1133 (1134 Kl.): I.49; II.37.

1174: II.24, 27, 41, 95, 96, 100, 112; N.48.

1191: II.108.

1194: II.108.

1276: I.40.

1277: II.95.

1309: I.38.

1343: II.20.

1347: II.93.

1354: I.38.

1427: II.71, 72.

1444: I.113, 135; II.44, 76, 101.

1467: I.38.

1470: II.25.

1595: I.114.

1614: I.111; II.9, 22.

1692 B: I.113; II.10, 44.

1695: II.63.

1700: I.125.

1759: I.28. 
1835: II.71.

1845: I.61, 68, 73, 81 (2), 82, 96, 100, 101.

1858: I.107.

1879: II.93.

1885: II. $42,71,91$.

1898: I.114, 115 .

1902: II.26.

1904: II.6, 28, 33; N.43.

1911: II.6; N.43.

1949: II.8.

2154: II.26.

2182: I.93; II.30, 109, 110.

2202: II.6; N.43.

2238: I.18.

2250: II.58.

2254: I.5, 93; II.104.

2256: I.114, 128, 133; II.91.

2259: II.24, 95 (2).

2304 [olim 2217]: I.18; II.7, 27, 89, 101.

3062: N.43.

3073: N.43.

4423: I.111.

7152: II.65.

Vatic. lat.

5763: I.76.

\section{Rom, Fonds Colonna}

12: II.89.

Rosanbo, Bibl. de M. le marquis de Ros.

286: I.98.

Salamanca, Bibl. Univ.

1.1.11: II.71.

1. 1. 14: II.78.

(Epistt. septem): II. 70.

Saloniki, Bibl. gymnas.

17: II.40. 
Sáros-Patak, Bibl. d. reform. Colleg. -: II.43.

Sens, Bibl. communale

nr. 209: I.38.

Miscell. 187: I.5.

Toledo, Bibl. del cab. de la igl. Catedr.

101, 15: II.6, 41.

Tübingen, Univ.-Bibl.

2: II.95.

23: I.20.

37: I.20.

Turin, Bibl. Nazionale (Universitaria)

?: II.71.

(« zitiert bei Montfaucon, 1837, 'Gal. de IV elementis'; nicht verifiziert »): I.64.

6 b IV 6: I.126; II.63.

6 b IV 6 (B I 6): I.76, 134.

8 B V 31 (B VI 29): I.111.

17 B VII 22 (B I 12): I.48, 49, 56, 96, 132, 134, 149; II.41, 58, 64.

156 b II 10: I.5; II.63, 110.

177 b II 31: I.41.

179 b II 33: I.56.

287 c I 42: II. 28.

333 b I 1: II.59, 74, 89, 93.

B V 33 (179 b II 33): I.14.

C VI 3 (287 c I 42): I.40, 47.

C VI 21 (b VI 18): I.44; II.82, 87; N.53.

Upsala, Upsal. bibl. acad.

8 (e don. Jo. Gabr, Sparfwenf. 49): I.38, 117; II.28, 78.

nr. 25: I.73.

30: II.63.

34: II.104.

Urbini et Pisauri, Bibl. Ducis

-: II.40. 


\section{Venedig}

cod. Venetus: I.60.

Venedig, Bibl. Andreae Asulani

Vorlage d. Aldina 1525: I.59.

Venedig, Bibl. Andreae de Rubeis

(gr.?): I.67.

Venedig, Bibl. Josephi de Aromatariis

-: I.67 (2), 70 (2), 84, 109, 113, 115, 119, 125, 133; II.17, 18 (2), 19, 49, 96, 101.

[Venedig, Mon. St. Michael.]

[-]: I.64.

Venedig, Bibl. monast. St. Michael.

104: I.14.

Venedig, Monast. S. Michaelis prope Murianum

93: I.92.

Venedig, Bibl. monaster. St. Michaelis Venet. propre Murianum 132: I.74.

Venedig, Bibl. Nazionale Marciana

graeci

[-]: I.20 (2).

[-]: I.124.

[?]: I.10.

[?]: I.33.

[ap. Montf. I 472]: I.10.

92: II.25, 30, 32, 79.

173: II.95.

257: II.9, 10, 22.

266: II.67.

269: I.4, 5, 8, 10, 11 (2), 12 (2), 14, 18 (2), 19, 20, 21 (2), 22 (2), 23 (2), 24 (2),

25, $26(2), 27(3), 28(2), 29(2), 30(3), 31(2), 33,34(2), 35(3), 38,57$, 110; II.93.

270: II.17, $18(2), 19$.

271: II. $25,30,32(2)$.

271 (jetzt 727): N.49(2). 
272: II.30, 32 (2).

272 (jetzt 728): N49 (2).

273: II.30.

275: I.61, 64 (2), 65, 78, 79 (2), 80.

276: I.70, 73, 74, 75, 83, 92, 129; N.33.

[276]: I.114.

277: I.101, 105 .

278: I.101, 105 .

279: I.66, 67, 69, 73, 76, 94, 95, 99, 106, 108 (2), 114; II.7, 86, 91.

[279 (?)]: I.67.

280: I.85, 93, 98.

281: I.70, 72, 73 (2), 81, 83, 86, 99 (3), 100, 102, 103, 112; II.14.

282: I.28, 60, 71 (2), 73, 74, 75, 80, 81, 82, 83, 84, 89, 91, 96, 101 (2), 107, 113.

[282]: I.114.

283: 1.104 .

284: I.73, $74(2), 92$; N.33.

[284]: I.114.

285: I.77, 98, 101, 104, 105.

286: I.96.

287: I.68, 87, $88(2), 89$.

288: I.98, 115; N.34.

289: II.6; N.43.

290: II.6.

291: II.6, 8; N.43.

292: II.78; N.62.

293: II.74, 78 .

294: I.71, 72 .

295: I.114; II.4, 11, 79; N.37, 42, 61, 62.

296: I.8; II.109.

297: II.63.

298: II.109, 110.

299: II.95, 111, 112 (2), 115.

334: N.52.

335: II.82; N.53.

336: I.113; II.44, 82.

480: II.8.

509: I.149.

510: II.109.

529: II.109.

596: II.6; N.43. 
597: II.30, $32(2)$.

609: I.38.

appendix graeca

II, 171: I.95, 134; II.15, 91.

IV, 36 (olim Nanian. 258): II.43.

V, 1: II.78.

V, 2: II.30.

V, 3: II.30.

V, 4: I.59, 64, 66, 68, 70, 71, $74(2), 78,79$ (2), 80, 89, 91, 92, 93, 95, 102, 106 (2), 110, 111, 115, 121; II.59.

V, 5: I.61, 68, 69, 70, 73, 78, 80, 81 (2), 82 (2), 84, 85, 95 (2), 96, 102, 103, $104,105,107,109,112,113,128,130$.

V, 6: I.96.

V, 7: I.98.

V, 8: I.89, 91; II.22, 100, 102, 110.

V, 9: I.29, 60, 68, 68-69, 73, 81 (2), 82, 96 (2), 101, 102, 105, 120; II.11, 12;

N.30, 34, 36, 41 .

V, 10: I.101, 105, 149; II.9.

V, 11: I.76.

V, 12: I.69, 79; II.101, $102(2)$.

V, 12 (olim Nanian. 246): II.101.

V, 13: I.49, 149; II.7, 41, 45, 49, 65, 68, 80, 86, 109; N.53.

V, 13 (olim Nanian. 247): II.43.

V, 14: I.34.

V, 14 (olim Nanian. 248): I.4, 26, 28, 31, 35.

V, 15: I.104; II.37; N.35.

V, 16: I.40 (2); II.7, 9, 28, 34, 80, 96.

V, 18: I.149.

V, 19: I.21, 149 .

V, 20: I.149.

V, 21: I.149.

V, 22: I.149.

XI, 5: I.91.

[Venedig, Bibl. S. Anton.]

-: I.14, 20.

\section{Verona, Bibl. Capitolare}

CXXIX (118): II.63.

57: II.108. 
Vicenza, Bibl. Bertoliana

44 (I.6.15): II.71, 89 .

Warschau, Bibl. Zamoyski

142: I.61.

Wien

- (bei Berth.-Ruelle, Alchim. a. a. O.): II.100.

Wien, Bibl. Colleg. S. J. Rossianus

XI.167: N.27 (2), 28, 30, 39, 51, 56 (2), 63 (2), 65, 66.

Wien, Gollob Privatbesitz

Vindob.: N.27.

Wien, Hofbibliothek

[ap. Lamb. II, c. 6]: I.98.

[ap. Lambec. I.181]: I.65, 83, 89.

[ap. Lambec.-Kollar I p. 273]: I.124.

[ap. Nessel III 27 (?) no. 5]: I.80.

[ap. Nessel III 38 (?)]: I.80.

[ap. Nessel. P. III]: I.102.

[Lamb. VI 85]: I.8.

[Lambec. VI p. 93]: I.149.

[Lambec. VI p. 100]: I.149.

[Lambec. VI p. 151]: I.149.

[Lambec. VI p. 153]: I.149.

[Lambec. ed. Kollar I p. 272]: I.123.

[Lambec. ed. Kollar I p. 275]: I.149.

[v. Lambec. VI 145]: I.42.

69 B 101: I.3.

131: I.79.

[131]: I.126.

[179]: I.44.

hist./histor.

94: II.20.

130: I.14.

lat.

16: II.30.

4772 (tab. Cod. III 382): I.14, 18. 
med.

1: I.96, 117; II.25, 30, 38, 39 (3).

3: II.109.

4: I.23 (2), 24, 25, 26 (2), 27 (2), 30.

5: II. 25,39 .

6: II.6, 25.

8: I.41, 61; II.15, 55, 63, 78, 79 (2), 82, 90, $102(2)$.

[8]: I.56.

10: II.10.

11: II.28, 33.

12: II.6; N.43, 62.

13: I. $28,42,43,93,149$.

14: I.31, 42; II.30, 31.

15: I.119, 149; II.60; N.57.

[15]: I.56; II.10.

16: I.49, 58, 111; II.30, 60, 97, 98, 104, 105; N.57.

16 (ol. 35): I.5; II.71.

18: I.85, 93; II.33, 97.

[19 (?)]: I.128.

20: I.114, 133.

21: II.79, 109 (2), 110.

22: I.85; II.28, 110.

23: II.44, 45 (2), 47-48, 48, 79, 110.

24: I.24, 38 .

25: I.96; II.79.

26: I.114; II.79, 109, 110.

27: II.28, 36, 37, 45, 74, 79, 91.

28: I.14, 89, 92, 134, 136; II.6, 30, 33, 34, 49, 58, 63, 80, $105,106$.

[28 (?)]: I.20, 114.

29: I.134; II.30, 35, 36, 79, 80, 102.

30: I.78, 129; II.75.

31: I.14; II.37, 58, 69, 81; N.39, 51, 56 (2), 63, 66.

32: I.39, 114, 125; II.28, 33, 79.

34: I.101, 105; N.34, 35.

35: I.96.

37: I.149; II.48, 102.

38c: II.37.

39: II.33, 66.

40: I.40; II.79.

41: I.46; II.81; N.27 (2), 28, 39, 51, 56 (2), 63, 65, 66.

[41]: I.56. 
42: II.71.

43: II.37, 93.

44: I.5, 107; II.9, 97, 98, 104, 109; N.35.

45: II.63.

47: I.24, 123, 128.

48: I.14, 96, 99; II.78, 88.

49: I.14; II.87.

50: II.43.

51: II.6.

52: I.40, 41, 56, 86; II.6, 33, 49 (2), 105.

53: I.39, 42, 125, 149; II.64.

[53]: I.56.

phil.

34: II.67.

37: II.82.

74: II.104.

82: I.38.

108: II.82 (2).

178: I.41, 42, 128; II.20, 79; N.39, 68.

179: I.14.

181: II.67.

192: I.26.

219: I.18.

290: II.35.

303: I.72; II.89.

philos. et philol.

179: II.114.

suppl.

8: II.6.

28 (olim Neapolitan.): II.30.

81: II.6.

theol.

68: II.67.

128: II. 35 .

199: II.67.

207: II.95.

293: I.40.

Wolfenbüttel, Bibl. Guelf. August./Bibl. Guelf.

[August. 18, 1]: I.38. 
Guelferb.

837 (757 Helmst.): N.31.

3132 (18.1 Aug. $\left.4^{\circ}\right)$ : N.27.

4148 (64 Weissenb.): N.32.

Guelferbyt. Ebert

2 (47 Gud. gr.): N.62.

2 (Gud. 47): II.78.

364 (Gud. 7 et 18): I.58.

364 (7 und 8 Gud. lat.): N.29.

368 (11 Gud. gr.): N.33 (2).

368 (Gud. 11): I.86, 87.

369 (Gud. 69): I.92.

369 (69 Gud. gr.): N.33.

369. 370 (Gud. 69): I.93.

370 (69 Gud. gr.): N.34.

Helmstad.

757.837: I.73.

Weissenb.

64: I.76.

Zaragoza, Bibl. d. Cat. d. Pil.

1106 (ol. 562): II.89 (2), 90.

1115 (olim 1427): II.26.

\section{Zeitz, Stiftsbibliothek}

66: I.72.

Zürich, Stadtbibliothek

C 50: II.97.

C 136: I.39, 43; II.34. 



\section{Bibliography}

The bibliography in Diels comprises four lists: (1) the Verzeichnis der ausgenutzten Bibliotheken und Bibliothekskataloge (List of Consulted Libraries and Library Catalogues) included in the front matter (pp. XI-XXIII) of the second issue of the catalogue (1906) and reproduced in the cumulative edition (1906) of the two issues (1905 and 1906) with the same pagination; (2) some additional entries to this list in the front matter of the Nachtrag (Supplement, 1908) (pp. 23-24); and, at the end of the Nachtrag, (3) a Verzeichniss gedruckter Anonymi medici graeci (List of Printed Anonymous Greek Physicians) followed by (4) a Verzeichniss von Nachschlagwerken (Inventory of Reference Works) (pp. 70-71 and 71-72, respectively). All of these lists are reproduced here with some modifications which are detailed in the introductory notes for each of these bibliographies. 



\section{Catalogues of Manuscripts}

The list of libraries and catalogues below merges the two original lists of Diels (that is, the 1906 and 1908 lists). Furthermore, whereas Diels' bibliography mentions the catalogue(s) of manuscripts in all the languages considered in the lists (Greek, Latin, Arabic, Syriac, and Hebrew), the present bibliography includes only the catalogues of Greek manuscripts.

The format of the resulting list has been slightly modified for purposes of clarity. In the original publication, the bibliographical list includes the full reference of the catalogues under the name of the libraries in each city. As a result, Diels' lists of catalogues by cities are rather dense and do not allow for a general perception of the vast range of works that were consulted to compile the catalogues. The bibliography below proceeds with two separate lists: the first, which lists the catalogues by country, city and library, provides references in an abbreviated form (this is the Short-reference List) and the second list (the Full-reference List) provides the full references of all catalogues cited in the first list.

The Short-reference List proceeds by country identified by its German name, as in Diels. Countries include polities that no longer exist (e.g., Österreich-Ungarn, the Austro-Hungarian Empire) and a grouping utilized by Diels (Türkey und Orient [Turkey and the East], which corresponds approximately to the Ottoman Empire in the early $20^{\text {th }}$ century). The designations of the countries are those of that time (e.g., Russland [Russia]) as is also their extension (portions of modern Greece and the Greek islands do not appear under Griechenland [Greece], for example, but under Türkey und Orient [Turkey and the East], and such a city as Wroclaw, identified here by its German name Breslau, is included in Deutschland (Germany) whereas it is in modernday Poland). Countries, polities, and groupings identified as above are listed here in the alphabetical order of their name whereas in Diels they are listed according to a clockwise movement that proceeds in three concentric circles. The first such circle, which might be identified as Mediterranean and northwestern European, goes from Portugal to Italy passing through France and including Belgium, Holland and Switzerland. The second circle, which might be defined as north-central European, goes from England to Germany to Austria-Hungary. The third circle, ScandinavoAsian in nature, includes Denmak, Sweden and Russia. This repartition is completed by the region on the East, with Greece, Turkey and the East.

In both Diels and the present list, the section devoted to each country lists the cities alphabetically by name. These names are mostly in German (e.g., Athen, Brüssel, 
Florenz, Kopenhagen, Moskau, Venedig), but sometimes in their original form (e.g.,

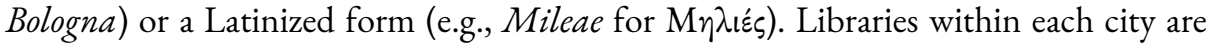
designated, both in Diels and the present bibliography, by the German translation of their name (in most cases) or their original name (often in an abbreviated form and, for some of them, in the original Greek alphabet). All city and library names are printed in boldface for purposes of clarity, both in Diels and here.

The short references of catalogues provided in the Short-reference List here provide a standardized abbreviated form of the catalogue titles. This abbreviation is made of the author's last name or the title of a work, followed by the year of publication. Titles of works always reflect Diels' usage and might be a title sensu proprio, the general title of a series followed by a volume number in such series, or even the title of a journal with the number of the pages where the publication referred to appears.

The Short-reference List is followed here by the Full-reference List, which reproduces the abbreviated form of the title used in the Short-reference List in boldface, immediately followed by the full form of the title according to standard bibliographical practice. Besides providing the key to abbreviations used in the list of catalogues by city and library, this second list allows for a clear vision of the range of catalogues consulted by Diels and his collaborators, something that the original publication does not make possible.

The section devoted to each country opens with the general catalogues as in Diels' original publication. Within each subsection (city and library) items are listed in chronological order of publication. 


\section{Short-reference List}

\section{Allgemeines.}

Haenel 1830; Weinberger 1902.

Serapeum I (1840) - XXXI (1870); Revue des bibliotbèques I (1891) - XIV (1904); Centralblatt für Bibliothekswesen I (1884) - XXI (1904).

\section{BELGIEN.}

Omont 1884-1885 (= Omont, Rev. instr. publ. 1884-1885).

Antwerpen, Stadtbibliothek: Omont 1884-1885: 41-42. Brüssel, Königl. Bibl.: Omont 1884-1885: 1-41; Van den Gheyn 1901-1904;

\section{DÄNEMARK.}

Kopenhagen, Königl. Bibliothek: Graux 1880: 141-238; Universitätsbibl.: Graux 1880: 239-242.

\section{DEUTSCHLAND.}

Blau 1886.

Berlin, Königl. Bibl.: Studemund-Cohn 1890; De Boor 1897. Breslau, Bibl. Korniana: Henschel 1847. Bibl. der Maria-Magdalena-Kirche (jetzt Stadtbibliothek): Henschel 1847. Stadtbibliothek: Krantz 1699; Wolf, 1764; Wachler 1828; Neigebauer 1865; Catalogus Vratislaviensis 1899. Universitätsbibliothek: Schneider 1840/41; Henschel 1847; Neigebauer 1864: 46-48. Dresden, Königl. Bibl.: Schnorr v. Carolsfeld 1882-1883. Erlangen, Universitätsbibliothek: Irmischer 1852. Göttingen, Univ.-Bibl.: Meyer, 1893-1894. Hamburg, Stadtbibliothek: Bibliothecae Uffenbachianae III 1730; Catalogus Bibliothecae Loescheri 1751; Omont 1890: 355-368. Philologica Hamburgensia 1905. Heidelberg, Univ.-Bibl.: Wilken 1817. Jena, Universitätsbibl.: Mylius 1746; Zentralbl. f. Bibl. Wesen 19 1902. Karlsruhe, Großherzogl. Hof- u. Landesbibliothek: Die Hdss. der großherzogl.-badischen Hof-u. Landesbibliothek 1891-1901. Leipzig, Ratsbibliothek: Naumann 1838. Universitätsbibliothek: Feller 1686; Gardthausen 1898. München, Hof- und Staatsbibl.: Hardt 1806-1812. Tübingen, Univ.-Bibl.: Keller 1840; 1841; Klüpfel 1843; Schmid 1902, 1903; Nestle 1903. Wolfenbüttel, Herzogl. Bibl.: Ebert 1827; v. Heinemann 1884-1888,1890-1903,1903. Zeitz, Stiftsbibliothek: Müller 1806-1818; Wegener 1876; Bech 1881. 


\section{ENGLAND.}

Daremberg 1853.

Cambridge, Gonville and Caius: Smith 1849. Peterhouse: James 1899. St Johns' College: Cowie 1842-1843. Trinity College: Sinker 1891; James 19001904. Universitätsbibliothek: Daremberg 1853: 164-166; Halliwell 18561867. Cheltenham: Catalogus libr. mss. in bibl. Th. Phill. 1837; Daremberg 1853: 119-156. Dublin, Trinity College: Schenkl 1896: 43-69; Abbott 1900. Holkham, Bibl. des Gr. Leicester: Roscoe 1815-1828; Foerster 1884. London, British Museum: Thompson 1881-1884; Arundeliani: (Forshall) 1834; Burneiani: (Forshall) 1840; Harleiani: (Nares) 1808-1812; Regii: Casley 1734; Index 1829. Sloane Mss.: Ayscough 1782; Scott 1904; Stowe Mss.: Cat. of the Stowe Mss. 1895-1896. Additions: Index to the Additions 1783-1835 1849; List of additions 1836-1897: List of Additions 1836-1840 1843; Catalogue of Additions 1841-1845 1850; Catalogue of Additions 1846-1847 1864; Catalogue of Additions 1848-1853 1868; Catalogue of Additions 1854-1860 1875; Catalogue of Additions 1854-1875 1877; Index of the Catalogue of Additions 1854-1875 1880; Catalogue of Additions 1876-1881 1882; Catalogue of Additions 18821887 1889; Catalogue of Additions 1888-1893 1894; Catalogue of Additions 1894-1899 1901. Papyri: Kenyon 1893-1898. Lambeth Palace: Todd 1812. Medical Society: Daremberg 1853: 158-164. Oxford, Bibl. Bodleiana: Codd. mss. ... d'Orvilliani 1806; Black 1845; Coxe 1853; Madan 1895; Daremberg 1853 : 14-62, 100-118, 172. Bibl. aedis Christi: Kitchin 1867. Colleg. Mertonense: Coxe 1852: Part 3. Colleg. Novum: Coxe 1852: Part 7.

\section{FRANKREICH.}

Catalogue général des mss. des bibl. publiques des départements, 7 Bde. 1849-1885; Catalogue général des mss. des bibl. publiques de France. Départements, 43 Bde. 1886-1904; Omont, Départements 1886.

Besançon: Catal. gén. de Fr. Dép. 321897 \& 33 1900. Carpentras: Catal. gén. de Fr. Dép. 34 1901; 35 1899; 361902 \& 1903. Lyon, Bibl. de la Ville: Catal. gén. de Fr. Dép. 301899 \& 1900; 42, 1904. Montpellier, Bibliothèque de l'école de médecine: Catal. gén. des Dép. 1 1849. Orléans: Catal. gén. de Fr. Dép. 12 1889; 42 1904: 563-649. Paris, Bibl. Nationale. [Mellot], Catalogus codd. mss. bibl. regiae 1739-1744; Omont 1886-1888, 1898. Neue Erwerbungen: 1888-1892: Omont 1892; 1892-1893: Omont 1894; 1894-1895: Omont 1896; Omont, Catal. des mss. ... Miller 1897; 1898-1899: Omont 1900; Omont 1903. Kleinere Bibliotheken: Omont 1883. Bibl. Mazarine: Catal. gén. de Fr. 1885-1895. Bibliothèque de la faculté de médecine: Franklin 1864; Omont 1883: 123124. Universitäts-Bibliothek: Chatelain 1892. Reims: Catal. gén. de Fr. Dép. 39 
1904 \& 1906. Rosanbo: Omont, Départements 1886: 67-72. Sens: Catal. gén. de Fr. Dép. 6 1887: 147-204.

\section{GRIECHENLAND.}

Pervanoglu 1868.

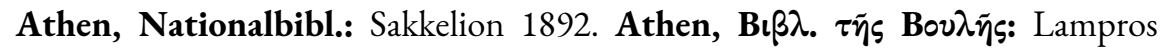
1905-1907. Mileae (Griechenland): Papadopulos-Kerameus 1901.

\section{HOLLAND.}

Omont, Pays-Bas 1886.

Amsterdam, Universitäts-Bibliothek: Omont, Pays-Bas 1886: 195-198; Mendes da Costa 1902. Leiden, Universitäts-Bibliothek: Catalogus librorum ... bibl. publ. universitatis Lugduno-Batav. 1716; Geel 1852.

\section{ITALIEN.}

Tomasini 1639, 1650; Blume 1824-1836, 1834; Vogel 1841-1842; Statistica del regno d'Italia 1865; Mazzatinti 1887, 1891-1903; Allen 1890; Ehrhard 1893; Statistica delle biblioteche 1893-1894; Martini 1893, 1896, 1902; Bassi 1897-1901; Biblioteche governative 1900 .

Bologna: Olivieri, Festa 1895; Puntoni 1896/2; Bibl. Comunale: Olivieri, Festa 1895: 467-481. Bibl. Universitaria: Olivieri, Festa 1895: 387-466; Puntoni 1896/2; Biblioteche governative 1900: 275. Florenz, Bibl. Mediceo Laurentiana: Bandini 1764-70; Rostagno-Festa 1893; Rostagno 1894, 1898. Kleinere Bibliotheken: Vitelli 1894. Bibl. Marucelliana: Biagi 1888; Vitelli 1894: 558562;. Bibl. Nazionale (= Magliabecchiana): Vitelli 1894: 543-557. Bibl. Riccardiana: Vitelli 1894: 473-542. Genua: Ehrhardt 1893. Bibl. della Congregazione delle Missioni Urbane (mit Bibl. d. Fil. Sauli): Ehrhardt 1893: 192-218. Grottaferrata, Klosterbibliothek: Rocchi 1883. Mailand, Archivio del Capitolo metropolitano: Martini 1893: 39-46. Mailand, Bibliotheca Ambrosiana: Martini et Bassi 1906. Bibl. Trivulziana: Porro 1884; Martini 1893: 373-385. Messina, Universität: Blume 1834: 228; Rühl 1889; Fraccaroli 1897; Biblioteche governative 1900: 309-323. Modena, Bibl. Estense: Puntoni 1896/1. Neapel, Biblioteca Borbonica: Cyrillus 1826-1832. Bibliot. dei Gerolamini: Martini 1896: 387-415. Bibl. di S. Giovanni di Carbonara: Montfaucon 1739: 1.231-233; Fabricius 1796; Capasso 1878: 586-590. Bibl. Oratoriana: Mandarini 1897. Padua: Tomasini 1639. S. Antonius: Minciotti 1842; Iosa 1886. Bibl. universitaria: Landi 1902; Biblioteche governative 1900: 345-353. Palermo, Jesuitenkolleg (jetzt Bibl. nazionale): Blume 1834: 230- 
231. Biblioteca nazionale (mit Bibl. des Jesuitenkollegs): Martini 1893: 47138; Biblioteche governative 1900: 89-97. Parma, Bibl. Palatina: Blume 1834: 234-236; Martini 1893: 149-205; Biblioteche governative 1900: 217-223; Modona 1896. Pistoja, Biblioteca Fabroniana: Festa 1897: 226-228; Mazzatinti I 1890: 268-277. Bibl. Forteguerri: Mazzatinti I 1890: 239-267; Festa 1897: 225. Ravenna, Biblioteca Classense: Martin, Mélanges Graux 1884; Mazzatinti IV 1894 144-254; V 1895 3-47; Rom, Bibl. Angelica: Franchi de' CavalieriMuccio 1896; Piccolomini 1898. Bibl. Barberina: Blume 1834: 148-155. Bibl. Casanatensis: Allen 1890: 45-48; Bancalari 1894. Bibl. Corsiniana (= Acc. d. Lincei): Blume 1834: 188-191; Pélissier 1889, 1891; Pierleoni 1901; Gabrieli 1905. Bibl. St. Peter (Basilicana): Montfaucon 1739: 1.156-159; Allen 1890: 56; Heiberg 1896. Bibl. Vallicellana: Martini 1902. Bibl. Vaticana: Palatini graeci: Stevenson 1885; Reg. Sueciae et Pii II: Stevenson 1888; Ottoboniani: Feron et Battaglini. 1893; Urbinates: Stornajolo 1895. Turin, Bibl. Nazionale (Universitaria): Pasinus 1749; Peyron, 1820; Zuretti 1896; (Cipolla) 1904. Venedig, Bibl. Nazionale Marciana: [Zanetti] 1740; Castellani 1895. Kleinere Bibliotheken: Tomasini 1650. Bibl. monasterii St. Michaelis: Mittarelli 1779. Verona: Blume 1824-1836: 1.259-271; Omont 1891. La capitolare biblioteca: Giuliari 1875-1876; Omont 1891: 490-495.

\section{ÖSTERREICH-UNGARN.}

Goldmann 1881; Huemer 1885, 1887.

Böhmen: Balbini 1780: 1-47. Budapest, Universitätsbibl.: Szilágyi 1881-1894. Raudnitz (Böhmen): Bibl. d. Fürsten Lobkowitz: Passow 1825: VI. adn.; Dworzak 1843. Sáros-Patak, Bibl. des reform. Collegiums: Haenel 1840. Wien: Jesuitenkollegium: Gollob 1908.

\section{PORTUGAL.}

Graux et Martin 1892: 299-308.

\section{RUSSLAND.}

Thraemer 1892; Vater 1843.

Moskau: v. Gebhardt 1898. Synodalbibl.: Matthaei 1805. St. Petersburg: Kaiserl. öffentl. Bibl.: Muralt 1864. Warschau, Zamoyskische Majoratsbiblithek: Foerster 1898, 1900. 


\section{SCHWEDEN.}

Graux et Martin 1889.

Upsala, Universitätsbibl.: Sparfwenfeldt 1706; Svedelius 1806; Graux et Martin 1889: 314-358.

\section{SCHWEIZ.}

Omont, Suisse 1886; Omont, Supplément Suisse 1891; Meier 1887.

Basel, Universitäts-Bibliothek: Montfaucon 1739: 607-615; Haenel 1830: 511-660; Omont, Suisse 1886: 386-419; Omont, Supplément Suisse 1891:22-24. Bern, Stadtbibliothek: Hagen 1875. Genf, Stadtbibliothek: Senebier 1779; Omont, Suisse 1886: 430-440. Zürich, Stadtbibliothek: Weidmann 1841; Omont, Suisse 1886: 441-445.

\section{SPANIEN.}

Heine, Serapeum 7 1846; Heine, Serapeum 8 1847; Volger 1858, 1859; Ruelle 1875; Graux 1879; Graux et Martin 1892; Beer 1893

Escurial: Miller 1848. Madrid, Privatbibliothek des Königs: Graux et Martin 1892: 55-125. Nationalbibliothek: Iriarte 1769; Miller 1886; Omont 1897. Salamanca, Universitäts-Bibliothek: Volger, Philologus 14 1859; Graux et Martin 1892: 145. Toledo, Archivo historico = Bibl. Capitolare: Haenel 1830: 983-999; Ruelle 1875: 506-508; Graux et Martin 1892: 32, 229, 293. Bibl. del cabillo de la iglesia catedral: Graux et Martin 1892: 293. Zaragoza, Bibl. d. Pilar: Graux et Martin 1892: 207.

\section{TÜRKEI UND ORIENT.}

Coxe 1858; Papadopulos-Kerameus 1887.

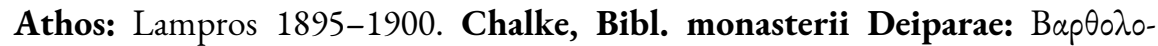

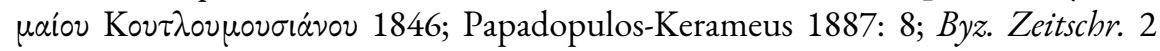
1893; Papadopulos-Kerameus 1900; Boyens, 1901; Byz. Zeitschr. 10 1901;. Constantinopel: Weißenborn 1857; Pbilolog. 5 1850: 758-762; Foerster 1877; Codera 1891;. Bibl. des Sultans: Mordtmann 1854; Blaß 1888; Foerster, Philolog. 42 1884: 167. Gallipoli, Bibl. d. Kirche d. H. Nikolaus: PapadopulosKerameus 1887: 6-12. Jerusalem: Ehrhard 1891-1892; Papadopulos-Kerameus,

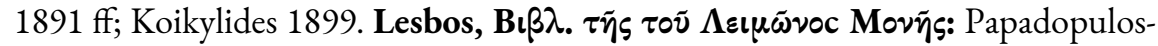
Kerameus, 1886. (Mitylene), Bibl. d. Gymnasiums: Papadopulos-Kerameus 1886: 132; 1888: 143. Patmos: Guérin 1856: 101-120; Migne 1865; Bidez et Parmentier 1896. Bibl. des Johannis-Klosters: Guérin 1856: 101-119. 
clxiv

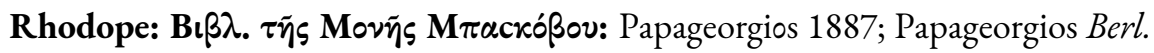

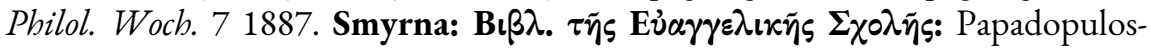
Kerameus, 1877. The Greek library at Smyrna: The Academy 1880. 


\section{Full-reference List}

Centralblatt für Bibliothekswesen 1 (1884)-21 (1904).

Revue des bibliothèques 1 (1891)-14 (1904).

Serapeum 1 (1840)-31 (1870).

Abbott 1900: Thomas Kingsmill Abbott, Catalogue of the manuscripts in the Library of Trinity College, Dublin. Dublin: Hodges, Figgis, \& Co., and London: Longman, Green, \& Co., 1900.

Allen 1890: Thomas William Allen, Notes on Greek Manuscripts in Italian Libraries. London: David Nutt, 1890.

Ayscough 1782: Samuel Ayscough, $A$ Catalogue of the Manuscripts preserved in the British Museum hitherto undescribed: consisting of Five Thousand Volumes; includeing the Collections of Sir Hans Sloane, Bart, the Rev. Thomas Birch, D,D, and about Five Hundred Volumes bequeathed, presented, or purchased at various Times. Volume II containing Medicine, Natural History, Voyages, Grammars, E'cc, Literary History, Cat. of the mss. pres. in the Brit. Mus. London 1782, Biography, Letters, Poetry, Judicial Astrology, Magic, Miscellaneous, MSS. in Icelandic, and Oriental Languages, with Two Indices. London: The Author, 1782.

Balbini 1780: Bohuslai Balbini, Bohemia docta, Opus posthumus editum, notisque illustratum ab Raphaele Ungar, Pars 3. Pragae: Characteribus Haeredum Rosenmüller per Mathiam Glatz Factorem, 1780.

Bancalari 1894: Francesco Bancalari, "Index codicum graecorum Bibliothecae Casanatensis”, Studi italiani di filologia classica 2 (1894), pp. 161-207.

Bandini 1764-1770: Angelo Maria Bandini, Catalogus codicum manuscriptorum Bibliothecae Mediceae Laurentianae ..., Tomus I-III. Florentiae: Typis Caesareis (Tomus I); Typis Regiis (Tomi II-III), 1764-1770.

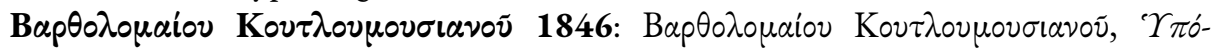

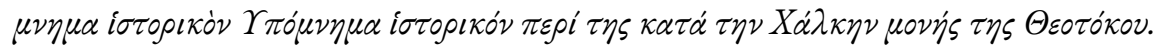

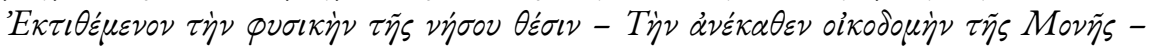

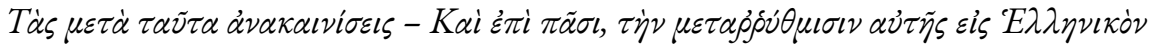

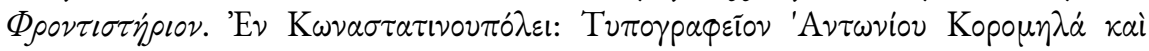
$\Pi \lambda \alpha \dot{\tau} \omega \nu \circ \varsigma \Pi \alpha \sigma \pi \alpha \lambda \lambda \dot{\eta}, 1846$.

Bassi 1897-1901: Domenico Bassi, "Notizie di codici greci nelle biblioteche italiane", Rivista di filologia e d'istruzione classica 25 (1897), pp. 265-276; 26 (1898), pp. 118-124; 29 (1901), pp. 77-83.

Bech 1881: Fedor Bech, Verzeichniss der alten Handschriften und Drucke in der Domberren-Bibliothek zu Zeitz aufgestellt und mit einem Vorworte zur Geschichte der Bibliothek. Berlin: Weidmannsche Buchhandlung, 1881.

Beer 1893: Rudolf Beer, Handschriftenschätze Spaniens. Bericht über eine im Auftrage der kaiserlichen Akademie der Wissenschaften in den Jabren 1886-1888 
durchgefübrte Forschungsreise (Sitzungsberichte der philosophisch-historischen Classe der kaiserlichen Akademie der Wissenschaften 128, VIII. Abhandlung; 129, VI. Abhandlung). Wien: Kaiserliche Akademie der Wissenschaften, 1893.

Biagi 1888: Guido Biagi, Indice del Mare Magnum di Francesco Marucelli (Ministero della Pubblica Istruzione, Indici e cataloghi 9). Roma: [Ministero della Pubblica Istruzione], 1888.

Biblioteche governative 1900: Ministero della Pubblica Istruzione, Le Biblioteche Governative Italiane nel MDCCCXCVIII. Notizie storiche, bibliografiche e statistiche. Roma: Società Editrice Dante Alighieri, 1900.

Bibliothecae uffenbachianae III 1730: Bibliothecae Uffenbachianeae Universalis Tomus III. Exhibens integrum manuscriptorum tam veterum quam recentium adparatum, quem Z. C. Uffenbach summo studio atque labore conlegit, nunc vero bibliophilis aequo pretio offert. Francofurti ad Moenum: Jo. Benj. Andreae \& Henr. Hort, 1730.

Bidez et Parmentier 1896: Joseph Bidez and Léon Parmentier, "Notes sur quelques manuscrits de Patmos”, Revue de philologie 20 (1896), pp. 38-40, 116-125.

Black 1845: William Henry Black, Descriptive, Analytical, and Critical Catalogue of the Manuscripts Bequeathed to the University of Oxford by Elias Ashmole ..., also of Some Additional MSS. Contributed by Kingsley, Lbuyd, Borlase and Others. Oxford: University Press, 1845.

Blaß 1888: Friedrich Wilhelm Blaß, "Die griechischen und lateinischen Handschriften im Alten Serail zur Konstantinopel”; "Nachtrag”, Hermes. Zeitschrift für classische Philologie 23 (1888), pp. 219-233, 622-625.

Blau 1886: August Blau, "Verzeichniss der Handschriftenkataloge der deutschen Bibliotheken”, Centralblatt für Bibliothekswesen 3 (1886), pp. 1-35, 49-108, 160.

Blume 1824-1836: Friedrich Blume, Iter italicum, Erster Band. Archive, Bibliotheken und Inschriften in den Sardinischen und OEsterreichischen Provinzen; Zweiter Band. Archive, Bibliotheken und Inschriften in Parma, Modena, Massa, Lucca, Toscana, dem Kirchenstaat und S. Marino; Dritter Band. Archive, Bibliotheken und Inschriften in der Stadt Rom; Vierter und Lezter Band. Königreich Neapel, nebst Nachträgen und Registern zu allen vier Bänden, und zur Bibliotheca Librorum MSS. Italica. Berlin und Stetting: In der Nicolaischen Buchhandlung (Bd. 1-2); Halle: Eduard Anton (Bd. 3-4), 1824-1836.

Blume 1834: Friedrich Blume, Bibliotheca Librorum Manuscriptorum Italica. Indices bibliothecarum Italiae ex schedis Maieri eslingensis, Haenelii lipsiensis, Gottlingii ienensis, Car. Wittii, suisque propriis. In supplementum itineris italici. Gottingae: impensis Bibliopolii Dietericiani, 1834.

Boyens 1901: Joseph Boyens, "Catalogus codicum hagiographicorum graecorum bibliothecae monasterii Deiparae in Chalce insula”, Analecta Bollandiana 20 (1901), pp. 45-70. 
Byz. Zeitschr. 2 1893: Athanasios Papadopoulos Kerameus, "Handschriftliches zu Ignatios Diakonos”, Byzantinische Zeitschrift 2 (1893), pp. 126-131.

Byzant. Zeitschr. 10 1901: Eduard Kurz “A. Papadopoulos-Kerameus, 'H દ̇v $\tau \tilde{\omega}$

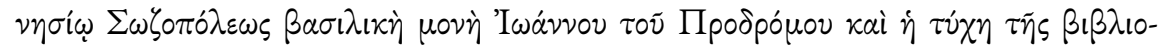

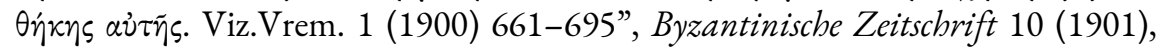
p. 667.

Capasso 1878: Bartolomeo Capasso, "Sulla spogliazione delle biblioteche napolitane nel 1718. Notizie e Documenti.”, Archivio storico per le provincie Napoletane 3 (1878), pp. 563-594.

Casley 1734: David Casley, A Catalogue of the Manuscripts of the Kings'Library: An Appendix to the Catalogue of the Cottonian Library Together with an Account of Books burnt or damaged by a late Fire. One Hundred and Fiftly Specimens of the Manner of Writing in different Ages, from the Third to the Fifteenth Century, in Copper-Plates: and some Observations upon MSS, in a Preface. London: the Author, 1734.

Castellani 1895: Carolus Castellani, Catalogus codicum graecorum qui in bibliothecam D. Marci Venetiarum inde ab anno MDCCXL ad haec usque tempora inlati sunt. Venetiis: sumptibus Ferd. Ongania et F. M. Visentini fratrum, 1895.

Cat. of the Stowe Mss. 1895-1896: Catalogue of the Stowe Manuscripts in the British Museum, volume 1: Text; volume 2: Index. London: British Museum, 1895-1896.

Catal. gén. de Fr. 1885-1895: Auguste Molinier, Catalogue des manuscrits de la Bibliothèque Mazarine (Catalogue général des manuscrits des bibliothèques publiques de France), 4 volumes. Paris: Librairie Plon, E. Plon, Nourrit et $\mathrm{C}^{\text {ie, }}$ 1885-1895.

Catal. gén. de Fr. Dép. 6 1887: Auguste Molinier, Catalogue général des manuscrits des bibliothèques publiques de France, Départements, Tome VI: Auxerre ... Sens ... Paris: Librairie Plon, E. Plon, Nourrit et C $\mathrm{C}^{\mathrm{ie}}, 1887$.

Catal. gén. de Fr. Dép. 12 1889: Charles Cuissard, Catalogue général des manuscrits des bibliotbèques publiques de France, Départements, Tome XII: Orléans. Paris: Librairie Plon, E. Plon, Nourrit et C $C^{\text {ie }}, 1889$.

Catal. gén. de Fr. Dép. 30 1899: Auguste Molinier et Félix Desvernay, Catalogue général des manuscrits des Bibliothèques publiques de France, Départements, Tome XXX: Lyon. Première partie. Paris: Librairie Plon, E. Plon, Nourrit et C $C^{\text {ie }}, 1899$.

Catal. gén. de Fr. Dép. 30 1900: Auguste Molinier et Félix Desvernay, Catalogue général des manuscrits des Bibliothèques publiques de France, Départements, Tome XXX: Lyon. Seconde partie. Paris: Librairie Plon, E. Plon, Nourrit et C ${ }^{\text {ie }}, 1900$.

Catal. gén. de Fr. Dép. 32 1897: Auguste Castan, Catalogue général des manuscrits des bibliothèques publiques de France, Départements, Tome XXXII: Besançon. Tome I. Paris: Librairie Plon, E. Plon, Nourrit et Ce, 1897. 
Catal. gén. de Fr. Dép. 33 1900: Auguste Castan, Catalogue général des manuscrits des bibliothèques publiques de France, Départements, Tome XXXIII: Besançon, Tome II. Première partie. Paris: Librairie Plon, E. Plon, Nourrit et C $C^{\text {ie }}, 1900$.

Catal. gén. de Fr. Dép. 34 1901: Léopold Duhamel, Catalogue général des manuscrits des bibliothèques publiques de France, Départements, Tome XXXIV: Carpentras, Tome I. Paris: Librairie Plon, Plon-Nourrit et C $C^{\mathrm{ie}}, 1901$.

Catal. gén. de Fr. Dép. 35 1899: Léopold Duhamel et Joseph Liabastres, Catalogue général des manuscrits des bibliothèques publiques de France, Départements, Tome XXXV: Carpentras, Tome II. Paris: Librairie Plon, E. Plon, Nourrit et C ${ }^{\text {ie }}, 1899$.

Catal. gén. de Fr. Dép. 36 1902: Joseph Liabastres, Catalogue général des manuscrits des bibliotbèques publiques de France, Départements, Tome XXXVI: Carpentras, Tome III. Première partie. Paris: Librairie Plon, Plon-Nourrit et C ${ }^{\text {ie, }}$ 1902.

Catal. gén. de Fr. Dép. 36 1903: Léon-Honoré Labande, Catalogue général des manuscrits des bibliothèques publiques de France, Départements, Tome XXXVI: Carpentras, Tome III. Deuxième partie. Paris: Librairie Plon, Plon-Nourrit et $\mathrm{C}^{\text {ie }}$, 1903.

Catal. gén. de Fr. Dép. 39 1904: Henri Loriquet, Catalogue général des manuscrits des Bibliothèques publiques de France, Départements, Tome XXXIX: Reims, Tome II. Première partie. Paris: Librairie Plon, Plon-Nourrit et C ${ }^{\text {ie }}, 1904$.

Catal. gén. de Fr. Dép. 39 1906: Henri Loriquet, Catalogue général des manuscrits des Bibliothèques publiques de France, Départements, Tome XXXIX: Reims, Tome II. Deuxième partie. Paris: Librairie Plon, Plon-Nourrit et $C^{\text {ie }}, 1904$.

Catal. gén. de Fr. Dép. 42 1904: Félix Desvernay, Catalogue général des manuscrits des bibliothèques publiques de France, Départements, Tome XLII, Supplément, Tome III: Lyon, Orléans. Paris: Librairie Plon, Plon-Nourrit et C $C^{\text {ie }}, 1904$.

Catal. gén. des Dép. 1 1849: Guillaume Libri, "Catalogue des manuscrits de la Bibliothèque de l'Ecole de médecine de Montpellier”, in Félix Raviasson et Guillaume Libri, Catalogue général des manuscrits des bibliothèques publiques des départements, Tome I: Autun, Laon, Montpellier, Albi. Paris: Imprimerie nationale, 1849 , pp. 279-477.

Catalogue général des mss. des bibl. publiques de France. Départements. 43 Bde. 1886-1904: Catalogue général des manuscrits des bibliothèques publiques de France, Départements 1-43. Paris: Librarie Plon, E. Plon, Plon-Nourrit et $\mathrm{C}^{\text {ie }}$, 1886-1897 (= 1-32); Librairie Plon, Plon-Nourrit et $C^{\mathrm{ie}}, 1900-1904$ (=33-43).

Catalogue général des mss. des bibl. publiques des départements. 7 Bde. 18491885: Catalogue général des manuscrits des bibliothèques publiques des départements publié sous les auspices du Ministre de l'Instruction publique (tomes 1-2, 4-7) du Ministre d'Etat (tome 3), 7 tomes. Paris: Imprimerie nationale (tomes 1, 4-7); Imprimerie Impériale (tomes 2-3), 1849-1885. 
Catalogue of Additions 1841-1845 1850: Catalogue of Additions to the Manuscripts in the British Museum in the Years 1841-1845. London: British Museum, 1850.

Catalogue of Additions 1846-1847 1864: Catalogue of Additions to the Manuscripts in the British Museum in the Years 1846-1847. London: British Museum, 1864.

Catalogue of Additions 1848-1853 1868: Catalogue of Additions to the Manuscripts in the British Museum in the Years 1848-1853. London: British Museum, 1868.

Catalogue of Additions 1854-1860 1875: Catalogue of Additions to the Manuscripts in the British Museum in the Years 1854-1860. London: British Museum, 1875.

Catalogue of Additions 1854-1875 1877: Catalogue of Additions to the Manuscripts in the British Museum in the Years 1854-1875, volume II. London: British Museum, 1877.

Catalogue of Additions 1876-1881 1882: Catalogue of Additions to the Manuscripts in the British Museum in the Years 1876-1881. London: British Museum, 1882.

Catalogue of Additions 1882-1887 1889: Catalogue of Additions to the Manuscripts in the British Museum in the Years 1882-1887. London: British Museum, 1889.

Catalogue of Additions 1888-1893 1894: Catalogue of Additions to the Manuscripts in the British Museum in the Years 1888-1893. London: British Museum, 1894.

Catalogue of Additions 1894-1899 1901: Catalogue of Additions to the Manuscripts in the British Museum in the Years 1894-1899, Part I: Descriptions; Part II: Index. 2 volumes. London: British Museum, 1901.

Catalogus Bibliothecae Loescheri 1751: Catalogus Bibliothecae viri summi D. Valentini Ernesti Loescheri Theologi Saxoni, cuius pars I continens bibliothecam theologicam hastae publicae subiicietur D. VII. seqq. jan 1750 horis a meridie consuetis, in aedibus viduae B. Hermanni Poligraphi. Dresdae et Lipsiae: apud Harpeterum, 1751.

Catalogus librorum ... bibl. publ. universitatis Lugduno-Batav. 1716: Wolferdius Senguerdius, Jacobus Gronovius, and Johannes Heyman, Catalogus librorum tam impressorum quam manuscriptorum bibliothecae publicae universitatis lugduno-batavae ... Lugduni apud Batavos: Sumptibus Petri van der Aa, 1716.

Catalogus libr. mss in bibl. Th. Phill. 1837: Catalogus librorum manuscriptorum in bibliotheca D. Thomae Phillipps Bart. A.D. 1837. [Middle Hill]: Impressus typis Medio-Montanis, 1837. 
Catalogus Vratislaviensis 1889: Catalogus codicum graecorum qui in Bibliotheca Urbica Vratislaviensi adservantur a philologis vratislaviensibus compositus. Civitatis vratislaviensis sumptibus impressus. Accedit Appendix qua Gymnasii regii Fridericiani codices graeci describuntur. Vratislavae: Apud Guilelmum Koebnerum, 1889.

Chatelain 1892: Emile Chatelain, Catalogue des manuscrits de la bibliothèque de l'université. Paris: Honoré Champion, 1892.

(Cipolla) 1904: Ettore Stampini, Gaetano De Sanctis, Carlo Cipolla, Carlo Frati, "Inventario dei Codici superstiti greci e latini antichi della Biblioteca nazionale di Torino", Rivista di filologia e d'istruzione classica 32 (1904), pp. 385-542.

Codera 1891: Francisco Codera y Zaidin, "Informes. I. Catálogos de bibliotecas de Constantinopla", Boletín de la Real Academia de la Historia 18 (1891), pp. 297303.

Codd. mss. ... D'Orvilliani 1806: [Thomas Gaisford], Codices manuscripti, et impressi cum notis manuscriptis, olim d'Orvilliani, qui in Bibliotheca Bodleiana apud Oxonienses adservantur. Oxonii: E Typographeo Clarendoniano, 1806.

Cowie 1842-1843: Benjamin Morgan Cowie, A descriptive catalogue of the manuscripts and scarce books in the library of St. John's College, 2 volumes (Publications of the Cambridge Antiquarian Society 6). Cambridge: J. \& J. J. Deighton, and T. Stevenson, and London: John W. Parker, 1842-1843.

Coxe 1852: Henry O. Coxe, Catalogus Codicum MSS. qui in Collegiis Aulisque Oxoniensibus hodie adservantur, 2 volumes. Oxonii: E Typographo Academico, 1852.

Coxe 1853: Henry O. Coxe, Catalogi Codicum Manuscriptorum Bibliothecae Bodleianae Pars Prima Recensionem Codicum Graecorum Continens. Oxonii: E Typographo Academico, 1853.

Coxe 1858: Henry O. Coxe, Report to Her Majesty's Government, on the Greek Manuscripts yet Remaining in Libraries of the Levant. London: George E. Eyre and William Spottiswoode, 1858.

Cyrillus 1826-1832: Salvator Cyrillus, Codices Graeci MSS. Regiae Bibliothecae Borbonicae descripti, atque illustrati, Tomus I. Qui complectitur bibliothecam sacram. Tomus II. Neapoli: Ex Regia Typographia, 1826-1832.

Daremberg 1853: Charles Daremberg, Notices et extraits des manuscrits médicaux grecs, latins et français des principales bibliotbèques de l'Europe. $\mathrm{I}^{\mathrm{re}}$ partie. Manuscrits grecs d'Angleterre suivis d'un fragment inédit de Gilles de Corbeil et de scolies inédites sur Hippocrate. Paris: Imprimerie Impériale, 1853.

De Boor 1897: Carl de Boor, Verzeichniss der griechischen Handschriften der Königlichen Bibliothek zu Berlin, II. Berlin: A. Asher \& Co, 1897.

Die Hdss. der großherzogl.-badischen Hof-u. Landesbibliothek in Karlsrube 1891-1901 (actually 1891-1896): Die Handschriften der grossherzoglich-badischen Hof- und Landesbibliothek in Karlsrube. Wilhelm Brambach, I. Geschichte 
und Bestand der Sammlung; Wilhelm Brambach, II. Orientalische Handschriften; Alfred Holder, III. Die durlacher und rastatter Handschriften; Wilhelm Brambach, IV. Die karlsruber Handschriften. Karlsruhe: Ch. Th. Gross, 1891-1896.

Dworzak 1843: Dworzak, "Merkwürdigkeiten der Fürstlich von Lobkowitzischen Schlossbibliothek zu Raudnitz an der Elbe in Böhmen", Serapeum - Zeitschrift für Bibliothekwissenschaft, Handschriftenkunde und ältere Litteratur 4 (1843) p. 1-11.

Ebert 1827: Friedrich Adolf Ebert, Bibliothecae Guelferbytanae codices graeci et latini classici. Lipsiae: apud Steinackerum et Harknochium, 1827.

Ehrhard 1891-1892: Albert Ehrhard, "Die griechische Patriarchal-Bibliothek von Jerusalem. Ein Beitrag zur griechischen Palaeographie”, Römische Quartalschrift für christliche Altertbumskunde und für Kirchengeschichte 5 (1891), pp. 217-265, 329-331, 383-384; 6 (1892), pp. 339-365.

Ehrhard 1893: Albert Ehrhard, "Zur Catalogisirung der kleineren Bestände griechischer Handschriften in Italien (I. Genova]", Zentralblatt für Bibliothekswesen 10 (1893), pp. 189-218.

Fabricius 1796: Gaetano d'Ancora, "Codices graeci MSS. qui adservantur Neapoli in Bibliotheca Augustinensium S. Iohannis ad Carbonariam”, in Jo. Alberti Fabricii, Bibliotheca graeca sive notitia scriptorum veterum graecorum quorumcumque monumenta integra aut fragmenta edita exstant tum plerorumque e mss. ac deperditis ab auctore recognita. Editio tertia variorum curis emendatior atque auctor curante Gottlieb Christophoro Harles. Accedunt Christophori Augusti Heumanni Supplementa inedita, Volumen quintum. Hamburgi: apud Carolum Ernestum Bohn, Lipsiae: Ex Officina Breitkopfia et Haertelia, 1796, pp. 796-800.

Feller 1686: L. Joachim Feller, Catalogus codicum manuscriptorum Bibliothecae Paulinae in Academia Lipsiensi. Lipsiae: Joh. Frid. Gleditsch, 1686.

Feron et Battaglini 1893: Ernest Feron et Fabio Battaglini, Codices manuscripti graeci Ottoboniani Bibliothecae Vaticanae descripti. Romae: Ex Typographeo Vaticano, 1893.

Festa 1897: Nicola Festa, "Indice de' codici greci di Lucca e di Pistoia", Studi italiani di filologia classica 5 (1897), pp. 221-230.

Foerster 1877: Richard Foerster, De antiquitatibus et libris manuscriptis constantinopolitanis commentatio. Rostochii: Formis Academicis Adlerianis, 1877.

Foerster 1884: Richard Foerster, "Mittheilungen aus handschriften. 1. Handschriften in Holkham", Philologus. Zeitschrift fur das klassische Alterthum 42 (1884), pp. 158-167.

Foerster 1898: Richard Foerster, "Zur Handschriftenkunde und Geschichte der Philologie. V. Eine griechische Handschrift in Russisch-Polen und das Anthologion des Orion", Rheinisches Museum für Philologie Neue Folge 53 (1898), pp. 547-574. 
Foerster 1900: Richard Foerster, "Zur Handschriftenkunde und Geschichte der Philologie. VI. Handschriften der Zamoyski'schen Bibliothek”, Rheinisches Museum für Pbilologie Neue Folge 55 (1900), pp. 435-459.

Foerster Pbilolog. 421884 167: Richard Foerster, "Eine handschrift des Serail", Philologus. Zeitschrift fur das klassische Alterthum 42 (1884), p. 167.

(Forshall) 1834: [Josiah Forshall], Catalogue of the Manuscripts in the British Museum, New Series, Volume I, Part I. The Arundel Manuscripts. London: British Museum, 1834.

(Forshall) 1840: [Josiah Forshall], Catalogue of the Manuscripts in the British Museum, New Series, Volume I, Part II. The Burney Manuscripts. London: British Museum, 1840.

Fraccaroli 1897: Giuseppe Fraccaroli, "Dei codici greci del Monastero del SS. Salvatore che si conservano nella biblioteca universitaria di Messina”, Studi italiani di filologia classica 5 (1897), pp. 329-336, 487-514.

Franchi de' Cavalieri-Muccio 1896: Pio Franchi de' Cavalieri e Giorgio Muccio, "Index codicum graecorum Bibliothecae Angelicae", Studi italiani di filologia classica 4 (1896), pp. 33-184.

Franklin 1864: Alfred Franklin, Recherches sur la bibliothèque de la Faculté de médecine de Paris d'après des documents entièrement inédits suivies d'une notice sur les manuscrits qui y sont conservés. Paris, 1864: Auguste Aubry.

Gabrieli 1905: Giuseppe Gabrieli, "Il «Zad al musafir» di ibn Al Gazzar in un ms.

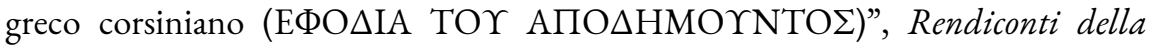
Reale Accademia dei Lincei, Classe di scienze morali, storiche e filologiche, Serie Quinta, 14 (1905), pp. 29-50.

Gard thausen 1898: Viktor Gardthausen, Katalog der griechischen Handschriften der Universitäts-Bibliothek zu Leipzig (Catalogus codicum manuscriptorum Bibliothecae Universitatis Lipsiensis III - Katalog der Handschriften der Universitätsbibliothek zu Leipzig. III Die griechischen Handschriften). Leipzig: O. Harrassowitz, 1898.

Geel 1852: Jacob Geel, Catalogus librorum manuscriptorum qui inde ab anno 1741 bibliothecae Lugduno Batavae accesserunt. Lugduni Batavorum: E.J. Brill, 1852.

Giuliari 1875-1876: G. B. Carlo Giuliari, "La capitolare biblioteca di Verona", Archivio Veneto 10 (1875) pp. 239-271; 11 (1876) pp. 51-74; 12 (1876) pp. 5679, 274-293.

Goldmann 1881: Arthur Goldmann, "Verzeichniss der österreich-ungarischen Handschriftenkataloge", Centralblatt für Bibliothekswesen 5 (1881), pp. 1-37, 55-73.

Gollob 1908: Eduard Gollob, Medizinische griechische Handschriften des Jesuitenkollegiums in Wien (XIII. Lainz) (Sitzungsberichte der Philosophischhistorischen Klasse der Kaiserlichen Akademie der Wissenschaften in Wien, 158. Band, 5. Abhandlung). Wien: Alfred Hölder, 1908. 
Graux 1879: Charles Graux, "Rapport sur une mission en Espagne”, Archives des Missions scientifiques et littéraires, choix de rapports et instruction. Choix de rapports et instructions publié sous les auspices du Ministère de l'instruction publique et des beaux-arts, $3^{\mathrm{e}}$ série, tome 5 (1879), pp. 111-136.

Graux 1880: Charles Graux. "Notices sommaires des manuscrits de la Grande Bibliothèque Royale de Copenhague", Archives des missions scientifiques et littéraires. Choix de rapports et instructions publié sous les auspices du Ministère de l'instruction publique et des beaux-arts, $3^{\mathrm{e}}$ série, tome 6 (1880), pp. 133-242.

Graux et Martin 1889: Charles Graux, "Notices sommaires des manuscrits grecs de Suède mises en ordre et complétées par Albert Martin”, Archives des Missions scientifiques et littéraires, choix de rapports et instructions. Choix de rapports et instructions publié sous les auspices du Ministère de l'instruction publique et des beaux-arts, $3^{\text {e }}$ série, tome 15 (1889), pp. 293-370.

Graux et Martin 1892: Charles Graux and Albert Martin (ed.), "Rapport sur une mission en Espagne et en Portugal. Notices sommaires des manuscrits grecs d'Espagne et de Portugal", Nouvelles Archives des Missions scientifiques et littéraires. Choix de rapports et instructions publié sous les auspices du Ministère de l'instruction publique et des beaux-arts 2 (1892), pp. 1-322.

Guérin 1856: Victor Guérin, Description de l'île de Patmos et de l'île de Samos. Paris: Auguste Durand, 1856.

Haenel 1830: Gustav Haenel, Catalogi librorum manuscriptorum, qui in bibliothecis Galliae, Helvetiae, Belgii, Britanniae M., Hispaniae, Lusitaniae asservantur, nunc primum editi. Lipsiae: I. C. Hinrichs, 1830.

Haenel 1840: Gustav Haenel, "Ungedruckte Handschriften-Kataloge, II. Manuscripta B. Collegii Ref. S. Patak”, Neue Jabrbücher für Philologie und Paedagogik oder Kritische Bibliothek für das Schubl-und Unterrichtswesen 6. Supplementband (1840), pp. 423-424.

Hagen 1875: Hermannus Hagen, Catalogus codicum bernensium (Bibliotheca Bongarsiana). Bernae: B. F. Haller, 1875 (first published as Pars prior in 1874).

Halliwell 1856-1867: Charles Hardwick and Henry Richards Luard (eds), $A$ Catalogue of the Manuscripts preserved in the Library of the University of Cambridge, 5 volumes. Cambridge: University Press, 1856-1867.

Hardt 1806-1812: Ignaz Hardt, Catalogus Codicum Manuscriptorum Graecorum Bibliothecae Regiae Bavaricae, 5 volumes. Monachii: Typis I. E. Seidelii Solisbacensis, 1806-1812.

Heiberg 1896: Johannes Ludwig Heiberg, "Biblioteksnotizen. IV. Ein Inventar des Archivio di S. Pietro", Philologus. Zeitschrift fur das klassische Alterthum 55 (1896), pp. $742-746$.

Heine, Serapeum 7 1846: Gustav Heine, "Briefliche Mittheilung des Dr. G. Heine aus Berlin an Hofrath Hänel in Leipzig über spanische und portugiesische 
Bibliotheken", Serapeum-Zeitschrift für Bibliotbekwissenschaft, Handschriftenkunde und ältere Litteratur 7 (1846), pp. 193-204.

Heine, Serapeum 8 1847: Gustav Heine, "Zweiter Bericht des Dr. G. Heine in Berlin über seine litterarische Reise in Spanien gerichtet an Hofrath und Prof. Dr. Gustav Hänel und von letzterem mitgetheilt"; "Dritter Bericht des Dr. G. Heine in Berlin über seine litterarische Reise in Spanien, gerichtet an Hofrath und Prof. Dr. Gustav Hänel und von letzterem mitgetheilt”, Serapeum-Zeitschrift für Bibliothekwissenschaft, Handschriftenkunde und ältere Litteratur 8 (1847), pp. 81-95, 103-105.

Henschel 1847: Augustus Guilelmus Eduardus Theodorus Henschel, Catalogus codicum medii aevi medicorum ac physicorum qui manuscripti in bibliothecis vratislaviensibus asservantur. Particula I. Praemissa sunt de bibliothecarum vratislaviensium codicibus manuscriptis medii aevi medicis ac physicis observationes generales. Particula II. Inest synopsis chronologica scriptorum medii aevi medicorum ac physicorum quae codicibus bibliothecarum vratislaviensium continentur. Vratislaviae: Eduardus Trewendt, [1847].

Huemer 1885: Johannes Huemer, "Aus alten Bücherverzeichnissen. (Ein Beitrag zur lateinischen Literaturkunde.)”, Wiener Studien - Zeitschrift für classische Philologie 7 (1885), pp. 325-336.

Huemer 1887: Johannes Huemer, "Iter Austriacum I.”, Wiener Studien - Zeitschrift für classische Philologie 9 (1887), pp. 51-93.

Index 1829: [Frederick Augusta Barnard], Bibliothecae regiae catalogus, Tomus quintus: Londini: Guilelmus Nicol, 1829.

Index of the Catalogue of Additions 1854-1875 1880: Index of the Catalogue of Additions to the Manuscripts in the British Museum in the Years 1854-1875. London: British Museum, 1880.

Index to the Additions 1783-1835 1849: Index to the Additional Manuscripts, with those of the Egerton Collection, preserved in the British Museum, and acquired in the years 1783-1835. London: British Museum, 1849.

Iosa 1886: Antonio Maria Iosa, I codici manoscritti della Biblioteca Antoniana di Padova descritti ed illustrati. Padova: Tipografia del Seminario, 1886.

Iriarte 1769: Joannes Iriarte, Regiae Bibliothecae Matritensis Codices Graeci MSS. Volumen prius. Matriti: E Typografia Antonii Perez de Soto, 1769.

Irmischer 1852: Johann Conrad Irmischer, Handschriften-Katalog der Königlichen Universitätsbibliotbek zu Erlangen. Frankfurt a. M. und Erlangen: der Verfasser, 1852.

James 1899: Montague Rhodes James, A Descriptive Catalogue of the Manuscripts in the Library of Peterhouse, with an Essay on the History of the Library by John Willis Clark. Cambridge: University Press, 1899.

James 1900-1904: Montague Rhodes James, The Western Manuscripts in the Library of Trinity College Cambridge. A Descriptive Catalogue, Volume I containing an 
account of the manuscripts standing in class B; Volume II containing an account of the manuscripts standing in class R; Volume III containing an account of the manuscripts standing in class O; Volume IV containing plates, addenda, corrigenda and index. Cambridge: University Press, 1900-1904.

Keller 1840, 1841: Adalbert Keller, "Die Handschriften der königlichen Universitätsbibliothek in Tübingen", Serapeum-Zeitschrift für Bibliothekwissenschaft, Handschriftenkunde und ältere Litteratur 1 (1840), pp. 124-128, 139-144, 203206; 2 (1841), pp. 358-368.

Kenyon 1893-1898: Frederic G. Kenyon, Greek Papyri in the British Museum. Catalogue with Texts, 2 volumes. London: British Museum, 1893-1898.

Kitchin 1867: George William Kitchin, Catalogus codicum MSS. qui in Bibliotheca Aedis Christi apud Oxonienses adservantur. Oxonii: E Typographo Clarendoniano, 1867.

Klüpfel 1843: Karl Klüpfel, "Die Handschriften der königlichen Universitätsbibliothek in Tübingen”, Serapeum-Zeitschrift für Bibliothekwissenschaft, Handschriftenkunde und ältere Litteratur 4 (1843), pp. 184-190, 205-208.

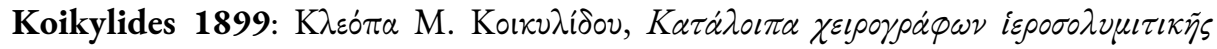

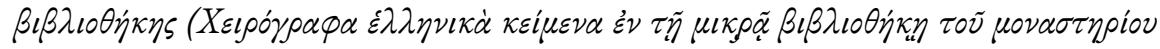

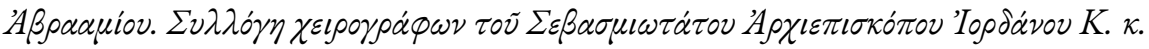

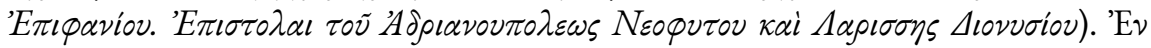

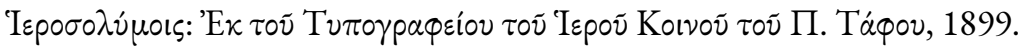

Krantz 1699: Gottlob Krantz, Memorabilia Bibliothecae Publicae Elisabetanae Wratislaviensis, a Fundatore Celeberrimo Rehdigerianae dictae ... Wratislavae: apud Johannem Georgium Steck, 1699.

Lampros 1895-1900: Spyridon P. Lampros, Catalogue of the Greek Manuscripts on Mount Athos, 2 volumes. Cambridge: University Press, 1895-1900.

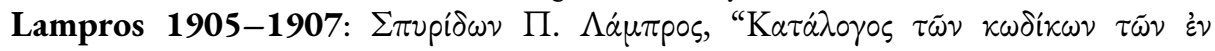

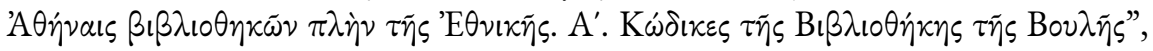

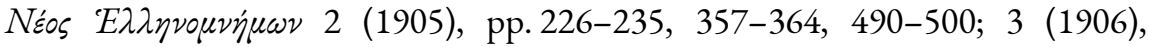
pp. 113-121, 243-248, 447-473; 4 (1907), pp. 105-112, 225-236, 368-374, 476-483.

Landi 1902: Carolus Landi, "Codices graeci bybliothecae Universitatis patavinae", Studi italiani di filologia classica 10 (1902), pp. 18-20, 430-432.

List of Additions 1836-1840 1843: List of Additions to the Manuscripts in the British Museum in the years 1836-1840. London: British Museum, 1843.

Madan 1895: Falconer Madan, A Summary Catalogue of Western Manuscripts in the Bodleian Library at Oxford which have not hitherto been catalogued in the quarto series with references to the Oriental and other manuscripts, volume 3 (Collections received during the 18th Century). Oxford: Clarendon Press, 1895.

Mandarini 1897: Enrico Mandarini, I codici manoscritti della Biblioteca Oratoriana di Napoli illustrati. Napoli and Roma: Andrea e Salvatore Festa, 1897. 
Martin, Mélanges Graux 1884: Albert Martin, "Notice sur les manuscrits grecs de la bibliothèque Classense à Ravenne”, Mélanges Graux. Recueil de travaux d'érudition classique dédié à la mémoire de Charles Graux ... Paris: Ernest Thorin, 1884, pp. 553-556.

Martini 1893: Emidio Martini, Catalogo di manoscritti greci esistenti nelle biblioteche italiane, volume 1, Parte 1. Milano: Ulrico Hoepli, 1893.

Martini 1896: Emidio Martini, Catalogo di manoscritti greci esistenti nelle biblioteche italiane, volume 1, Parte 2. Milano: Ulrico Hoepli, 1896.

Martini 1902: Emidio Martini, Catalogo di manoscritti greci esistenti nelle biblioteche italiane. Volume 2: Catalogus codicum Graecorum qui in Bibliotheca Vallicellana Romae adservantur. Milano: Ulrico Hoepli, 1902.

Martini et Bassi 1906: Aemidius Martini et Dominicus Bassi, Catalogus codicum graecorum Bibliothecae Ambrosianae. Digesserunt -, 2 volumes. Mediolani: Impensis U. Hoepli, 1906.

Matthaei 1805: Christian Friderich de Matthaei, Accurata codicum graecorum MSS. bibliothecarum mosquensium Sanctissimae Synodi notitia et recensio ..., Tomus secundus. Lipsiae: Ex Libraria Joachimica, 1805.

Mazzatinti 1887, 1891-1903: Giuseppe Mazzatinti, Inventari dei manoscritti delle biblioteche d'Italia. Volume I, fascicolo 1: Torino: Ermanno Loescher, 1887; volumi I-XI: Forlì: Luigi Bordandini, 1891-1901; volume XII: Forlì: Tipografia Sociale (Successori Bordandini), 1902-1903.

Mazzatinti I 1890 239-267: Ernesto Gori e Agostino Zanelli, "Pistoia, Biblioteca Forteguerri", in Giuseppe Mazzatinti, Inventari dei manoscritti delle biblioteche d'Italia, 1. Forlì: Luigi Bordandini, 1890, pp. 239-267.

Mazzatinti I 1890 268-277: Agostino Zanelli, "Pistoia, Biblioteca Fabroniana”, in Giuseppe Mazzatinti, Inventari dei manoscritti delle biblioteche d'Italia, 1. Forlì: Luigi Bordandini, 1890, pp. 268-277.

Mazzatinti IV 1894 144-254; V 1895 3-47: Silvio Bernicoli, "Ravenna, Biblioteca Classense", in Giuseppe Mazzatinti, Inventari dei manoscritti delle biblioteche d'Italia, IV, pp. 144-254; V, pp. 3-47. Forlì: Luigi Bordandini, 1894, 1895.

Meier 1887: P. Gabriel Meier, "Verzeichniss der Handschriftenkataloge der schweizerischen Bibliotheken", Centralblatt für Bibliothekswesen 4 (1887), pp. 1-19.

[Mellot], Catalogus codd. mss. bibl. regiae 1739-1744: Catalogus Codicum Manuscriptorum Bibliothecae Regiae. Tomus primus - Tomus quartus. Parisiis: E Typographia Regia, 1739-1744.

Mendes da Costa 1902: Maurits Benjamin Mendes da Costa, Bibliotheek der Universiteit van Amsterdam, Catalogus der Handschriften. II. De Handschriften der Stedelijke Bibliotheke met de latere aanwinsten. Vooral eenige mededeelingen over de geschiedenis van de bibliotheek en hare handschriftenversameling, door den Bibliothecaris Combertus Pieter Burger. Amsterdam: J. H. de Bussy, 1902. 
Meyer 1893-1894: Wilhelm Meyer, Die Handschriften in Göttingen, Bd. 1: Universitätsbibliothek. Philologie, Literärgeschichte, Philosophie, Jurisprudenz; Bd. 2: Universitätsbibliothek. Geschichte, Karten, Naturwissenschaften, Theologie, Handschriften aus Lüneburg; Bd. 3: Universitätsbibliothek. Nachlässe von Gelebrten, Orientalische Handschriften. Handschriften im Besitz von Instituten und Behörden. Register zu Band 1 - 3 (Verzeichniss der Handschriften im preußischen Staate 1, Hannover, 1-3). Berlin: Bath, 1893-1894.

Migne 1865: Jacques Paul Migne, "Circa annum MCCCLV Catalogus librorum

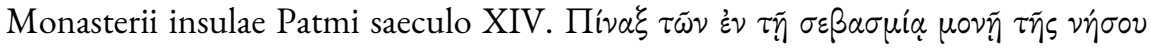

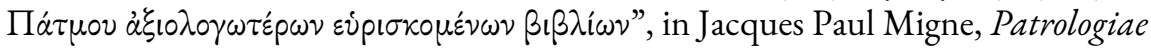
cursus completus, seu bibliotheca universalis, integra, uniformis, commoda, oeconomica, omnium SS. Patrum, Doctorum Scriptorumque Ecclesiasticorum, sive latinorum, sive graecorum ... Series graeca posterior ... Patrologiae graecae tomus 149: Nicephorus Gregoras, Georgius Lapitha, Theodorus Meliteniota. [Parisiis]: apud J.-P. Migne, 1865, cols 1047-1052.

Miller 1848: Emmanuel Miller, Catalogue des mss. grecs de la bibliothèque de l'Escurial. Paris: Imprimerie nationale, 1848.

Miller 1886: Emmanuel Miller, "Bibliothèque royale de Madrid. Catalogue des manuscrits grecs (Supplément au catalogue d'Iriarte)", Notices et extraits des manuscrits de la Bibliothèque nationale et autres bibliothèques 31.2 (1886), pp. 1116.

Minciotti 1842: Luigi Minciotti, Catalogo dei codici manoscritti esistenti nella biblioteca di Sant'Antonio di Padova con brevissimi cenni biografici degli autori. Padova: La Minerva, 1842.

Mittarelli 1779: Johannis-Benedicti Mittarelli, Bibliotheca codicum manuscriptorum Monasterii S. Michaelis Venetiarum prope Murianum una cum Appendice librorum impressorum seculi XV. Opus posthumum. Venetiis: Ex Typographia Femtiana, Sumptibus Praefati Monasterii, 1779.

Modona 1896: Leonello Modona, "La reale biblioteca di Parma", Rivista delle Biblioteche e degli Archivi 6 (1896), pp. 161-168.

Montfaucon 1739: Bernardus De Montfaucon, Bibliotheca Bibliothecarum Manuscriptorum Nova, Ubi, quae innumeris pene manuscriptorum bibliothecis continentur, ad quodvis litteraturae genus spectantia et notatu digna, describuntur et indicantur..., 2 tomi, Parisiis: Apud Briasson, 1739.

Mordtmann 1854: Andreas David Mordtmann, "Verzeichniss der handschriften in der bibliothek sr. maj. des sultans”. Philologus. Zeitschrift fur das klassische Alterthum 9 (1854), pp. 582-584.

Müller 1806-1818: Christian Gottfried Müller, Notitia et Recensio Codicum Mss. qui in bibliotheca episcopatus Numburgo-Cizensis asservantur, particulae 1-8 ... Lipsiae: Ex officina Breitkopfio-Haertelia (particulae 1-4); ex Officina Vogeliana (particulae 5-8), 1806-1818. 
Muralt 1864: Eduard von Muralt, Catalogue des manuscrits grecs de la Bibliothèque impériale publique, volume 1. St.-Pétersbourg: Imprimerie de l'Académie impériale des sciences, 1864 .

Mylius 1746: Iohannes Christophus Mylius, Memorabilia Bibliothecae Academicae Ienensis sive Designatio codicum manuscriptorum in illa bibliotheca et librorum impressorum, plerumque rariorum concinnata potissimum ad usus suorum in collegiis litterariis auditorum. Ienae et Weissenfelsae: apud Ioh. Christoph. Croekerum, 1746.

(Nares) 1808-1812: Robert Nares, A Catalogue of the Harleian Manuscripts in the British Museum with Indexes of Persons, Places, and Matters, 4 volumes. [London] [G. Eyre and A. Strahan], 1808-1812.

Naumann 1838: Aemilius Guilelmus Robertus Naumann, Catalogus librorum manuscriptorum qui in Bibliotheca senatoria Civitatis Lipsiensis asservantur ... Grimae: sumptus fecit Julius Mauritius Gebhardt, 1838.

Neigebauer 1864: Johann Ferdinand Neigebauer, "Die königliche und UniversitätsBibliothek zu Breslau”, Neuer Anzeiger fur Bibliographie und Bibliothekswissenschaft 1864, pp. 7-9, 41-51.

Neigebauer 1865: Johann Ferdinand Neigebauer, "Die Stadtbibliothek in Breslau”, Neuer Anzeiger fur Bibliographie und Bibliothekswissenschaft 1865, pp. 246-253, 293-296.

Nestle 1903: Eberhard Nestle, "Zum Verzeichnis der griechischen Handschriften der K. Universitätsbibliothek in Tübingen”, Centralblatt für Bibliothekswesen 20 (1903), pp. 277-278.

Olivieri, Festa 1895: Alessandro Olivieri, and Nicola Festa, "Indice dei codici greci delle biblioteche Universitaria e Comunale di Bologna”, Studi italiani di Filologia classica 3 (1895), pp. 385-495.

Omont 1883: Henri Auguste Omont, "Inventaire sommaire des manuscrits grecs conservés dans les bibliothèques publiques de Paris autres que la Bibliothèque nationale", Bulletin de la Société de l'Histoire de Paris et de l'Île-de-France 10 (1883), pp. 118-125.

Omont 1885: Henri Auguste Omont, Catalogue des manuscrits grecs de la Bibliothèque royale de Bruxelles et des autres bibliothèques publiques de Belgique. Gand: Imprimerie Eug. Vanderhaeghen, and Paris: Alph. Picard, 1885.

Omont 1886-1888: Henri Auguste Omont, Inventaire sommaire des manuscrits grecs de la Bibliothèque nationale et des autres bibliotbèques de Paris et des Départements, 3 volumes. Paris: Ernest Leroux, 1886-1888.

Omont 1890: Henri Auguste Omont, "Notes sur les manuscrits grecs des villes hanséatiques Hambourg, Brême et Lübeck", Centralblatt für Bibliothekswesen 7 (1890), pp. 351-377. 
Omont 1891: Henri Auguste Omont, "Les manuscrits grecs de la Bibliothèque Capitulaire et de la Bibliothèque Communale de Vérone”, Centralblatt für Bibliothekswesen 8 (1891), pp. 489-497.

Omont 1892: Henri Auguste Omont, "Nouvelles acquisitions du département des manuscrits de la Bibliothèque nationale pendant l'année 1891-1892”, Bibliothèque de l'école des chartes 53 (1892), pp. 333-382.

Omont 1894: Henri Auguste Omont, "Nouvelles acquisitions du département des manuscrits de la Bibliothèque nationale pendant les années 1892-1893", Bibliothèque de l'école des chartes 55 (1894), pp. 61-114, 241-258.

Omont 1896: Henri Auguste Omont, "Nouvelles acquisitions du département des manuscrits de la Bibliothèque nationale pendant les années 1894-1895”, Bibliothèque de l'école des chartes 57 (1896), pp. 161-196; 339-372.

Omont 1897: Henri Auguste Omont, "Complément du catalogue des manuscrits grecs de la Bibliothèque royale de Madrid”, Revue des bibliothèques 7 (1897), pp. $149-154$.

Omont 1898: Henri Auguste Omont, Inventaire sommaire des manuscrits grecs de la Bibliothèque nationale et des autres bibliothèques de Paris et des Départements. Table alphabétique. Paris: Ernest Leroux, 1898.

Omont 1900: Henri Auguste Omont, Nouvelles acquisitions du Départment des Manuscrits pendant l'année 1898-1899. Paris: E. Leroux, 1900.

Omont 1903: Henri Auguste Omont, "Mnauscrits de la bibliothèque de Sir Thomas Phillipps récemment acquis pour la Bibliothèque nationale", Revue des bibliothèques 13 (1903), pp. 189-206.

Omont, Catal. des mss. ... Miller 1897: Henri Auguste Omont, Catalogue des manuscrits grecs, latins, français et espagnols et des portulans recueillis par feu Emmanuel Miller. Paris: Ernest Leroux, 1897.

Omont, Départements 1886: Henri Auguste Omont, Catalogue des manuscrits grecs des départements. Paris: Librarie Plon, E. Plon, Nourrit et C $C^{\text {ie }}, 1886$.

Omont, Pays-Bas 1886: Henri Auguste Omont, "Catalogue des manuscrits grecs des bibliothèques publiques des Pays-Bas (Leyde exceptée)”, Centralblatt für Bibliothekswesen 4 (1886), pp. 185-214.

Omont, Rev. instr. publ. 1884-1885: Henri Auguste Omont, "Catalogue des manuscrits grecs de la Bibliothèque royale de Bruxelles et des autres bibliothèques publiques de Belgique", Revue de l'Instruction Publique (supérieure et moyenne) en Belgique 27 (1884), pp. 311-319, 374-383; 28 (1885), pp. 6-21, 82-89, 168-181.

Omont, Suisse 1886: Henri Auguste Omont, "Catalogue des manuscrits grecs des Bibliothèques de Suisse: Bâle, Einsiedeln, Genève, St Gall, Schaffouse et Zürich”, Centralblatt für Bibliothekswesen 3 (1886), pp. 385-452.

Omont, Supplément Suisse 1891: Henri Auguste Omont, "Supplément au Catalogue des manuscrits grecs des Bibliothèques de Suisse", Centralblatt für Bibliothekswesen 8 (1891), pp. 22-26. 


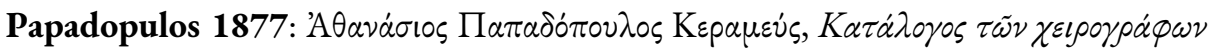

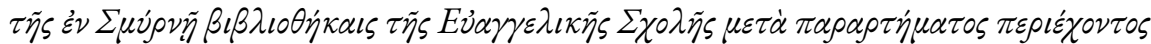

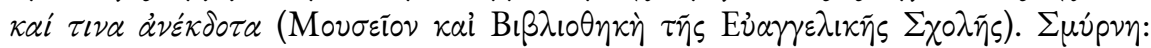

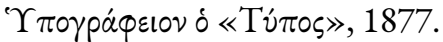

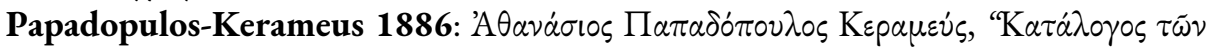

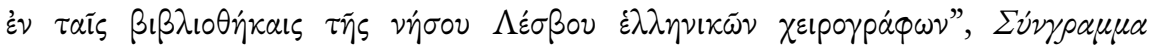

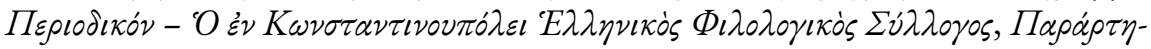

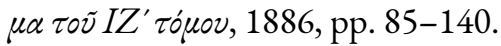

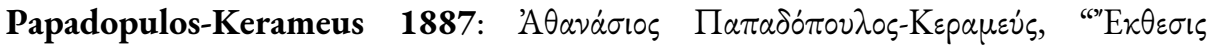

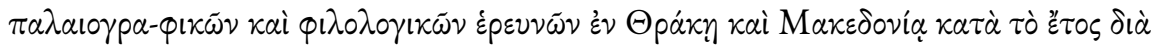

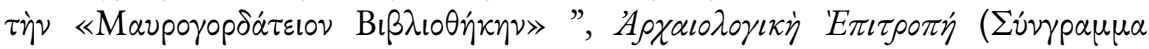

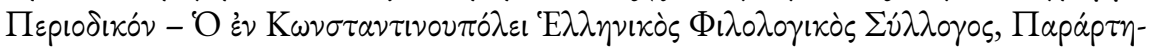

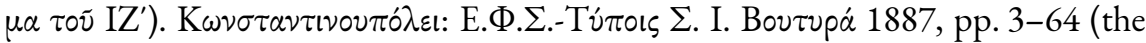

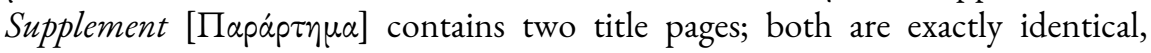
except the year of publication: one [the second] is dated to 1886 and the other [the first] to 1887).

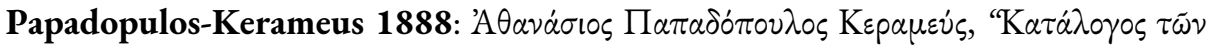

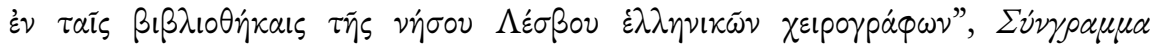

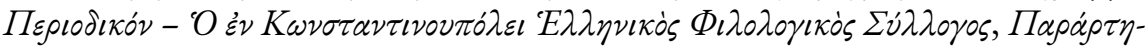

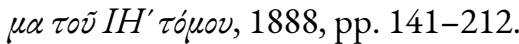

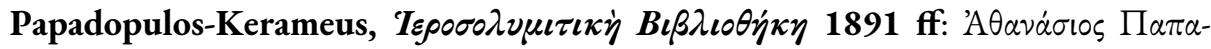

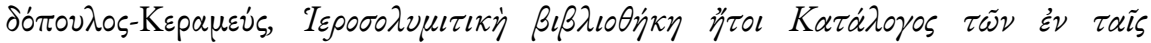

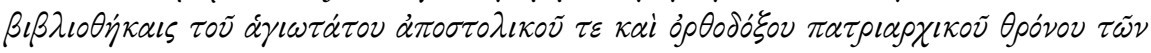

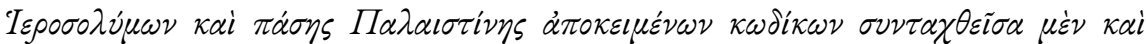

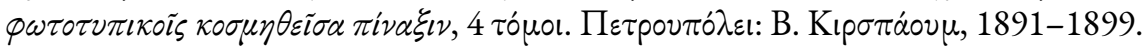

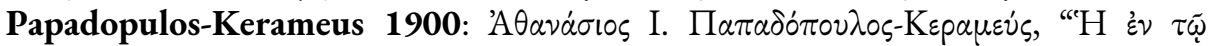

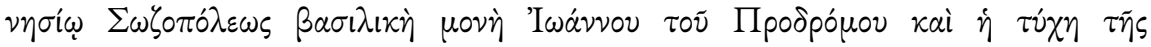

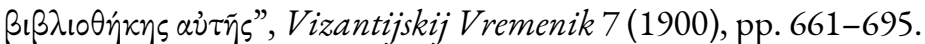

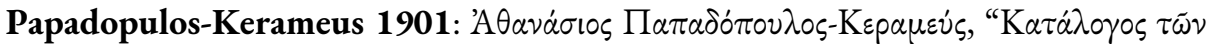

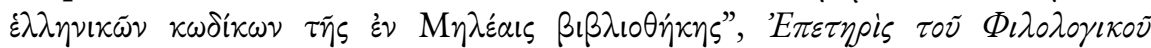

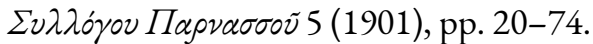

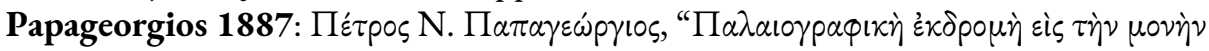

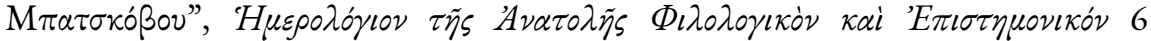
(1887), pp. 115-120.

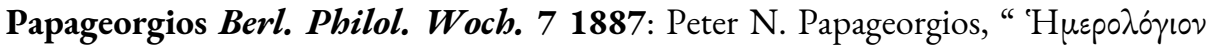

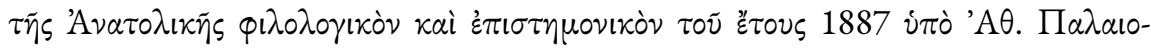

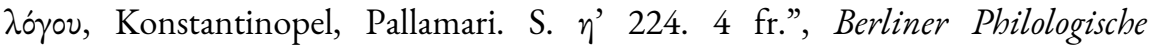
Wochenschrift 7 (1887), cols. 821-823.

Pasinus 1749: Iosephus Pasinus, Antonius Rivautella, and Franciscus Berta, Codices manuscripti Bibliothecae Regii Taurinensis Athenaei Per Linguas digesti, E binas in partes distributi, in quarum Prima Hebraei, E' Graeci, in altera Latini, Italici, 
$\Xi^{2}$ Gallici. Recensuerunt, \& animadversionibus illustrarunt. Taurini: Ex Typographia Regia, 1749.

Passow 1825: Franciscus Passow, Dionysii Orbis Terrarum Descriptio. Recensuit et adnotatione critica instruxit. Accessit tabula geographica lapidi inscripta. Lipsiae: B.G. Teubner, 1825 .

Pélissier 1889: Léon G. Pélissier, "Un inventaire des manuscrits de la Bibliothèque de Corsini à Rome dressé par la Porte du Theil”, Mélanges d'archéologie et d'bistoire de l'École de Rome 9 (1889), pp. 389-429.

Pélissier 1891: Léon G. Pélissier, "Inventaire sommaire de soixante-deux manuscrits de la Bibliothèque Corsini (Rome)”, Centralblatt für Bibliotbekswesen 8 (1891), pp. 176-202, 297-324.

Pervanoglu 1868: Johannes Pervanoglu, "Griechenlands neueste handschriftliche Forschungen”, Neue Jabrbücher für Philologie und Paedagogik 98 (1868), pp. 466-470.

Peyron 1820: Amedeus Peyron, Notitia librorum manu typisve descriptorum qui donante ab. Thoma Valperga-Calusio V. Cl. illati sunt in Reg. Taurinensis Athenaei Bibliothecam bliographica et critica descriptione illustravit anecdota passim inseruit. Lipsiae: Impensis Ioa. Aug. Gottl. Weigelii, 1820.

Pbilolog. 51850 758-62: Friedrich Wilhelm Schneidewin, "Handschriften in Konstantinopel”, Philologus. Zeitschrift fur das klassische Alterthum 5 (1850), pp. $758-760$.

Philologica Hamburgensia 1905: Robert Münzel (ed.), Philologica Hamburgensia für die Mitglieder der 48. Versammlung deutscher Philologen und Schulmänner ausgestellt von der Stadtbibliothek zu Hamburg. Hamburg: Lütcke und Wulff, 1905.

Piccolomini 1898: Aeneas Piccolomini, "Index codicum graecorum Bibliothecae Angelicae ad praefationem additamenta”, Studi italiani di fllologia classica 6 (1898), pp. 167-184.

Pierleoni 1901: Gino Pierleoni, "Index codicum graecorum qui Romae in Bybliotheca Corsiniana nunc Lynceorum adservantur”, Studi italiani di filologia classica 9 (1901), pp. 467-478.

Porro 1884: Giulio Porro, Catalogo dei codici manoscritti della Trivulziana (Biblioteca storica, Reale Deputazione di Storia patria 2). Torino: Fratelli Bocca (Stamperia Reale), 1884.

Puntoni 1896/1: Vittorio Puntoni, "Indice dei codici greci della Biblioteca Estense di Modena", Studi italiani di filologia classica 4 (1896), pp. 379-536.

Puntoni 1896/2: Vittorio Puntoni, "Indicis codicum graecorum Bononiensium ab Al. Oliverio compositi supplementum”, Studi italiani di filologia classica 4 (1896), pp. 365-378.

Rocchi 1883: Antonius Rocchi, Codices cryptenses seu Abbatiae Cryptae Ferratae in Tusculano digesti et illustrati. Romae: Ex Typographia Pacis, Philippi Cuggiani, 1883. 
Roscoe 1815-1828: William Roscoe, Catalogue of manuscripts in the library at Holkham in the County of Norfolk, belonging to Thomas William Coke, Esq., M.P. Compiled and arranged by William Roscoe; collated and enlarged by Frederic Madden, 8 volumes, 1815-1828.

Rostagno 1894: Enrico Rostagno, “Codici greci Laurenziani meno noti”, Studi italiani di filologia classica 2 (1894), p. 154.

Rostagno 1898: Enrico Rostagno, "Indicis codicum graecorum bybliothecae Laurentianae supplementum”, Studi italiani di filologia classica 6 (1898), pp. 129-166.

Rostagno-Festa 1893: Enrico Rostagno e Nicola Festa, "Indice dei codici greci Laurenziani non compresi nel catalogo del Bandini”, Studi italiani di filologia classica 1 (1893), pp. 129-232.

Ruelle 1875: Charles Emile Ruelle, "Rapports sur une mission littéraire et philologique en Espagne", Archives des missions scientifiques et littéraires. Choix de rapports et instructions publié sous les auspices du Ministère de l'instruction publique et des beaux-arts, $3^{\mathrm{e}}$ série, tome 2 (1875), pp. 497-627.

Rühl 1889: Franz Rühl, "Bemerkungen über einige Bibliotheken von Sicilien”, Philologus. Zeitschrift fur das klassische Alterthum N. F. $1=47$ (1889), pp. 577588.

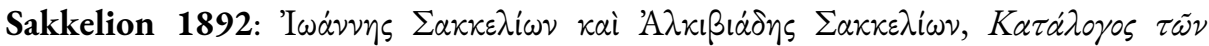

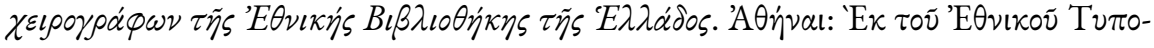

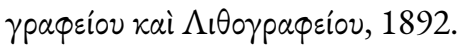

Schenkl 1896: Heinrich Schenkl, Bibliotheca patrum latinorum Britannica. VII (Sitzungsberichte der kaiserlichen Akademie der Wissenschaften, Philosophishhistorische Classe 133 [1895], Abhandlung 7). Wien: Kaiserliche Akademie der Wissenschaften, 1896.

Schmid 1902: Wilhelm Schmid, Verzeichnis der griechischen Handschriften der königlichen Universitätsbibliothek zu Tübingen. Tübingen: Buchdruckerei von G. Schnürlen, 1902.

Schmid 1903: Wilhelm Schmid, "Zum Verzeichnis der Tübinger griechischen Handschriften”, Centralblatt für Bibliothekswesen 20 (1903), pp. 462-464.

Schneider 1840/41: Karl Ernst Christoph Schneider, "Descriptio duorum codicum MSS. Galeni libros latinam in linguam translatos continentium”, in Index lectionum in Universitate Litterarum Vratislaviensi per hiemem Anni MDCCCXL. a Die XIX M. Octobris instituendarum. Vratislavae: Universitas Literarum Vratislaviensis, $1840 / 41$, pp. 1-14.

Schnorr v. Carolsfeld 1882-1883: Franz Schnorr von Carolsfeld, Katalog der Handschriften der Königl. öffentlichen Bibliothek zu Dresden. Im Auftrage der Generaldirection der königlichen Sammlungen für Kunst und Wissenschaft bearbeitet von -. Erster Band. (enthaltend die Abtheilungen A-D und F-H). Zweiter Band. (enthaltend die Abteilungen J-M). Leipzig: Druck und Verlag von B. G. Teubner, 1882-1883. 
Scott 1904: Edward J. L. Scott, Index to the Sloane manuscripts in the British Museum. London: British Museum, 1904.

Senebier 1779: Jean Senebier, Catalogue raisonné des manuscrits conservés dans la Bibliothèque de la Ville et République de Genève. Genève: Barthelemy Chirol, 1779.

Sinker 1891: Robert Sinker, The Library of Trinity College, Cambridge. Cambridge: Deighton, Bell and Co., and London: George Bell and Sons, 1891.

Smith 1849: John James Smith, A Catalogue of the Manuscripts in the Library of Gonville and Caius College, Cambridge. Cambridge: J. Deighton, and Macmillan \& Co.; London: John W. Parker; Oxford: J. Parker, 1849.

Sparfwenfeldt 1706: Ioan. Gabr. Sparvenfeldius, Catalogus centuriae librorum rarissimorum manuscript. Et partim impressorum, Arabicorum, Persicorum, Turcicorum, Graecorum, Latinorum, etc. Qua anno MDCCV Bibliothecam Publicam Academiae Upsalensis auxit et exornavit Vir illustris et generosissimus ... Upsaliae: Typis Joh. Henr. Werneri, 1706.

Statistica del regno d'Italia 1865: Statistica del regno d'Italia. Biblioteche. Anno 1863. Firenze: Tipografia dei successori Le Monnier, 1865.

Statistica delle biblioteche 1893-1894: Ministero di Agricoltura, Industria e Commercio (Direzione generale della statistica), Statistica delle biblioteche. Parte I. Biblioteche dello stato, delle provincie, dei comuni ed altri enti morali aggiuntevi alcune biblioteche private accessibili agli studiosi, tra le più importanti per numero di volumi o per rarità di collezioni. Volume I. Piemonte, Liguria, Lombardia, Veneto ed Emilia; Volume II. Toscana, Marche, Umbria, Roma, Abruzzi e Molise, Campania, Puglie, Basilicata, Calabria, Sicilia e Sardegna. Roma: Tipografia nazionale di G. Bertero, 1893-1894.

Stevenson 1885: Henry Stevenson, Codices manuscripti Palatini graeci Bibliothecae Vaticanae descripti. Romae: Ex Typographeo Vaticano, 1885.

Stevenson 1888: Henry Stevenson, Bibliothecae Apostolicae Vaticanae ... Codices Reginae Suecorum et Pii PP. II graeci. Romae: Ex Typographeo Vaticano, 1888.

Stornajolo 1895: Cosimo Stornajolo, Codices urbinates graeci Bibliothecae Vaticanae descripti ... Accedit Index vetus Bibliothecae urbinatis nunc primum editus. Romae: Ex Typographeo Vaticano, 1895.

Studemund-Cohn 1890: Wilhelm Studemund und Leopold Cohn, Codices ex Bibliotheca Meermanniana Phillippici Graeci nunc Berolinenses (Verzeichniss der griechischen Handschriften der königlichen Bibliothek zu Berlin 1). Berolini: A. Asher \& Co, 1890.

Svedelius 1806: Pehr Fabian Aurivillius, Notitia codicum manuscriptorum Grecorum biblioth. acad. Upsaliensis. Quam consensu ampliss. fac. phil. Ups. preside Petro Fabiano Aurivillio ... pro gradu philosopbico publico examini subjicit Petrus Gustavus Svedelius, Dalekarlus. In audit. Gust. maj. d. VI. Junii MDCCCVI ... Upsaliæ: typis Edmannianis, [1806]. 
Szilágyi 1881-1894: Sándor Szilágyi, Catalogus manuscriptorum Bibliotbecae Regiae Scientiarum Universitatis Budapestinensis, 3 volumes. Budapestini: Typis Typographiae Universitatis Regiae Scientiarum, 1881-1894.

The Academy 1880: Greville John Chester, "The Greek Library at Smyrna", The Academy - A Weekly Review of Literature Science, and Arts 17 (January-June 1880), p. 178.

Thompson 1881-1884: [Edward Maunde Thompson, and George F. Warner], Catalogue of Ancient Manuscripts in the British Museum, 2 volumes. London: British Museum, 1881-1884.

Thraemer 1892: Eduard Thrämer, "Auf der Suche nach der Bibliothek Iwans des Schrecklichen”, Allgemeine Zeitung, Beilage, 1892, Heft 1, pp.4-7; Heft 2, pp. 1-4; Heft 3, pp. 3-5.

Todd 1812: Henry John Todd, $A$ catalogue of the archiepiscopal manuscripts in the library at Lambeth Palace. With an account of the archiepiscopal registers and other records there preserved. London: Law and Gilbert, 1812.

Tomasini 1639: Iacobus Philippus Tomasini, Bibliothecae patavinae manuscriptae publicae et privatae. Utini: Typis Nicolai Schiratti, 1639.

Tomasini 1650: Iacobus Philippus Tomasini, Bibliothecae venetae manuscriptae publicae et privatae. Utini: Typis Nicolai Schiratti, 1650.

Van den Gheyn 1901-1904: Joseph Van den Gheyn, Catalogue des manuscrits de la Bibliothèque Royale de Belgique, vol. 1: Écriture sainte et liturgie; vol. 2: Patrologie; vol. 3: Théologie; vol. 4: Jurisprudence et philosophie, Brussels: Lambertin, 19011904 .

Vater 1843: Friedrich Vater, "Zur Kunde griechischer Handschriften in Russland", Neue Jabrbücher für Philologie und Paedagogik oder Kritische Bibliothek für das Schul-und Unterrichtswesen 9. Supplementband (1843), pp. 5-49.

Vitelli 1894: Girolamo Vitelli, “Indice de' codici greci Riccardiani, Magliabechiani e Marucelliani”, Studi italiani di filologia classica 2 (1894), pp. 471-570.

Vogel 1841-1842: Ernst Vogel, "Ueber einige frühere italienische Bibliotheken”, Serapeum - Zeitschrift für Bibliothekswissenschaft, Handschriftenkunde und ältere Literatur 2 (1841), pp. 24-32, 41-46, 52-59, 72-79, 318-319, 321-330; 3 (1842), pp. 140-144.

Volger 1858: Ernst Volger, "Mittheilungen aus handschriften”, Philologus. Zeitschrift für das klassische Alterthum 13 (1858), pp. 192-195.

Volger 1859: Ernst Volger, "Mittheilungen aus Spanien”, Philologus. Zeitschrift für das klassische Alterthum 14 (1859), pp. 161-168.

Volger, Philologus 14 1859: Ernst Volger, "Mittheilungen aus Spanien. 23. Verzeichniss der classischen HSS. der Universitätsbibliothek von Salamanca”, Philologus. Zeitschrift für das klassische Alterthum 14 (1859), pp. 373-379. 
v. Gebhardt 1898: Oscar von Gebhardt, "Christian Friedrich Matthaei und seine Sammlung griechischer Handschriften. II. Zur Orientierung über die Moskauer Bibliotheken", Centralblatt für Bibliothekswesen 15 (1898), pp. 393-420.

v. Heinemann 1884-1888: Otto von Heinemann, Die Handschriften der Herzoglichen Bibliothek zu Wolfenbüttel. Erste Abteilung. Die Helmstedter Handschriften, 3 volumes. Wolfenbüttel: Druck und Verlag Julius Zwissler, 1884-1888.

v. Heinemann 1890-1903: Otto von Heinemann, Die Handschriften der Herzoglichen Bibliothek zu Wolfenbüttel. Zweite Abteilung. Die Augusteischen Handschriften. 5 volumes. Wolfenbüttel: Julius Zwissler, 1890-1903.

v. Heinemann 1903: Otto von Heinemann, Die Handschriften der Herzoglichen Bibliothek zu Wolfenbüttel. Dritte Abteilung. Die Weissenburger Handschriften. Wolfenbüttel: Julius Zwissler, 1903.

Wachler 1828: Albrecht W. J. Wachler, Thomas Rhediger und seine Büchersammlung in Breslau. Ein biographisch-literärischer Versuch. Breslau: Grüson,1828.

Wegener 1876: Philipp Wegener, "Verzeichnis der auf der Zeitzer Stiftsbibliothek befindlichen Handschriften”, Programm des Königlichen Stifts-Gymnasiums in Zeitz ... (Programm 206). Zeitz: 1876, pp. 1-22.

Weidmann 1841: Franz Weidmann, "Verzeichniss der im Jahre 1712 nach Zürich abgeführten und nicht zurückgegebenen sanctgallischen Handschriften”, in Weidmann, Geschichte der Bibliothek von St. Gallen seit ibrer Gründung um das Jabr 830 bis auf 1841. Aus den Quellen bearbeitet auf die Tausendjäbrige Jubelfeier. St. Gallen: Scheitlin und Zollikofer, 1841, pp. 423-441.

Weinberger 1902: Wilhelm Weinberger, Catalogus catalogorum. Verzeichnis der Bibliotheken, die ältere Handschriften lateinischer Kirchenschriftsteller enthalten im Auftrage cer Kaiserlichen Akademie der Wissenschaften in Wien zusammengestellt. Prag: F. Tempsky; Wien: F. Tempsky; Leipzig: G. Freitag, 1902.

Weißenborn 1857: Hermann Johann Christian Weißenborn, “Die Bibliotheken zu Constantinopel und deren alte Handschriften”, Neue Jabrbücher für Philologie und Paedagogik 76 (1857) pp. 201-203.

Wilken 1817: Friedrich Wilken, Geschichte der Bildung, Beraubung und Vernichtung der alten Heidelbergischen Büchersammlungen: ein Beytrag zur Literärgeschichte vornebmlich des funfzebnten und sechszehnten Jabrbunderts; nebst einem meist beschreibenden Verzeichniß der im Jabr 1816 von dem Papst Pius VII. der Universität Heidelberg zurückgegebenen Handschriften und einigen Schriftproben. Heidelberg: August Oswald's Universitäts-Buchhandlung, 1817.

Wolf 1764: Johann David Wolf, Compendiaria Bibliothecae Neapolitanae Vratislaviensis ad Aedem S. Bernardini servatae descriptio ... Bregae: Gottfr. Boerner, 1764.

[Zanetti] 1740: Anton Maria Zanetti and Antonio Bongiovanni, Graeca D. Marci Bibliotheca codicum manuscriptorum per titulos digesta ... Venetiis: apud Simonem Occhi, 1740. 
clxxxvi

Zentralbl. f. Bibl. Wesen 19 1902: Karl Konrad Müller, "Besichtigung der Universitätsbibliothek und ihrer Cimelien mit einleintenden Worten des Direktors"; "Die Ausstellung auf der Universitätsbibliothek in Jena gelegentlich der Bibliothekarversammlung (S. 384) umfasste von Hss.”, Centralblatt für Bibliothekswesen 19 (1902), pp. 380-384, 428-429.

Zuretti 1896: Carlo Oreste Zuretti, "Indice de' mss. greci torinesi non contenuti nel catalogo del Pasini”, Studi italiani di Filologia classica 4 (1896), pp. 201-223. 


\section{Editions of Anonymous Greek Medical Texts}

This bibliography of printed anonymous Greek medical texts provides references to the edition of ca. fifty anonymous texts found in manuscripts and papyri. Although it is not comprehensive, in a certain way it complements the lists of manuscripts by authors and texts which make up the substance of Diels.

Though not explicitly divided into two parts, this bibliography comprises two sections: texts in manuscripts and texts in papyri (in this order). These two parts are clearly distinguished here. In both, items are identified as they are in Diels, by the title of the text (in many cases in Greek) or by another type of title (from an edition or from the scholarly tradition). These titles are exactly reproduced here in boldface for purposes of clarity. They are listed in alphabetical order and followed by the reference to the edition(s) listed in Diels. Whereas in Diels, these references are mostly in an abbreviated form, they are fully expanded here, and formatted according to standard bibliographical practice. In several cases, more than one edition is mentioned in Diels. All are listed here in chronological order. In some cases, such different editions are distinguished in Diels by textual characteristics (e.g., the incipit of the text). All these distinctive elements are exactly reproduced here.

In some cases, new elements of identification of the works referred to are added here (for instance, the exact pages where the edition of the text can be read). 



\section{Texts from manuscripts}

Anonymi metrologici: Friedrich Hultsch, Metrologicorum scriptorum reliquiae, collegit, recensuit, partim nunc primum edidit -, volumen I. Lipsiae: B.G. Teubner, 1864 .

Carmen de viribus herbarum: Moritz Haupt, Index lectionum quae auspiciis Regis augustissimi Guilelmi Imperatoris germanici in universitate litterarum Frideria Guilelma per semestre hibernum a. d. XVI. m. octobris A. MDCCCLXXIII usque ad d. XXII. m. martii MDCCCLXXIV habentur. Berolini: Formis Academicis (G. Vogt), 1873-1874. Inc.: Carmen graecum de viribus herbarum quod dicitur ...

Vgl. Mauricius Hauptius, Opuscula, volumen secundum: Prooemia indicibus lectionum praefixa. Lipsiae: Salomon Hirzelius, 1876, pp. 475-489: "Index Lectionum Hibernarum 1873”. Inc.: Carmen Graecum de viribus herbarum ...

Georg Kaibel, "Sententiarum Liber Quintus”, Hermes. Zeitschrift für classische Philologie 25 (1890), pp. 97-112 (see section VII, pp. 103-109).

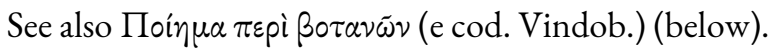

Fragmentum medicum (aus cod. bibl. Lips. 175 f. 17): Conrad Bursian, "Fragmentum medicum Graecum", in Index scholarum publice et privatim in Universitate Litterarum Ienensi inde a Die XX M. Octobris A. MDCCCLXXXIII usque ad Diem XXI M. Martii A. MDCCCLXXIIII habendarum. Ienae: Officina Neuenhahni, 1873, pp. 3-14.

Laterculi medicorum graecorum: bei Otto Kröhnert, Canonesne poetarum, scriptorum artificum per antiquitatem fuerunt? Dissertatio inauguralis philologa quam consensu et auctoritate amplissimi philosophorum ordinis in Alma Litterarum Universitate albertina Regimontana ad summos in philosophia honores rite capessendos die XXVIII. mensis Iunii. a. MDCCCXCVII ... Regimonti Prussorum: Officina Leopoldiana, 1897, esp. pp. 54-63.

Laterculi medicorum graecorum et latinorum (cod. Laur. lat. 73, 1): bei Max Wellmann, "Zur Geschichte der Medizin im Alterthum" Hermes. Zeitschrift für classische Philologie 35 (1900), pp. 349-384 (see especially pp. 367-382, section VI about the text on $\mathrm{ff} .142^{\mathrm{v}}-143^{\mathrm{r}}$ of the manuscript).

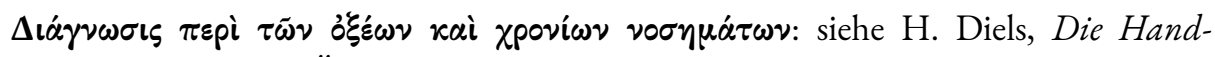
schriften der antiken Ärzte II, p. 48 s. v. Herodotus. 


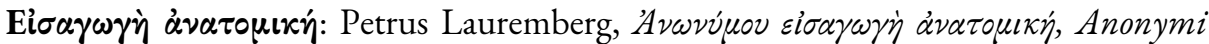
philosophi antiquiss: Isagoge Anatomica. Nunc primùm è suâ Bibliotheca edidit, \& vertit -. Hamburgi: Paulus Langius, 1616.

edd. Daniel Wilhelm Triller et Johannes Stephanus Bernard, Anonymi Introductio Anatomica Gr. et Lat. Item Hypatus De partibus corporis, Gr. et Lat. cum notis -. Accedunt Figurae Anatomicae cum explicatione Graeca, nunc primum ex Codice MS. Bibliothecae Leidensis editae. Lugduni Batavorum: Philippus Bonk, 1744, pp. 1-141.

$V g l$. Wilhelm Schmidt, De Anonymi Laurembergiani introductione anatomica. Dissertatio inauguralis quam ad summos in philosophia honores impetrandos consensu et auctoritate amplissimi philosophorum ordinis in Alma Litterarum Universitate Friderica Guilelma Berolinensi scripsit -. Berolini: Noske Bornensis, 1905.

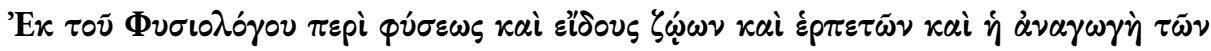

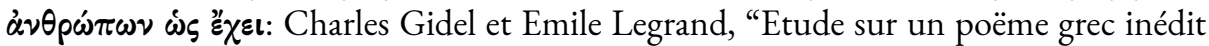
intitulé O $\Upsilon \Upsilon \Sigma \mathrm{IO} \Lambda \mathrm{O} \mathrm{O} \Sigma$ ”, Annuaire de l'association pour l'encouragement des études grecques 7 (1873), pp. 188-296 (edition of the Greek text by Legrand, pp. 225-286).

$V g l$. Karl Krumbacher, Geschichte der byzantinischen Litteratur von Justinian bis zum Ende des oströmischen Reiches (527-1453), 2. Auflage, bearbeitet unter Mitwirkung von Albert Ehrhard und Heinrich Gelzer. München: C. H. Beck'sche Verlagsbuch handlung, 1897, pp. 874-877.

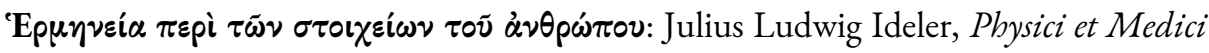
graeci minores. Congessit, ad fidem codd. MSS. praesertim eorum, quos beatus Dinzius contulerat, veterumque editionum partim emendavit partim nunc prima vice edidit, commentatiis criticis indicibusque tan rerum quam verborum instruxit, 2 volumes. Berolini: G. Reimer, 1841-1842, vol. 1, pp. 301-302.

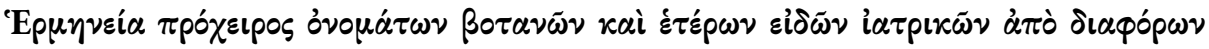

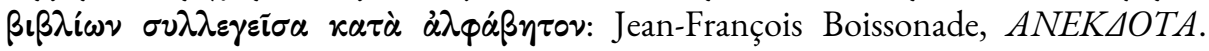
Anecdota graeca e codicibus regiis descripsit annotatione illustravit, vol. II. Parisiis: In Regio Typographeo, 1830, pp. 394-408.

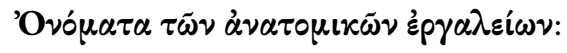

Friedrich Reinhold Dietz, Severi sophistae de clysteribus liber ad fidem cod. manuscripti unici Florentini primum graece editus. Regimontii Prussorum: apud fratres Borntraeger, 1836, pp. 46-48. 
Hermann Schoene, "Zwei Listen chirurgische Instrumente”, Hermes. Zeitschrift für classische Philologie 38 (1903), pp. 280-284.

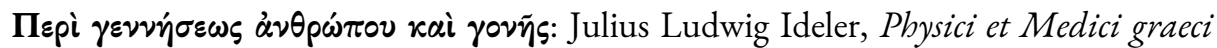
minores. Congessit, ad fidem codd. MSS. praesertim eorum, quos beatus Dinzius contulerat, veterumque editionum partim emendavit partim nunc prima vice edidit, commentatiis criticis indicibusque tan rerum quam verborum instruxit, 2 volumes. Berolini: G. Reimer, 1841-1842, vol. 1, pp. 294-296.

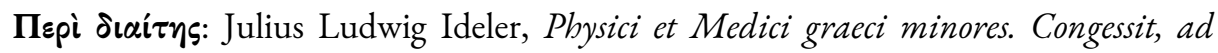
fidem codd. MSS. praesertim eorum, quos beatus Dinzius contulerat, veterumque editionum partim emendavit partim nunc prima vice edidit, commentatiis criticis indicibusque tan rerum quam verborum instruxit, 2 volumes. Berolini: G. Reimer, 1841-1842, vol. 2, pp. 194-198.

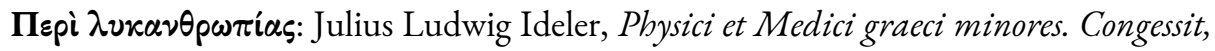
ad fidem codd. MSS. praesertim eorum, quos beatus Dinzius contulerat, veterumque editionum partim emendavit partim nunc prima vice edidit, commentatiis criticis indicibusque tan rerum quam verborum instruxit, 2 volumes. Berolini: G. Reimer, 1841-1842, vol. 2, p. 282.

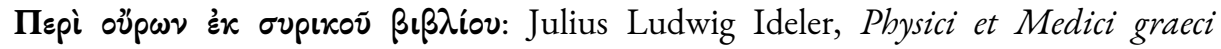
minores. Congessit, ad fidem codd. MSS. praesertim eorum, quos beatus Dinzius contulerat, veterumque editionum partim emendavit partim nunc prima vice edidit, commentatiis criticis indicibusque tan rerum quam verborum instruxit, 2 volumes. Berolini: G. Reimer, 1841-1842, vol. 2, pp. 303-304.

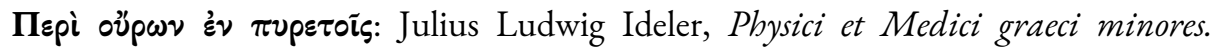
Congessit, ad fidem codd. MSS. praesertim eorum, quos beatus Dinzius contulerat, veterumque editionum partim emendavit partim nunc prima vice edidit, commentatiis criticis indicibusque tan rerum quam verborum instruxit, 2 volumes. Berolini: G. Reimer, 1841-1842, vol. 2, pp. 323-327.

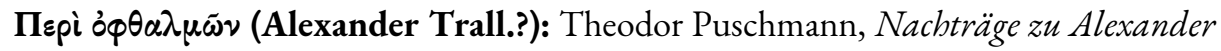
Trallianus. Fragmente aus Philumenus und Philagrius, nebst einer Bisher noch ungedruckten Abbandlung über Augenkrankbeiten. Nach den Handschriften herausgegeben und ins Deutsche übersetzt (Berliner Studien für classische Philologie und Archaeologie 5. Band, 2. Heft). Berlin: S. Calvary \& Co., 1886, pp. 130-179.

Vgl. Georg Helmreich, "Zum Anonymus Пвpi ò $\varphi \theta \alpha \lambda \mu \tilde{\omega \nu}$ ”, Philologus. Zeitschrift für das klassische Alterthum 51 (N.F. 5) (1892), p. 746. 
Пврі $\pi \alpha \theta \tilde{\omega} \nu$ : Richard Schneider, Excerpta $\pi \varepsilon \rho i \pi \alpha \theta \tilde{\omega} \nu$ (Beigabe zu den Jahresbericht des Königlichen Gymnasiums zu Duisburge 1895, Programm Nummer 446). Leipzig: B. G. Teubner, 1895.

Пвpi $\sigma \varphi v \gamma \mu \tilde{\omega} v:$ Julius Ludwig Ideler, Physici et Medici graeci minores. Congessit, ad fidem codd. MSS. praesertim eorum, quos beatus Dinzius contulerat, veterumque editionum partim emendavit partim nunc prima vice edidit, commentatiis criticis indicibusque tan rerum quam verborum instruxit, 2 volumes. Berolini: G. Reimer, 1841-1842, vol. 2, p. 317.

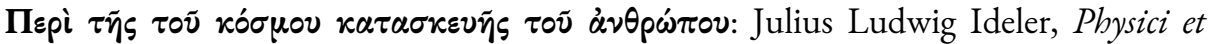
Medici graeci minores. Congessit, ad fidem codd. MSS. praesertim eorum, quos beatus Dinzius contulerat, veterumque editionum partim emendavit partim nunc prima vice edidit, commentatiis criticis indicibusque tan rerum quam verborum instruxit, 2 volumes. Berolini: G. Reimer, 1841-1842, vol. 1, pp. 303-304.

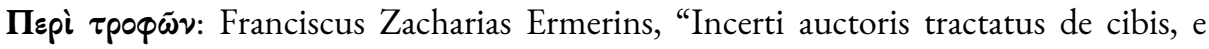
libro de medicina ad Costantinum Pogonatum", in Idem, Anecdota medica graeca. Lugduni Batavorum: Luchtmans, 1840, pp. 223-275 (actual Greek title: 'Eל i i $\tau$ p $к \tilde{\eta} s$

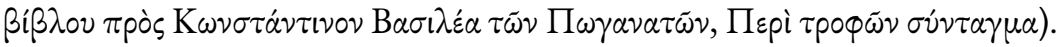

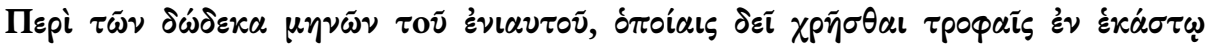

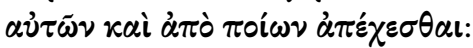

Jean-François Boissonade, ANEKAOTA. Anecdota graeca e codicibus regiis descripsit annotatione illustravit, vol. III. Parisis: In Regio Typographeo, 1830, pp. 409-421.

Julius Ludwig Ideler, Physici et Medici graeci minores. Congessit, ad fidem codd. MSS. praesertim eorum, quos beatus Dinzius contulerat, veterumque editionum partim emendavit partim nunc prima vice edidit, commentatiis criticis indicibusque tan rerum quam verborum instruxit, 2 volumes. Berolini: G. Reimer, 18411842, vol. 2, pp. 423-429.

\section{Пepì vंdpoфoßías:}

ed. James Sims, De hydrophobia. Graece et latine. Interprete -. [Arnhem: W. Troost, 1791].

Reproduction of Sims' edition and translation (originally published in the Memoirs of the Medical Society of London 2 [1789], pp. 2-9 under the title "Of the Hydrophobia, from a Greek manuscript, in the possession James Sims ... with 
a translation by the fame”) by Jo. Steph. Bernard, with Sims' Greek and Latin text pp. 1-10, and Bernard's commentary pp. 11-31.

Christianus Gothfr. Gruner, Ioannis Stephani Bernard Reliquiae medico-criticae edidit -. Ienae: [s.n.], 1795. See pp. 54-116 (reproduction of the work above, originally printed in a limited number of copies).

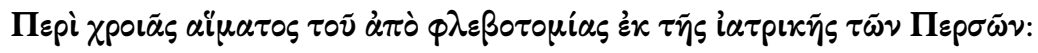

Christian Godofredus Gruner, "Anonymi fragmentum de venae sectione”, in Ordinis medici in Universitate Litteraria Ienensi H. T. Decanus D. Christ. Godofred. Gruner ... sollemnia inauguralia viri praenobilissimi atque experientissimi Frederici Hoffmann ... A. D. XVIIII. August. MDCCLXXX. indicit. Ienae: Litteris Fickelscherrii, 1780, pp. 3-13.

Julius Ludwig Ideler, Physici et Medici graeci minores. Congessit, ad fidem codd. MSS. praesertim eorum, quos beatus Dinzius contulerat, veterumque editionum partim emendavit partim nunc prima vice edidit, commentatiis criticis indicibusque tan rerum quam verborum instruxit, 2 volumes. Berolini: G. Reimer, 18411842, vol. 1, p. 293.

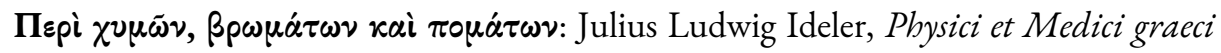
minores. Congessit, ad fidem codd. MSS. praesertim eorum, quos beatus Dinzius contulerat, veterumque editionum partim emendavit partim nunc prima vice edidit, commentatiis criticis indicibusque tan rerum quam verborum instruxit, 2 volumes. Berolini: G. Reimer, 1841-1842, vol. 2, pp. 257-281.

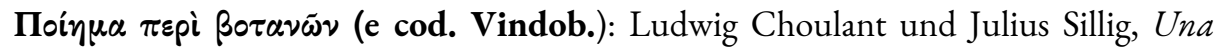
cum Walafridi Strabonis Othonis Cremonensis et Ioannis Folcz Carminibus similis argumenti quae secundum codices manuscriptos et veteres editiones recensuit, supplevit et adnotatione critica instruxit L. C. Accedit anonymi carmen graecum de herbis quod e codice vindobonensi auxit et cum Goddfredi Hermanni suisque emendationibus ediditJ. S. Lipsiae: Leopoldi Vossii, 1832, pp. 195-216.

\section{$\Sigma \tau^{\prime} \chi \chi \iota \iota \tilde{\omega} \nu$ เ $\beta^{\prime} \mu \eta \nu \tilde{\omega} \nu$ (e cod. Paris. 2286. f. 124):}

Jean-François Boissonade, "Traité alimentaire du médecin Hérophile extrait de deux manuscrits de la bibliothèque du roi", Notices et Extraits des manuscrits de la bibliothèque du roi et autres bibliothèques 11 (1827), seconde partie, pp. 178-273.

Julius Ludwig Ideler, Physici et Medici graeci minores. Congessit, ad fidem codd. MSS. praesertim eorum, quos beatus Dinzius contulerat, veterumque editionum partim emendavit partim nunc prima vice edidit, commentatiis criticis indicibus- 
que tan rerum quam verborum instruxit, 2 volumes. Berolini: G. Reimer, 18411842, vol. 1, pp. 421-422.

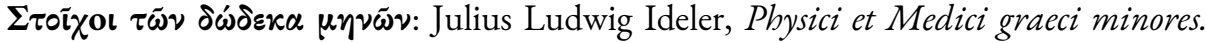
Congessit, ad fidem codd. MSS. praesertim eorum, quos beatus Dinzius contulerat, veterumque editionum partim emendavit partim nunc prima vice edidit, commentatiis criticis indicibusque tan rerum quam verborum instruxit, 2 volumes. Berolini: G. Reimer, 1841-1842, vol. 1, pp. 299-300.

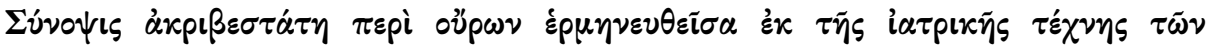

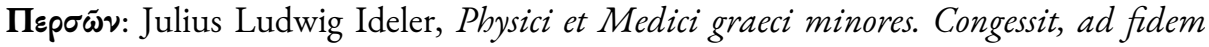
codd. MSS. praesertim eorum, quos beatus Dinzius contulerat, veterumque editionum partim emendavit partim nunc prima vice edidit, commentatiis criticis indicibusque tan rerum quam verborum instruxit, 2 volumes. Berolini: G. Reimer, 1841-1842, vol. 2, pp. 305-306.

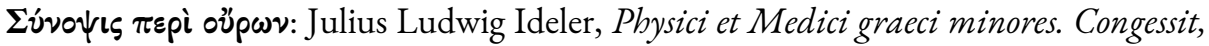
ad fidem codd. MSS. praesertim eorum, quos beatus Dinzius contulerat, veterumque editionum partim emendavit partim nunc prima vice edidit, commentatiis criticis indicibusque tan rerum quam verborum instruxit, 2 volumes. Berolini: G. Reimer, 1841-1842, vol. 2, pp. 307-316. 


\section{Texts from papyri}

A medical papyrus fragment: Edgar Johnson Goodspeed, "A Medical Papyrus Fragment”, American Journal of Philology 24 (1903), pp. 327-329.

Anonymi Londinensis ex Aristotelis Iatricis Menoniis et aliis medicis eclogae: Hermann Diels, Anonymi Londinensis ex Aristotelis Iatricis Menoniis et aliis medicis eclogae (Supplementum Aristotelicum voluminis III pars 1). Berolini: G. Reimer, 1893.

Papyri Argentoratenses graecae (Remedia oculorum. De febribus): Karl Kalbfleisch, Papyri Argentoratenses graecae editae (Ad Scholas quae in has Universitate Rostochiensi per semestre aestivum inde a D. XV M. Aprilis usque a D.XV M. Augusti Z. MDCCCI habebuntur invitant Rector et Concilium). Rostochii: Typis academicis Adlerianis, 1901, pp. 3-12.

Papyrus Berolinensis 7763. 9776 (Medicinische Recepte): ed. Hermann Schoene, in Karl Kalbfleisch und Hermann Schöne (herausgegeben von -), Berliner Klassikertexte, Heft III, Griechische Papyri medizinischen und naturwissenschaftlichen Inbalts. Berlin: Weidmannsche Buchhandlung, 1905, pp. 32-33, 33-34.

Papyrus Berolinensis 9015 (Lehre der empirischen Ärzte): ed. Hermann Schoene, in Karl Kalbfleisch und Hermann Schöne (herausgegeben von -), Berliner Klassikertexte, Heft III, Griechische Papyri medizinischen und naturwissenschaftlichen Inbalts. Berlin: Weidmannsche Buchhandlung, 1905, pp. 29-30.

Papyrus Berolinensis 9095 (Behandlung einer Verstopfung): ed. Karl Kalbfleisch, in Karl Kalbfleisch und Hermann Schöne (herausgegeben von -), Berliner Klassikertexte, Heft III, Griechische Papyri medizinischen und naturwissenschaftlichen In halts. Berlin: Weidmannsche Buchhandlung, 1905, pp. 19-21.

Papyrus Berolinensis 9764 (Medizinischer Unterricht): ed. Hermann Schoene, in Karl Kalbfleisch und Hermann Schöne (herausgegeben von -), Berliner Klassikertexte, Heft III, Griechische Papyri medizinischen und naturwissenschaftlichen Inbalts. Berlin: Weidmannsche Buchhandlung, 1905, pp. 22-26.

Papyrus Berolinensis 9765 (Gewinnung und Reinigung von Rindstalg): ed. Hermann Schoene, in Karl Kalbfleisch und Hermann Schöne (herausgegeben von -), Berliner Klassikertexte, Heft III, Griechische Papyri medizinischen und naturwissenschaftlichen Inhalts. Berlin: Weidmannsche Buchhandlung, 1905, pp. 30-31. 
Papyrus Berolinensis 9770 A. B (Aus einer anatomisch-physiologischen Schrift): ed. Karl Kalbfleisch, in Karl Kalbfleisch und Hermann Schöne (herausgegeben von -), Berliner Klassikertexte, Heft III, Griechische Papyri medizinischen und naturwissenschaftlichen Inhalts. Berlin: Weidmannsche Buchhandlung, 1905, pp. 10-19.

Papyrus Cairensis Cattaui (Chirurgisches Fragment, wahrscheinlich Heliodorus):

ed. Jules Nicole, "Fragment d'un Traité de Chirurgie", Archiv für Papyrusforschung und verwandte Gebiete 4 (1908), pp. 269-271.

Johannes Ilberg, “Kommentar”, ibidem, pp. 271-283.

Papyrus Cairensis Musei 10160: Edgar Johnson Goodspeed, "Greek Papyri from the Cairo Museum together with Papyri of Roman Egypt from American Collections", Decennial Publications of the University of Chicago 5 (1902), pp. 1-78; see pp. 6-7 for papyrus Cairo Museum 10160.

Papyrus Genevensis 111 (»Un questionnaire de chirurgie«): Jules Nicole, "Un questionnaire de chirurgie", Archiv für Papyrusforschung und verwandte Gebiete 2 (1903) pp. 1-3.

Papyrus Golenischtschew; saec. III (Gynaekologische Fragment): Albert Bäckström, "Fragment einer medizinischen Schrift", Archiv für Papyrusforschung und verwandte Gebiete 3 (1906), pp. 158-162.

Papyri graecae Musei Britannici et Musei Berolinensis (De maxilla reponenda. De excrementis. De ponderibus et mensuris): Karl Kalbfleisch, "Papyri Graecae Musei Britannici et Musei Berolinensis", in Ad Scholas quae in hac Universitate Rostochiensi per semestre aestivum inde a D. XV M. Aprilis usque a D. XV M. Augusti A. MDCCCII babebuntur invitant Rector et Concilium. Rostochii: Typis academicis Adlerianis, 1902. pp. 3-14.

De maxilla reponenda: see pp. 3-8; De excrementis: see pp. 8-9 (same as Papyrus Berolinensis 9095 above); De ponderibus et mensuris: see pp. 11-14.

Papyrus Magica: Frederic George Kenyon, Greek Papyri in the British Museum. Catalogue, with texts. London: British Museum, 1893.

Reference is made to pp. $89 \mathrm{ff}$. This is a part of the description of papyrus CXXI described pp. 83-115. At pp. 89-90, Kenyon reproduces, and comments on, the text of 11. 168-185, which contain, according to him (see p. 89, note ad 11. 168$185)$, "A number of recipes for various purposes, not magical". 
Papyrus Oxyrhynchus, nr. 234 (Recepte): Bernard P. Grenfell and Arthurs S. Hunt, The Oxyrhynchus Papyri, Part II. Edited with translations and notes (Egypt Exploration Fund, Graeco-Roman Branch). London: Egypt Exploration Fund, and Kegan Paul, Trench, Trübner \& Co., 1899, pp. 134-136.

Vgl. Alessandro Olivieri, "Papiro medicinale (Pap. di Oxyrhynchus Vol. 2, 1356)”, Bolletino di filologia classica 8 (1901-1902), pp. 229-237.

Vgl. Wilhelm Crönert "Adnotamenta in papyros Musei Britannici graecas maximam partem lexicographica”, Classical Review 17 (1903) pp. 26-27.

$V g l$. Wilhelm Crönert, "Sprachliches zu griechischen Ärzten, eine Untersuchung über den Verfasser des griechischen Papyrus Lond. Nr. 155”, Archiv für Papyrusforschung und verwandte Gebiete 2 (1903), pp. 475-482.

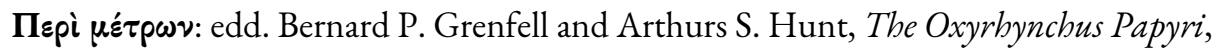
Part IV. Edited with translations and notes (Egypt Exploration Fund, Graeco-Roman Branch). London: Egypt Exploration Fund, and Kegan Paul, Trench, Trübner \& Co., 1904, pp. 116-121. 


\section{Reference Works}

The list of reference works provided at the end of the 1908 Nachtrag (Supplement) provides over 40 titles referenced in a summary form. It is reproduced below with the full references of cited works formatted according to current standard bibliographical practices. For purposes of clarity, full references are introduced by a short reference in boldface, utilizing the last name of the author(s) and the year of publication of the work. The full reference follows in regular typeface.

Listed works range from the classical Elenchus medicorum veterum compiled by Johann Albert Fabricius (1668-1736) and published in 1726 or the Bibliothecae (botanica, chirurgica, anatomica and medicinae practicae) by Albrecht von Haller (17081777) published between 1771 and 1788, to the more recent inventoires and descriptions of Greek medical manuscripts by Georges Costomiris (1849-1902), the revised edition (then in the making) of Paulys Realencyclopädie der classischen Altertumswissenschaft or the recent Hanbuch der Geschichte der Medizin edited by Max Neuburger (1868-1955) and Julius Pagel (1851-1912). The works are mostly in German, with some in French and few in English, besides earlier works in Latin. While they reflect the intellectual tradition of which Diels' catalogues were the heirs, they also integrate the new medical history in the making at that time, with a broad range of disciplines: neurology, ophthalmology, obstetrics, surgery, epidemiology, venereology, and surgery, in addition to pharmacy, botany and materia medica, and also medical education and hospital.

André-Pontier 1900: Léon-Charles André-Pontier, Histoire de la pharmacie. Origines, Moyen Age, Temps Modernes. Paris: Octave Doin, 1900.

Berendes 1891: Julius Berendes, Die Pharmazie bei den alten Culturvölkern. Historisch-kritische Studien, 2 Bände. Halle: Tausch \& Grosse 1891.

Choulant 1841: Ludwig Choulant, Handbuch der Bücherkunde für die ältere Medicin zur Kenntniss der griechischen, lateinischen und arabischen Schriften im ärztlichen Fache und zur bibliographischen Unterscheidung ibrer verschiedenen Ausgaben, Uebersetzungen und Erläuterungen, 2. Auflage. Leipzig: Leopold Voss, 1841.

Choulant 1842: Ludwig Choulant, Bibliotheca medico-historica sive catalogus librorum historicorum de re medica et scientia naturali systematicus. Lipsiae: Gul. Engelmann, 1842.

Costomiris 1889: Georges A. Costomiris, "Etudes sur les écrits inédits des anciens médecins grecs et ceux dont le texte original est perdu, mais qui existent en latin ou en arabe. Première série: Hippocrate, Crateuas, Aelius Promotus, Galien”, Revue des études grecques 2 (1889), pp. 343-383.

Costomiris 1890: Georges A. Costomiris, "Etudes sur les écrits inédits des anciens médecins grecs. Deuxième série: L’Anonyme de Daremberg, Métrodora, Aétius”, Revue des études grecques 3 (1890), pp. 145-179. 
Costomiris 1891: Georges A. Costomiris, "Etudes sur les écrits inédits des anciens médecins grecs. Troisième série: Alexandre (Sophiste et Roi), Timothée, Léon le Sophiste, Théophane Nonnos, les Ephodes”, Revue des études grecques 4 (1891), pp. 97-110.

Costomiris 1892: Georges A. Costomiris, "Etudes sur les écrits inédits des anciens médecins grecs. Quatrième série: Hippiatriques et auteurs du $\mathrm{XI}^{\mathrm{e}}$ siècle: Psellus, Siméon Seth, Damnastès”, Revue des études grecques 5 (1892), pp. 61-72.

Costomiris 1897: Georges A. Costomiris, "Etudes sur les écrits inédits des anciens médecins grecs. Cinquième série: $\mathrm{XIII}^{\mathrm{e}}-\mathrm{XIV}^{\mathrm{e}}$ siècles, Jean Tzetzès, Nicolas Myrepsus, Jean Actuarius”, Revue des études grecques 10 (1897), pp. 405-445.

Espérandieu 1894: Emile Espérandieu, Recueil de cachets d'oculistes romains. Paris: Ernest Leroux, 1893 (not 1894).

Fabricius 1726: Johannes Albertus Fabricius, "Elenchus medicorum veterum, praecipue eorum uorum scripta desiderantur, vel de quibus mentio in priscis monumentis occurrit” in: Idem, Bibliotheca graeca, volumen XIII, quo continetur elenchus medicorum veterum, et notitia collectionum ac scriptorum graecorum junctim editorum, liturgicorumque. Accedunt Anonymi Hoescheliani definitiones, latina versione donatae: Sententiae variorum et secundi philosophi, emendatae ex MS. nuncupationes ecclesiasticae quibus utuntur in suo litterarum commercio Graeci recentiores. Theophili Corydalei expositio rhetorices, $\Xi^{2}$. Hamburgi: Theodorus Christophorus Felginer, 1726, pp. 15-456.

Gurlt 1898: Ernst Julius Gurlt, Geschichte der Chirurgie und hibrer Ausübung. Volkschirurgie, Alterthum, Mittelalter, Renaissance, Erster Band. Berlin: August Hirschwald, 1898.

Haeser 1875: Heinrich Haeser, Lebrbuch der Geschichte der Medicin und der epidemischen Krankheiten. Erster Band: Geschichte der Medicin im Alterthum und Mittelalter, 3. Bearbeitung. Jena: Hermann Dufft, 1875.

Hahn 1897: Lucien Hahn, Essai de bibliograpbie médicale. Etude analytique des principaux répertoires bibliographiques concernant les sciences médicales; de leur utilité dans les recherches scientifiques. Paris: G. Steinheil, 1897.

Hirsch 1893: August Hirsch, Geschichte der medicinischen Wissenschaften in Deutschland (Geschichte der Wissenschaften in Deutschland, Neuere Zeit. 22). München und Leipzig: Druck und Verlag R. Oldenbourg, 1893.

Hirschberg 1899: Julius Hirschberg, Geschichte der Augenheilkunde im Altertbum (Handbuch der gesamten Augenheilkunde, 2. Auflage, 12. Band, Kapitel XXIII. Geschichte der Augenbeilkunde, Erstes Buch). Berlin: Springer, 1899.

Hoffmann 1838-1845: Samuel Friedrich Wilhelm Hoffmann, Lexicon bibliographicum. Sive Index editionum et interpretationum scriptorium graecorum tum sacrorum tum profanorum, zweite umgearbeitete, verbesserte und fortgesetzte Ausgabe, 3 Bände. Leipzig: A. F. Böhme, 1838-1845 (the $2^{\text {nd }}$ edition [1845] referred to here is titled Bibliographisches Lexicon der gesamten Literatur der Griechen). 
Index-Catalogue: Index-Catalogue of the library of the Surgeon-generals Office, United States Army. Authors and Subjects, 16 Bde. Washington: Government Printing Office, 1880-1895; Second Series, 1896 ff.

Kühn 1826-1837: Carolus Gottlob Kühn, Additamenta ad elenchum medicorum veterum, a Io. A. Fabricio in biblioth. graec. vol. XIII, p. 17-456 exbibitum, IXXX. Lipsiae: Universitas Literarum Lipsiensis, 1826-1837.

Kühn 1829-1830: Carolus Gottlob Kuehn, Index medicorum oculariorum inter Graecos Romanosque. I-XI. Lipsiae: Universitas Literarum, 1829-1830.

Kühn 1830-1832: Carolus Gottlob Kuehn, Additamenta ad indicem medicorum arabicorum a J. A. Fabricio in bibl. Grec. Vol. XIII. exbibitum. Manip. I-IX. Lipsiae: Universitas Literarum Lipsiensis, 1830-1832.

Magnus 1901: Hugo Magnus, Die Augenbeilkunde der Alten. Breslau: J.U. Kern, 1901.

Meyer 1854 ff.: Ernst H. F. Meyer, Geschichte der Botanik. 4 Bände. Königsberg: Gebrüder Bornträger, 1854-1857.

Neuburger-Pagel 1902: Max Neuburger und Julius Pagel (herausgegeben von -), Handbuch der Geschichte der Medizin, Erster Band. Jena: Gustav Fischer, 1902.

Oehler 1906-1907: Johann Oehler, Epigraphische Beiträge zur Geschichte des Ärztestandes (Jahres-Bericht des k. k. Maximilians -Gymnasiums in Wien. Für das Schuljahr 1906/1907). Wien: Selbstverlag des Gymnasiums, 1907, pp. 3-25.

Pagel 1898: Julius Pagel, Historisch-Medicinische Bibliographie für die Jabre 18751896 (Geschichte der Medizin, Zweite Theile, II). Berlin: S. Karger, 1898.

Pauly 1872: Alphonse Pauly, Bibliographie des sciences médicales. $1^{\text {er }}$ fascicule. Paris: Tross, 1872.

Pauly-Wissowa 1894 ff: Georg Wissowa (herausgegeben von -), Paulys Realencyclopädie der classischen Altertumswissenschaft, Neue Bearbeitung. Stuttgart: J. B. Metzlersche Verlagsbuchhandlung, $1894 \mathrm{ff}$.

Petit 1887: Louis Henri Petit, Essais de bibliographie médicale. Conseils aux étudiants sur les recherches bibliographiques, la manière de faire sa thèse, de ranger sa bibliothèque, etc. Paris: G. Masson, 1887.

Pohl 1905: Rudolfus Pohl, De graecorum medicis publicis (Dissertatio inauguralis Berolinensis). Berolini: Georgius Reimer, 1905.

Puschmann 1889: Theodor Puschmann, Geschichte des medizinischen Unterrichts von den ältesten Zeiten bis zur Gegenwart. Leipzig: Veit \& Comp., 1889.

Reinach 1904: Salomon Reinach, "Medicus", in Charles Daremberg, Edmond Saglio et Edmond Pottier, Dictionnaire des antiquités grecques et romaines d'après les textes et les monuments contenant l'explication des termes qui se rapportent aux moeurs, aux institutions, à la religion, aux arts, aux sciences, au costume, au mobilier, à la guerre, à la marine, aux métiers, aux monnaies, poids et mesures, etc., etc. et en général à la vie publique et privée des anciens. Tome troisième, Deuxième partie (L-M). Paris: Librarie Hachette, 1904, pp. 1669-1700. 
Rosenbaum 1842: Julius Rosenbaum, Additamenta ad Lud. Choulanti bibliothecam medico-bistoricam. Halis Saxonum: Jo. Fr. Lippert, 1842.

Rosenbaum 1847: Julius Rosenbaum, Additamenta ad Lud. Choulanti bibliothecam medico-bistoricam. Specimen secundum. Halis Saxonum: C. A. Schwetschke et filius, 1847.

Rosenbaum 1882: Julius Rosenbaum, Geschichte der Lustseuche im Altertbume, nebst ausfübrlichen Untersuchungen über den Venus- und Palluscultus, Bordelle, Noṽos $\delta \dot{\lambda} \lambda \varepsilon \iota \alpha$ der Skythen, Paederastic und andere geschlechtlichen Ausschweifungen der Alten, als Beiträge zur richtigen Erklärung ibrer Schriften für Aerzte, Philologen und Alterthumsforscher. 3. Abdruck. Halle: H. W. Schmidt, Halle, 1882.

Soury 1899: Jules Soury, Le système nerveux central. Structure et fonctions. Histoire critique des théories et des doctrines. Paris: Georges Carré et C. Naud, 1899.

Sprengel 1846: Kurt Sprengel, Versuch einer pragmatischen Geschichte der Arzneikunde, Vierte Auflage mit Berichtigungen und Zusätzen von Julius Rosenbaum, Erster Band: Aelteste Geschichte der Medicin bis zur empirischen Schule. Leipzig: Gebauersche Buchhandlung, 1846.

Virchow's Jabresbericht 1866-1904: “Geschichte der Medizin und der Krankheiten", in Rudolf Virchow und August Hirsch (herausgegeben von -), Jabresbericht über die Leistungen und Fortschritte in der gesammten Medicin 1-38 (1866-1904).

von Haller 1771-1772: Albertus von Haller, Bibliotheca botanica. Qua scripta ad rem herbariam facientia a rerum initiis recensentur, 2 volumes. Tiguri: apud Orell, Gessner, Fuessli, et Socios, 1771-1772.

von Haller 1774-1775: Albrecht von Haller, Bibliotheca chirurgica qua scripta ad artem chirurgicam facientia a rerum initiis recensentur. Tomus I: Tempora ante Annum MDCCX; Tomus II: Ab Anno MDCCX ad nostra tempora. Bernae: apud Em. Haller, et Basileae: apud Joh. Schweighauser, 1774-1775.

von Haller 1774-1777: Albrecht von Haller, Bibliotheca anatomica qua scripta ad anatomen et physiologiam facentia a rerum initiis recensentur. Tomus I: Ad Annum MDCC; Tomus II: Ab Anno MDCCI ad MDCCLXXVI. Tiguri: apud Orell, Gessner, Fuessli, et Socc., 1774-1777.

von Haller 1776-1788: Albrecht von Haller, Bibliotheca medicinae practicae qua scripta ad partem medicinae practicam facientia a rerum initiis recensentur. Tomus I: Ad Annum MDXXXIII; Tomus II: Ab Anno 1534. ad. A. 1647; Tomus III: Ab Anno 1648. ad A. 1685; Tomus IV: Ab Anno 1686 ad A. 1707. Bernae: apud Em. Haller, et Basileae: apud Joh. Schweighauser, 1776-1788.

von Siebold 1901: Eduard Caspar Jacob von Siebold, Versuch einer Geschichte der Geburtshilfe, Zweite Auflage, Erster Band. Tübingen: Franz Pietzcker, 1901.

Wellmann 1891: Max Wellmann, "Die Medicin bis in die zweite Hälfte des zweiten Jahrhunderts", in Franz Susemihl, Geschichte der griechischen Literatur in der Alexandrinerzeit, Erster Band. Leipzig: B.G. Teubner, 1891, pp. 777-828. 
